\title{
Lusioersily
}

\section{Breast density classification in mammograms: An investigation of encoding techniques in binary-based local patterns}

Bin Rampun, A., Morrow, PJ., Scotney, B., \& Wang, H. (2020). Breast density classification in mammograms: An investigation of encoding techniques in binary-based local patterns. Computers in Biology and Medicine, 122, [103842]. https://doi.org/10.1016/j.compbiomed.2020.103842

Link to publication record in Ulster University Research Portal

\section{Published in:}

Computers in Biology and Medicine

Publication Status:

Published (in print/issue): 31/07/2020

DOI:

10.1016/j.compbiomed.2020.103842

\section{Document Version}

Author Accepted version

\section{General rights}

Copyright for the publications made accessible via Ulster University's Research Portal is retained by the author(s) and / or other copyright owners and it is a condition of accessing these publications that users recognise and abide by the legal requirements associated with these rights.

\section{Take down policy}

The Research Portal is Ulster University's institutional repository that provides access to Ulster's research outputs. Every effort has been made to ensure that content in the Research Portal does not infringe any person's rights, or applicable UK laws. If you discover content in the Research Portal that you believe breaches copyright or violates any law, please contact pure-support@ulster.ac.uk. 


\title{
Breast Density Classification in Mammograms: An Investigation of Encoding Techniques in Binary-based Local Patterns
}

\author{
Andrik Rampun ${ }^{\mathrm{a}, \mathrm{b}, *}$, Philip J. Morrow ${ }^{\mathrm{b}}$, Bryan W. Scotney ${ }^{\mathrm{b}}$, Hui Wang ${ }^{\mathrm{c}}$ \\ ${ }^{a}$ Academic Unit of Radiology, Department of Infection, Immunity and cardiovascular \\ Disease, Sheffield University, S10 2RX, UK \\ ${ }^{b}$ School of Computing, Ulster University, Jordanstown, Northern Ireland, BT37 0QB, \\ $U K$ \\ ${ }^{c}$ School of Computing, Ulster University, Jordanstown, Newtownabbey, Northern Ireland, \\ BT37 0QB, UK
}

\begin{abstract}
We investigate various channel encoding techniques when applied to breast density classification in mammograms specifically local binary, ternary and quinary encoding approaches are considered. Subsequently, we propose a new encoding approach based on a seven-encoding technique yielding a new local pattern operator called a local septenary pattern operator. Experimental results suggest that the proposed local pattern operator is robust and outperforms the other encoding techniques when evaluated on the Mammographic Image Analysis Society (MIAS) and InBreast datasets. The local septenary patterns achieved a maximum classification accuracy of $83.3 \%$ and $80.5 \%$ on the MIAS and InBreast datasets, respectively. The closest comparison achieved by the other local pattern operators is the local quinary operator producing $82.1 \%$ (MIAS) and $80.1 \%$ (InBreast) maximum accuracies, respectively.
\end{abstract}

Keywords: Breast mammography, Breast Density, Local Binary Patterns, Local Ternary Patterns, Local Quinary Patterns, Local Septenary Patterns

\footnotetext{
*Corresponding author

Email address: y.rampun@sheffield.ac.uk (Andrik Rampun)
} 


\section{Introduction}

Breast cancer is the leading cause of death in women. Although younger women can also get breast cancer, women over 50 years old have a much higher chance of being affected contributing to over $90 \%$ of the cases [1]. In 2017, according to the recent breast cancer statistics report by [2], more than 250,000 cases of breast cancer were diagnosed in the United States. Breast Cancer UK revealed that over 11,000 women died in the United Kingdom (UK) in 2016 [3]. Although the causes are not fully understood, several risk factors such as age, family history, overweight/obesity and excessive use of alcohol were found to be the risk contributors of the disease. According to the National Health Services (NHS) in the UK, there are several symptoms of breast cancer such as the appearance of lesions, micro-calcification, breast density and change of size and shape of the breast [4]. Nowadays, mammography is the most common imaging technology used for screening breast cancer to find early symptoms of breast cancer. Other alternative technologies are Ultrasound and Magnetic Resonance Imaging (MRI). In current clinical practice, radiologists have to examine each mammogram of a patient to find any signs of abnormality and doctors can tell whether a tumour is benign or malignant through biopsy tests. Although the overall current clinical methods have significantly improved in the last two decades, there are still some deficiencies such as inconsistency among radiologists, and the fact that it is time-consuming and invasive.

Women with a dense breast are six times more likely to develop cancer mainly because a tumour has a similar appearance to dense tissue, making it difficult to detect in mammograms $[5,6]$. Several studies have indicated that there is a significant correlation between breast density and developing breast cancer $[5,6]$. In most cases, dense breasts contain more glandular and fibrous tissue. In mammograms, breast regions with dense tissue tend to have more brighter pixels with fatty tissue usually represented by darker pixels. During the screening procedure, breast density estimation is visually assessed by radiologists and classified into four groups based on the amount of dense tissue. The Breast Imaging-Reporting and Data System (BI-RADS) fourth edition is one of the guidelines used in breast density assessment and contains four main categories:

1. BI-RADS I (0-25\% dense tissues and mostly fatty)

2. BI-RADS II (26-50\% dense tissues with some scattered density) 


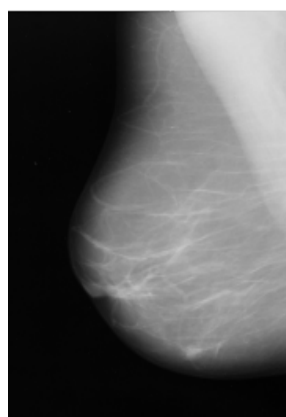

BI-RADS I

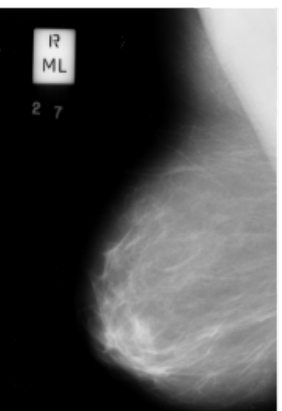

BI-RADS II

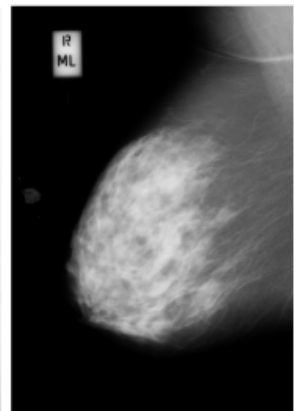

BI-RADS III

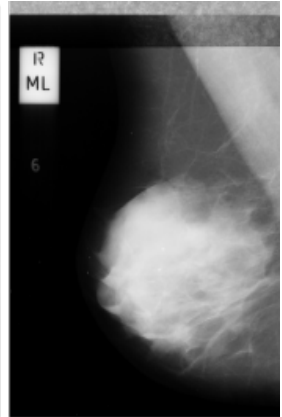

BI-RADS IV

Figure 1: An illustration of breast density according to the BI-RADS guideline fourth edition taken from the the Mammographic Image Analysis Society (MIAS) dataset.

3. BI-RADS III (51-75\% dense tissues and many areas of fibrous and glandular tissue that are evenly distributed)

4. BI-RADS IV (over $75 \%$ dense tissues and breasts have a lot of fibrous and glandular tissue which makes it hard to see a cancer because it can blend in with the normal tissue)

Although manual assessment can be done by radiologists, this task is time-consuming and often suffers from variability between radiologists. For example, radiologists with more experience tend to produce more consistent results compared to less experienced radiologists. Moreover, manual assessment also could lead to false negatives/positives due to fatigue during diagnostic decision making which could influence the final outcome. Figure 1 shows examples of four breasts with their associated BI-RADS classes. In this study we use the fourth edition for simplicity because all datasets are annotated for ground truth based on the BI-RADS fourth edition. However, we are aware that the most recent BI-RADS guidelines is the fifth edition.

\section{Computer-aided diagnosis (CAD) systems}

Computer-aided diagnosis (CAD) systems can assist clinicians regarding efficiency, effectiveness and consistency. CAD systems can assess lesions un-invasively and make predictions as to whether a lesion is benign or malignant. Furthermore, CAD can be used as a 'second reader' to assist clinicians in diagnostic decision making particularly in cases where doctors are not certain about their decision. However, developing a CAD system that can 
replicate radiologists' knowledge requires a significant amount of time and effort. Machine learning is a sub-component of Artificial Intelligence which enables machines to learn and predict a future occurrence of an event. With the use of machine learning and image processing techniques, it is possible to 'teach' computers to learn the appearance/characteristics of breast regions during the training phase and subsequently make a prediction based on the information/knowledge learned.

In breast CAD systems, one of the ways to characterise a breast region in a mammogram is by studying its texture appearance in order to extract meaningful information. In the last decades, many CAD systems for breast imaging have been developed and the majority of them used texture information to investigate the appearance of abnormality such as lesions, dense tissue and micro-calcification clusters. Texture information can be extracted using different mathematical operations such as filter-based, histograms, grey-level distributions, or statistical and probability techniques. Since 2012, the success of deep learning in image classification and segmentation has been overwhelming computer scientists. As a result, hundreds of papers about deep learning for medical image analysis have been published according to the studies by [7] and [8]. Nevertheless, the primary deficiency of deep learning based approaches is the need for a large number of datasets with breast density annotation which can be very challenging in the medical domain. To the best of our knowledge, none of the studies in the literature has attempted using deep learning for four-class breast density classification. The majority of them performed either two-class (low risk (BI-RADS I and II) versus high risk (BI-RADS III and IV)) or three-class (fatty, glandular and dense) classification. On the other hand, many studies have attempted fourclass classification using conventional machine learning in conjunction with texture information.

In this study, we are interested in investigating the effects of various encoding techniques in local pattern extraction by dividing the binary patterns into two (local binary patterns (LBP)), three (local ternary patterns (LTP)) and five (local quinary patterns (LQP)) channels. Subsequently, we propose a local septenary patterns (LSP) operator which divides binary patterns into seven channels. Furthermore, we also study the discriminatory level of local patterns extracted from each channel which can help us to determine more informative texture descriptors. The paper has the following contributions:

1. To the best of our knowledge this is the first study attempting to inves- 
tigate the effects of different encoding techniques covering LBP, LTP and LQP applied to breast density classification.

2. We introduce a variant of LBP (two-encoding technique), LTP (threeencoding technique) and LQP (five-encoding technique) called local septenary patterns (LSP).

3. We introduce several ways of improving local patterns' discriminatory level in the application to breast density classification via a multichannel concatenation approach that enables us to combine local binary patterns from different channels.

4. Whilst threshold values in LTP and LQP need to be set manually by a user, we introduce a simple adaptive way to determine threshold values in LSP based on the intensity distribution of the neighbourhood.

5. Finally we also make a quantitative assessment on each of the channels which can reveal more informative texture descriptors.

Note that our study does not attempt to develop a new CAD system for breast density classification but to investigate the effects on discriminatory levels of local patterns using various encoding techniques.

\section{Breast Anatomy in a Mammogram}

Figure 2 shows breast anatomy in a mediolateral-oblique mammogram (MLO). In many cases, the upper retroglandular region contains fatty tissue and the Corpus Mammae region contains glandular and dense tissue (if the breast is categorised BI-RADS III or IV). Note that usually dense tissue has brighter pixels compared to glandular tissue and these tissues have a similar appearance to the pectoral muscle region. Since many biological activities in the Corpus Mammae region are due to more Lobules and Ducts, dense tissue mostly starts its development in this area.

\section{Literature Review}

Breast density classification in mammograms is one of the most popular topics in breast CAD systems together with mass and micro-calcification clusters classifications. In a majority of the cases, textures have been a popular choice for most authors mainly due to its efficiency and effectiveness. Previously, manual and adaptive thresholding techniques have been used to 


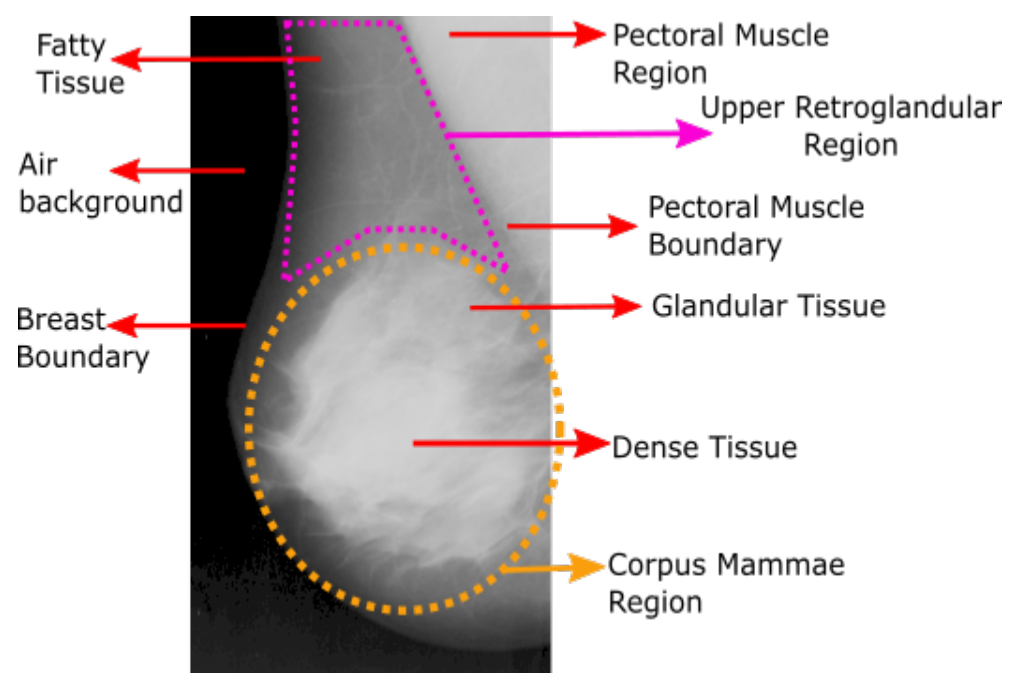

Figure 2: An overview of breast anatomy in a mediolateral-oblique mammogram (MLO). Note that the contrast of the image has been adjusted from the original image for improve visualisation of the breast boundary.

estimate the amount of dense tissue. For example, [9] developed an interactive thresholding software called Cumulus to segment regions with dense tissue by manually tuning a grey-level threshold value. Due to the difficulty in getting annotated mammograms, this topic did not get much attention from computer scientists until the MIAS dataset was made available publicly in 1994 [10].

The first- and second-order (e.g., Grey Level Co-occurrence Matrix (GLCM)) statistical features are among the most popular texture descriptors. For example the studies of [11], [12], [13] and [14] all used these texture descriptors by considering different orientations and resolutions. The methods of [11] and [14] first segment the breast into fatty and non-fatty regions followed by feature extraction on each region and future selection to remove uninformative descriptors. Several machine learning algorithms were employed by [11] and reported an accuracy of $77 \%, 72 \%$ and $86 \%$ for $k$-Nearest Neighbours, C4.5 and Bayesian Network classifiers, respectively. [14] reported an accuracy over $91 \%$ using rough fuzzy approaches with the same feature extraction techniques used by [11]. [13] selected a set of first- and second-order statistical features using a combination of several feature selection techniques and reported a maximum accuracy of $79.2 \%$. [12] extracted features from the 
Spatial Grey Level Dependency matrix (a variant of GLCM) as dense tissue descriptors and employed a combined classifier paradigm based on feedforward Artificial Neural Network (ANN) models to achieve just over $71 \%$ classification accuracy.

Morphology-based descriptors also have been widely investigated to characterise the shape appearance of fatty and non-fatty regions. In many cases, they are used as a set of descriptors with first- and second-order statistical features. For example, [11] and [14] extracted a set of morphology features such as the area, mean, standard deviation and kurtosis using a moment based histogram technique from the segmented fatty and non-fatty regions. The fractal-based feature extraction technique based on a set of threshold values is often used to measure the ratio between the number of low and high grey-levels within a region of interest (e.g., within a $5 \times 5$ neighborhood). For example, [15] divided breast regions by recursively splitting it into quadrants depending on the intensity information that is contained in each region. Subsequently, each subregion is evaluated until the decision function determines that the region does not need to be split, thus obtaining regions with similar properties of tissue. They reported an accuracy of $93 \%$ based on two-class classification (low versus high risk). [16] used fractal analysis to characterise parenchymal patterns in digital mammograms for risk assessment of developing breast cancer, and reported an area under the curve value of 0.86 .

Bag-Of-Words features such as Textons and Scale Invariant Feature Transform (SIFT) have also been studied in the last decade. [17] compared the performance of Textons (extracted from the original images instead of the filtered images) and SIFT features for four-class density classification and used probabilistic Latent Semantic Analysis (pLSA) to obtain a meaningful set of features generating a compact tissue representation of each density class. They reported over $91 \%$ and $88 \%$ accuracies achieved by Textons and SIFT approaches, respectively. In a similar study, [18] compared the performance of various methods for mammographic dense tissue pattern modelling such as the Local Grey-Level appearance (LGA), Textons, LBP and Basic Image Features (BIF). In the Textons approach, [18] used various filter sizes and orientations to extract texture information from filtered images instead of directly from the original images and various parameter values (e.g., window size and the number of neighbours) were tested to extract LGA, LBP, and BIF features. For the four-class BI-RADS classification the authors reported accuracies $75 \%, 72 \%, 59 \%$ and $70 \%$ for Textons, LGA, LBP and BIF, re- 
spectively. [19] implemented a Textons approach based on the Maximum Response 8 (MR8) filter bank. The $\chi^{2}$ distribution was used to compare each of the resulting histograms from the testing set with all the learned histogram models from the training set and reported $75.5 \%$ accuracy.

In recent studies, [20, 21] showed that both LTP (three-encoding approach) and LQP (five-encoding approach) operators can achieve $77 \%$ to $85 \%$ accuracy using a multiscale approach with different neighbourhood topologies and different number of dominant patterns. Instead of extracting texture features from the entire breast region, [20, 21] showed that extracting texture information only from the Corpus Mammea reduces the number of overlapping features and hence produces more distinctive features. The authors showed that this approach was at least $7 \%$ better in terms of classification accuracy compared to classifying breast density by extracting features from the entire breast region. [22] modelled the distribution of breast density based on the multi-scale distribution of dark ellipses (representing fatty tissue) and bright ellipses representing fibroglandular and dense tissues. Subsequently, morphological features such as distances, sizes and diameters were extracted to estimate the amount of dense tissue in the breast. Preliminary results show that their method achieved accuracy between $67 \%$ to $72 \%$ based on various classifiers. Later, [23] compared the performance between Elliptical LBP (ELBP), uniform ELBP and Mean-Elliptical LBP (M-ELBP) for threeclass classification (fatty, glandular and dense) and reported classification accuracies of $75 \%, 74 \%$ and $80 \%$, respectively.

Despite a large number of methods having been developed to address the breast density classification problem in mammograms, only a few studies have achieved accuracies above $80 \%$ and the majority of the methods produced between $70 \%$ to $79 \%$. Moreover, most studies were based on two-class classification (low risk (BI-RADS I and II) versus high risk (BI-RADS III and IV)) or three-class classification (fatty, fatty-glandular and dense-glandular) instead of four-class classification. This might be due to challenging issues such as complex and overlapping tissue appearance as well as ambiguous texture patterns which make it difficult to separate BI-RADS classes based on texture.

In the last a few years, Convolutional Neural Network (CNN) and Deep Residual Learning (ResNet) are among the most popular deep learning techniques which have been used to address classification and segmentation problems in clinical image data $[8,7]$. Many current studies have claimed that deep learning based methods produced superior results achieving accuracy 
similar to human performances $[8,7,24,25]$. In breast density classification, the majority of the studies used deep learning based methods to address either two-class (scattered and heterogeneously density) or three-class classification problems (fatty, glandular and dense tissue). [26] developed a CNN model to distinguish between scattered density and heterogeneously dense tissues using over 22,000 images. [27] used deep learning networks to classify dense and non-dense samples for the purpose of dense tissue segmentation. [28] used unsupervised deep learning to segment dense tissue in mammograms and estimate the risk of developing breast cancer based on the of segmentation result. Recently, [29] reported that their deep learning classification results are correlated well with BI-RADS density assessments by radiologists and comparable with a state of the art algorithm, Laboratory for Individualized Breast Radiodensity Assessment (LIBRA).

Although deep learning based methods have shown promising results, breast density classification based on the BI-RADS four-class assessment guideline (fourth edition) remains a challenging task. This might be due to a lack of annotated data and the complexity of the task itself. According to the recent study of [8], the main challenge of employing a deep learning network is the requirement of a large dataset and annotations from experts. In addition, deep learning suffers from a lack of direct human interpretability because deep learning features rely on filter responses solicited from a large amount of training data whereas hand-crafted features such as those extracted from local patterns provide transparent information, which are more interpretable to clinicians and researchers. Furthermore, all datasets available publicly suffer from an imbalance in the number of images for each class. For example, in the MIAS [10] and InBreast [30] datasets the number of BI-RADS IV cases is less than $10 \%$ of the total number of cases. As a result, deep learning networks may suffer from an imbalanced classification problem.

\section{Methodology}

This section explains the technical details of our study covering steps involved in the pre-processing, feature extraction and the classification phase. Figure 3 shows a general overview of the workflow in our experiments.

Firstly, we performed breast segmentation to separate the breast boundary and pectoral muscle using the method proposed by [31]. This ensures that only local patterns within the breast region will be extracted. Follow- 


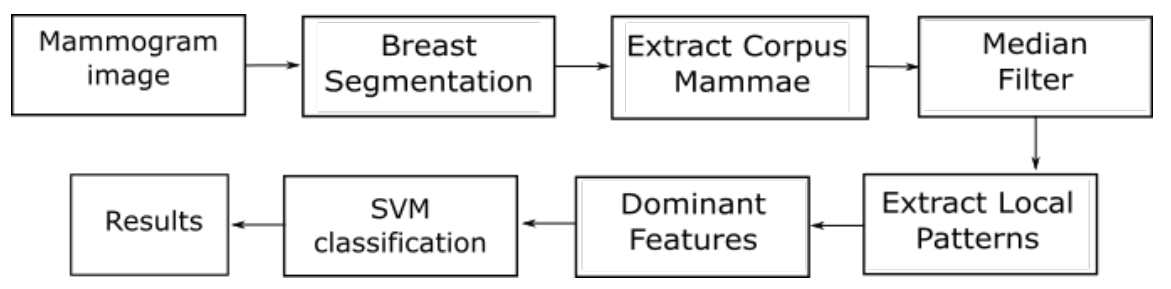

Figure 3: An overview of the work flow involved in our experiments.

ing our previous studies [20,21], we extract the Corpus Mammae region of the breast to reduce the risk of overlapping local patterns across BI-RADS classes. Subsequently, we used a median filter around $3 \times 3$ neighbouring pixels for noise reduction. We extract local patterns at different channels using the LBP, LTP, LQP and LSP operators within the estimated Corpus Mammae region. We select a set of dominant patterns to remove uninformative features and employ the Support Vector Machine (SVM) as a classification approach after a zero-mean normalisation.

\subsection{Pre-processing}

To segment the breast region, we used our previously developed method [31] to estimate the breast and pectoral muscle boundaries. Based on the estimated boundaries we created a breast mask and performed a pixel wise multiplication with the original image resulting in an image with only the breast region. The method [31] employs a region-based Active Contour to estimate the breast boundary and restricted contour growing with edge information for the pectoral muscle boundary estimation. Figure 4 shows an example of an extracted Corpus Mammae region of interest $\left(R O I_{c m}\right)$ and a few examples of estimated breast and pectoral muscle boundaries.

The left-most image in Figure 4 shows the estimated $R O I_{c m}$ area (amber square box). To extract $R O I_{c m}$, we find the height $\left(B_{h}\right)$ and the width of the breast $\left(B_{w}\right) . B_{h}$ is then relocated to the middle of $B_{w}$ to get the intersection point. The width and height of the square area of the $R O I_{c m}$ (amber line Figure 4) can be computed as $B_{w} \times B_{w}$ with the centre located at the intersection point between the $B_{h}$ and $B_{w}$ lines. $B_{h}$ is the height of the breast, which is the longest perpendicular distance between the $x$-axis and the breast boundary. Note that the size of $R O I_{c m}$ varies depending on the width of the breast and local patterns are only extracted within $R O I_{c m}$ instead of from the whole breast region as the majority of the studies in the literature have 


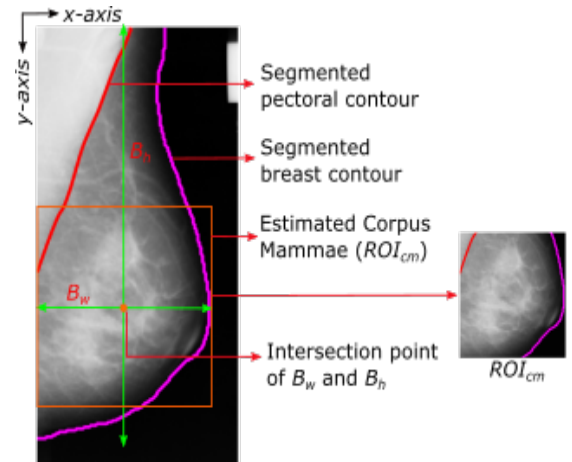

Example of extracted Corpus Mammae

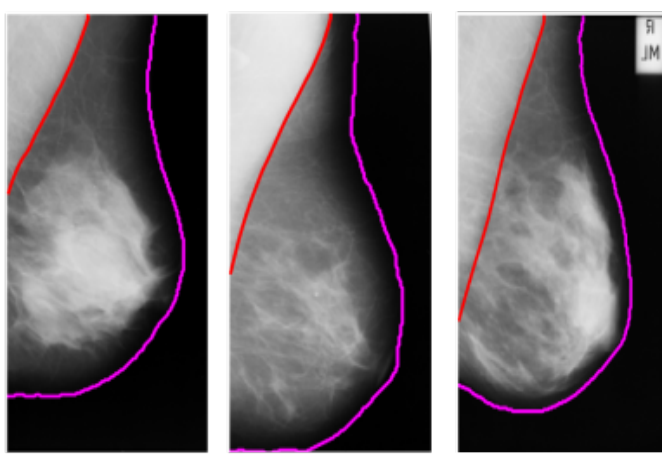

Examples of breast segmentation

Figure 4: Left: an example of extracted Corpus Mammae. Right: examples of breast segmentations.

done.

\subsection{Feature Extraction}

This section explains the original LBP operator [32] and its extensions which are the LTP and LQP operators introduced in [33] and [34], respectively. Subsequently, we propose a LSP operator and explain the different channels in each of the operators. Note that there are several other variants of the LBP operator such as ELBP [35], M-ELBP [36], Median Robust Extended Local Binary Pattern (MRELBP) [37], spatio-temporal LBP (STLBP) [38] and completed LBP (CLBP) [39]. However, they are outside the scope of our study because these operators are based on the LBP's two-channel encoding technique (nevertheless we consider this as part of our future work) whereas the scope of our study is to investigate the effects of local patterns on accuracy when various different different channel encoding techniques are employed.

All these operators use three different mapping tables namely uniform pattern ('u2'), rotation invariant pattern ('ri') and both uniform and rotation invariant patterns ('riu2') to extract uniform, rotation invariant and rotation invariant uniform patterns, respectively. In our study, we investigate the 'riu2' patterns (a combination of ' $\mathrm{u} 2$ ' and 'ri' patterns) as they provide more discriminant features based on our previous studies $[20,21]$ and the study of 
[32]. The value of the LBP code of the pixel $(i, j)$ is given by:

$$
L B P_{(P, R)}(i, j)=\sum_{p=0}^{(P-1)} s\left(g_{p}-g_{c}\right) 2^{p}
$$

where $R$ and $P$ are the radius of the circle that forms the neighbourhood of the operator and the number of pixels in the neighbourhood, respectively. The grey level value of the centre pixel is denoted $g_{c}$, and $g_{p}$ is the grey level value of the $p^{t h}$ neighbour. The LBP operator thresholds the neighbouring pixels using a two-value encoding system as shown in Equation 2.

$$
s(x)= \begin{cases}1, & x \geq 0 \\ 0, & \text { otherwise }\end{cases}
$$

Later, [33] introduced a three-value encoding technique (LTP operator) which thresholds the neighbouring pixels based on a constant threshold set by the user $\left(\tau_{1}\right)$ as shown in Equation 3. Once the LTP code image is generated, it can be separated into two binary patterns from its positive and negative channels. Therefore, it encodes an image into three channels but producing two binary patterns.

$$
s(x)= \begin{cases}1, & x>g_{c}+\tau_{1} \\ 0, & g_{c}-\tau_{1}<x<g_{c}+\tau_{1} \\ -1, & x<g_{c}+\tau_{1}\end{cases}
$$

In [34] the LQP operator was introduced, which thresholds the neighbouring pixels using a five-value encoding technique (see Equation 4) based on two constant thresholds $\tau_{1}$ and $\tau_{2}$. Subsequently, the LQP code image is split into four binary patterns by considering its positives, zero and negative components. Therefore, the LQP operator encodes an image into five channels but results four binary patterns (from two positive channels $(s(1)$ and $s(2))$, zero channel $(s(0))$ and combined negative channels $(s(-1) \cap s(-2)))$.

$$
s(x)= \begin{cases}2, & x \geq g_{c}+\tau_{2} \\ 1, & g_{c}+\tau_{1} \leq x<g_{c}+\tau_{2} \\ 0, & g_{c}-\tau_{1} \leq x<g_{c}+\tau_{1} \\ -1, & g_{c}-\tau_{2} \leq x<g_{c}-\tau_{1} \\ -2, & \text { otherwise }\end{cases}
$$




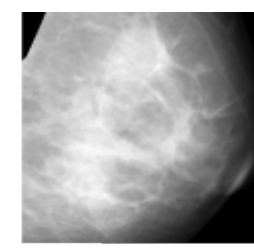

$R O I_{\mathrm{cm}}$

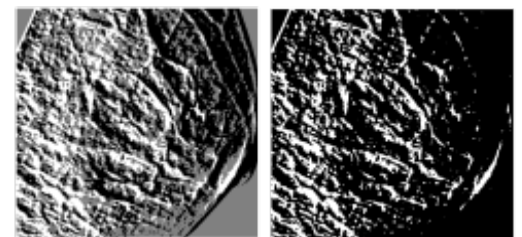

LTP code image LTP Channel 1

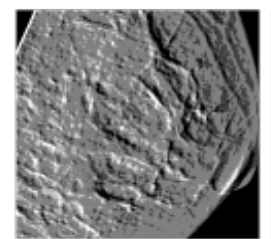

LQP code image

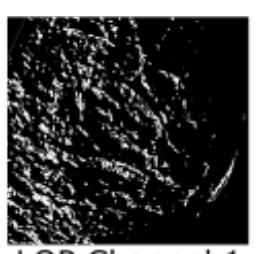

LQP Channel 1
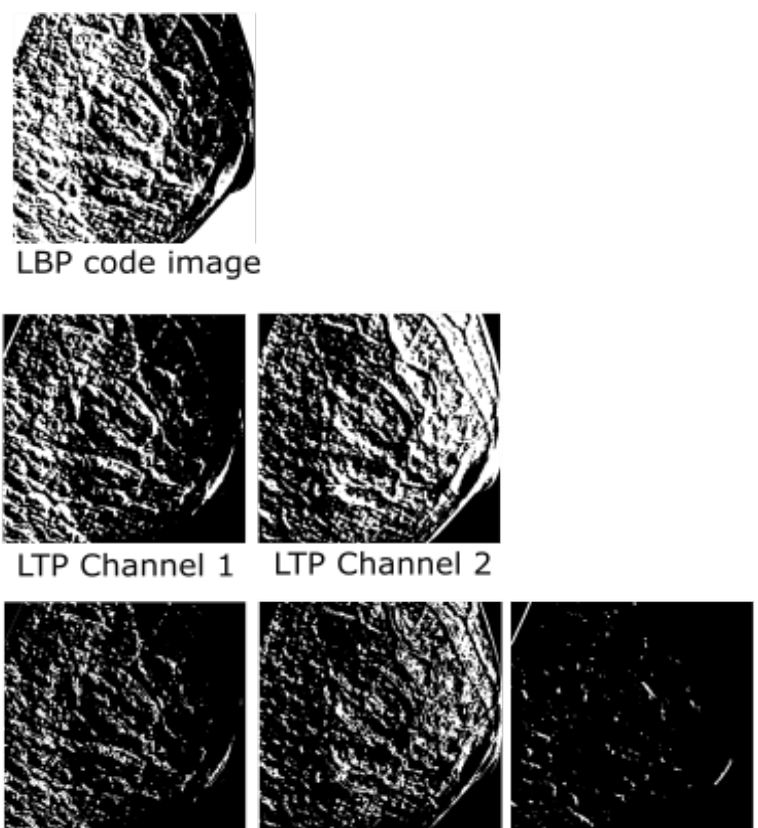

LQP Channel 2

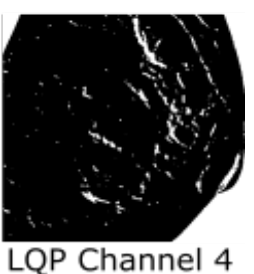

Figure 5: Examples of LBP, LTP and LQP code images and local pattern images from different channels.

We took a similar approach by thresholding the neighbouring pixels using a seven-value encoding technique (hence the name is septenary) based on three threshold values $\left(\tau_{1}, \tau_{2}\right.$ and $\left.\tau_{3}\right)$. The value of the LSP code of the pixel 
$(i, j)$ is given by:

$$
L S P_{(P, R)}^{\text {pattern }}(i, j)=\sum_{p=0}^{(P-1)} s_{\text {pattern }}\left(g_{p}\right) 2^{p}
$$

where pattern $\in\{1,2,3,4,5,6\}$ represents six binary patterns by considering its upper-positive, middle-positive, lower-positive, upper-negative, middlenegative and lower-negative components denoted as $s(3), s(2), s(1), s(-1)$, $s(-2)$ and $s(-3)$, respectively, as shown in Equation 6

$$
s(x)= \begin{cases}3, & x \geq g_{c}+\tau_{3} \\ 2, & g_{c}+\tau_{2} \leq x<g_{c}+\tau_{3} \\ 1, & g_{c}+\tau_{1} \leq x<g_{c}+\tau_{2} \\ 0, & g_{c}-\tau_{1} \leq x<g_{c}+\tau_{1} \\ -1, & g_{c}-\tau_{2} \leq x<g_{c}-\tau_{1} \\ -2, & g_{c}-\tau_{3} \leq x<g_{c}-\tau_{2} \\ -3, & \text { otherwise }\end{cases}
$$

Note that LSP encodes an image into seven channels but results in six binary patterns. The LSP code image is split into six binary patterns using the following conditions

$$
\begin{aligned}
& s_{1}(x)= \begin{cases}1, & \text { if } s(3)=3 \\
0, & \text { otherwise }\end{cases} \\
& s_{2}(x)= \begin{cases}1, & \text { if } s(x)=2 \\
0, & \text { otherwise }\end{cases} \\
& s_{3}(x)= \begin{cases}1, & \text { if } s(x)=1 \\
0, & \text { otherwise }\end{cases} \\
& s_{4}(x)= \begin{cases}1, & \text { if } s(x)=-1 \\
0, & \text { otherwise }\end{cases} \\
& s_{5}(x)= \begin{cases}1, & \text { if } s(x)=-2 \\
0, & \text { otherwise }\end{cases}
\end{aligned}
$$




$$
s_{6}(x)= \begin{cases}1, & \text { if } s(x)=-3 \\ 0, & \text { otherwise }\end{cases}
$$

Figure 6 shows an example of a LSP code image extracted from $R O I_{c m}$ in Figure 5 and its local patterns from different channels. Using the encoding technique in Equation 6, we can generate the LSP code image of $R O I_{\mathrm{cm}}$. The LSP code image is split into six binary images which represent local patterns in six channels. Unlike LTP and LQP where the user has to manually determine threshold values, we introduce an automatic approach by computing the number of neighbours with grey level value $\leq 25^{\text {th }}$ percentile of the entire neighbourhood, number of neighbours with grey level value between $25^{\text {th }}$ and $75^{t h}$ percentile of the entire neighbourhood and number of neighbours with grey level value $\geq 75^{\text {th }}$ percentile. Subsequently, we sort the values in ascending order for $\tau_{1}, \tau_{2}$ and $\tau_{3}$ values.

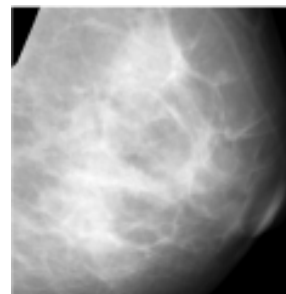

$R O I_{C m}$

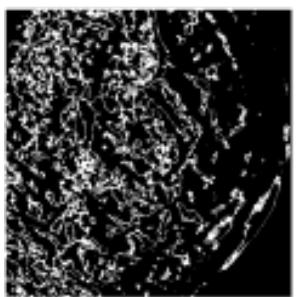

LSP Channel 3

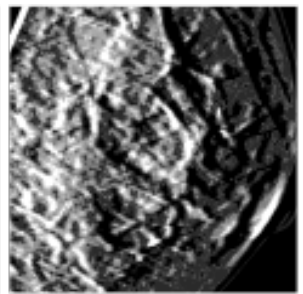

LSP code image

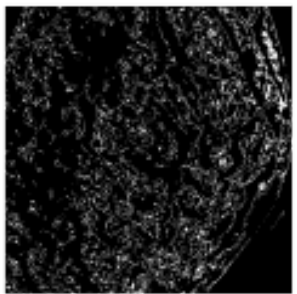

LSP Channel 4

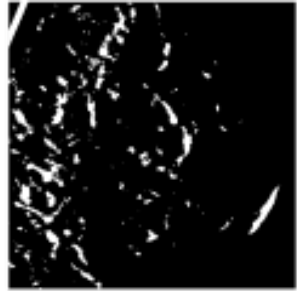

LSP Channel 1

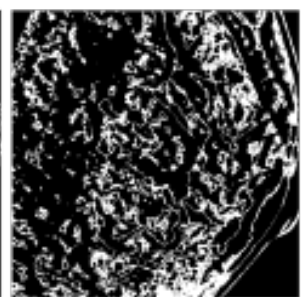

LSP Channel 5

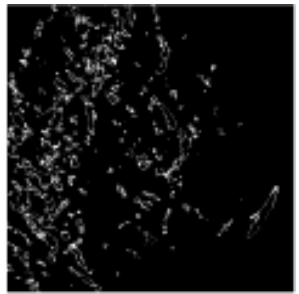

LSP Channel 2

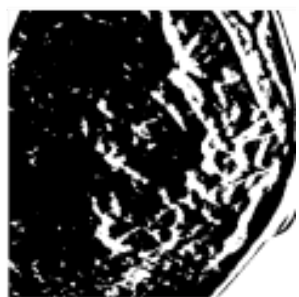

LSP Channel 6

Figure 6: Examples of LSP code image and its local pattern images from different channels.

The LBP, LTP, LQP and LSP are similar in terms of architecture as each is defined using a circle centred on each pixel and the number of neighbours. The main difference is the LSP thresholds the neighbouring pixels into seven $(3,2,1,0,-1,-2$ and -3$)$ values. In contrast, the LBP, LTP and LQP encode an image into two (1 and 0$)$, three (1,0 and -1), and five $(2,1,0,-1$ and -2$)$ values. Figure 7 shows an example of the feature extraction process. The final 
histogram is a concatenation of six histograms computed from each binary pattern (binary image, e.g., LSP code 1) generated based on the conditions in equations (7) to (12). The process of capturing local patterns of $R O I_{c m}$ is also the same for the other operators such as the LBP, LTP and LQP, except less number of channels (resulting in a shorter concatenated histogram).

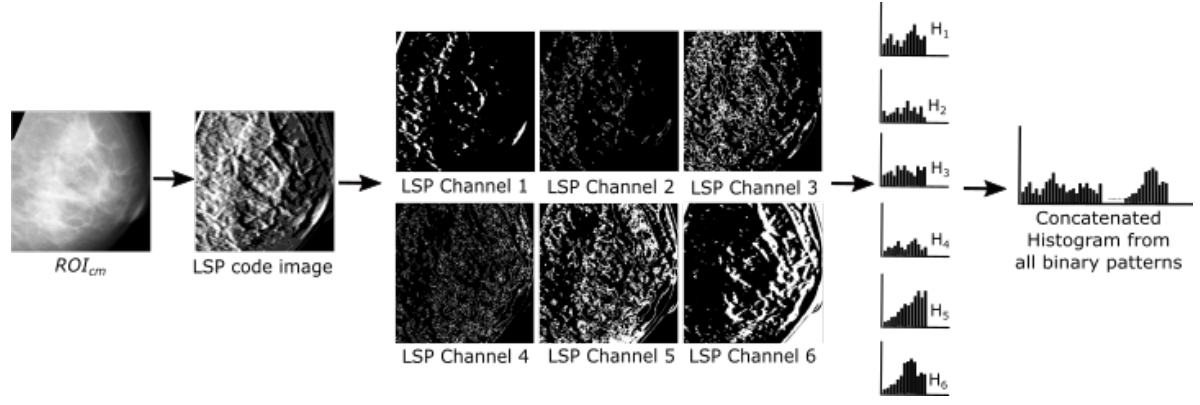

Figure 7: Summary of feature extraction phase using the LSP operator. Note that the binary pattern from each channel is computed from the LSP code image and a histogram image is computed for each channel. Finally, all histograms are concatenated and treated as a feature vector to represent the local pattern of $R O I_{c m}$.

\subsection{Dominant Patterns}

Following our previous studies in $[21,20]$, we selected dominant patterns to reduce feature dimension, hence simplifying the prediction model. Dominant patterns are patterns that occur frequently in the training set [40]. Let $I_{1}, I_{2} \ldots I_{j}$ be images in the training set. We compute the histogram feature $\left(H_{I_{j}}^{L S P}\right)$ for each training image and perform a bin-wise summation for all the histograms to find the pattern's distribution from the training set. Subsequently, the resulting histogram $\left(H^{L S P}\right)$ is sorted in descending order, and the patterns corresponding to the first $D$ bins are selected using the following equation:

$$
D=\arg \min _{N} \frac{\sum_{i=1}^{N-1} H^{L Q P}(i)}{\sum_{i=1}^{2^{P}} H^{L Q P}(i)}>0.01 \times n
$$

where $N$ and $n$ are the total number of patterns and the threshold chosen by the user, respectively. For example choosing $n=98$ means removing patterns that occur with relative frequency of less than $2 \%$ in $H^{L S P}$. The smaller the value of $n$, the smaller the number of patterns selected. 


\subsection{Classification}

Once the feature extraction is completed, we employed several machine learning algorithms in WEKA [41] to build our classification models. For machine learning with only one parameter (e.g., $k$-NN), the CVParameterSelection technique was employed. In contrast, the GridSearch technique was used to explore two parameters for classifiers with two parameters (e.g. RF and SVM). The CVParameterSelection finds the best setting based on options set by the user by optimising the classification accuracy. The GridSearch tests all possible combination of two parameters and selects the best combinations based on the highest accuracy. To find the best parameters for each classifier, 25 patients $(9,7,5,4$ patients for BI-RADS I, II, III and IV, respectively) were selected from the MIAS dataset and 3-fold cross-validation was used to evaluate the performance for each (or pair) of the tested parameter(s) during the optimisation process. The classifier was trained, and in the testing phase, each unseen $R O I_{c m}$ from the testing set is classified as BI-RADS I, II, III or IV. The classifiers used in this study are presented in Table 1.

Table 1: List of classifiers used in this study. The \# sign represents number

\begin{tabular}{ll} 
Classifiers & Default parameters in WEKA \\
\hline Support Vector Machine $(\mathrm{SVM})$ & Complexity $(C)=1.0$, exponent $=1$ \\
Random Forest $(\mathrm{RF})$ & \# of forests $=100$, depth $=0$ (unlimited) \\
Multilayer Perceptron $(\mathrm{MLP})$ & Learning rate $=0.3$, momentum $=0.2$ \\
$k$-Nearest Neighbours $(k-\mathrm{NN})$ & $k=1$, Euclidean distance
\end{tabular}

\section{Experimental Results}

To test the performance of the method we used the following datasets: (a) the Mammographic Image Analysis Society (MIAS) dataset [10] which consists of 322 mediolateral-oblique (MLO) mammograms of 161 women. Films were taken from the UK National Breast Screening Programme and have been digitised to $50 \mu \mathrm{m} \times 50 \mu \mathrm{m}$ and quantised to 8 bits. The distribution for BI-RADS classes is 60 (BI-RADS I), 105 (BI-RADS II), 129 (BI-RADS III) and 31 (BI-RADS IV), and (b) the InBreast dataset [30] which consists of 206 MLO mammograms from 103 patients. Each image is direct digital acquisition on a-Se imaging plates. The pixel size of all images is $70 \mu \mathrm{m} \times$ 
$70 \mu \mathrm{m}$, with 14-bit contrast resolution. The density distribution for the BIRADS classes is as follow: 69 (BI-RADS I), 74 (BI-RADS II), 49 (BI-RADS III), and 14 (BI-RADS IV). Each image contains BI-RADS information (e.g., BI-RADS class I, II, III or IV) provided by an expert radiologist based on the fourth edition of the BI-RADS system. We ran stratified 5-fold cross validation for 10 times.

Accuracy $(A c c)$ is used to measure the performance of the method, which represents the total number of correctly classified images as a proportion of the total number of images.

\subsection{Optimised Parameters}

Table 2 shows a list of parameter values tested and optimised values for the four classifiers employed in this study. Note that, the parameter optimisation was performed based on 25 patients (each BI-RADS class has six or seven patients) taken from the MIAS dataset. Subsequently, we use these parameter values in the testing phase for both MIAS and InBreast datasets. However, for the MIAS dataset we only use 136 patients (272 mammograms) and we excluded 25 patients (50 mammograms) which were used for parameter optimisation. From now on, all parameter settings for the classifiers employed in this study are based on the best parameter values summarised in Table 2.

Table 2: List of parameter options tested in this study.

\begin{tabular}{|c|c|c|}
\hline Classifiers & Parameter tested & Best parameters \\
\hline SVM & $\begin{array}{l}\text { Kernel }=\text { 'Polynomial', } \\
C=1 \text { to } 10 \\
e=1 \text { to } 5\end{array}$ & $C=5, e=1$ \\
\hline $\mathrm{RF}$ & $\begin{array}{l}\text { Number of forests }(r F)=1 \text { to } 165 \\
\text { depth }(D)=0 \text { to } 10\end{array}$ & $r F=70, D=0$ \\
\hline MLP & $\begin{array}{l}\text { Learning rate }(L R)=0.1 \text { to } 2.0(\text { e.g. } 0.1,0.2 \ldots 2.0) \\
\text { momentum }(M)=0.1 \text { to } 2.0(\text { e.g. } 0.1,0.2 \ldots 2.0)\end{array}$ & $L R=0.1, M=0.5$ \\
\hline$k-\mathrm{NN}$ & $\begin{array}{l}k=1-49(\text { e.g. } 1,3,5, \ldots 49) \\
\text { Euclidean distance }\end{array}$ & $k=5$ \\
\hline
\end{tabular}

\subsection{Quantitative Results}

This section presents classification results for LBP, LTP, LQP and LSP operators based on different classifiers. Since the LTP and LQP require 
threshold values from a user, the parameters $\tau_{1}$ and $\tau_{2}$ were selected based on previous studies $[21,20]$. Therefore the $\tau_{1}$ value for the LTP operator is set to 5 and $\tau_{1}$ and $\tau_{2}$ values for LQP are set to 5 and 12, respectively. Note that the average $A c c$ represents the mean accuracy across different numbers of dominant patterns $(n)$ where the maximum Acc is the highest accuracy achieved for $n$ number of dominant patterns. Choosing $n=93$ means removing patterns that occur with a frequency of less than $7 \%$ in the histogram feature. Numerical values represent performance evaluation based on 272 and 206 images for the MIAS and InBreast datasets, respectively. Note that we have used 50 images (of the original 322 images) from the MIAS dataset for parameter optimisation.

Figure 8 and 9 shows classification results using LBP operator for the MIAS and InBreast dataset, respectively. It can be observed that for the MIAS dataset a maximum accuracy of $73.8 \%$ is achieved by $\operatorname{LBP}(7,16)$ (where $R=7, P=16$, e.g. $\operatorname{LBP}(R, P)$ ) using the SVM classifier at $n=91.5$ (removing local patterns with frequency of less than $8.5 \%$ in the histogram features). The best average accuracy of $69.7 \%$ is achieved when employing $\operatorname{LBP}(7,16)$ with $n=92.1$. For the InBreast dataset a maximum accuracy of $73.8 \%$ is achieved by $\operatorname{LBP}(5,10)$ at $n=96.5$ whereas best average accuracy $(70.2 \%)$ is achieved by $\operatorname{LBP}(9,20)$ using the MLP classifier. Overall, the majority of the classification accuracies fall in a range between $65 \%$ to $70 \%$.

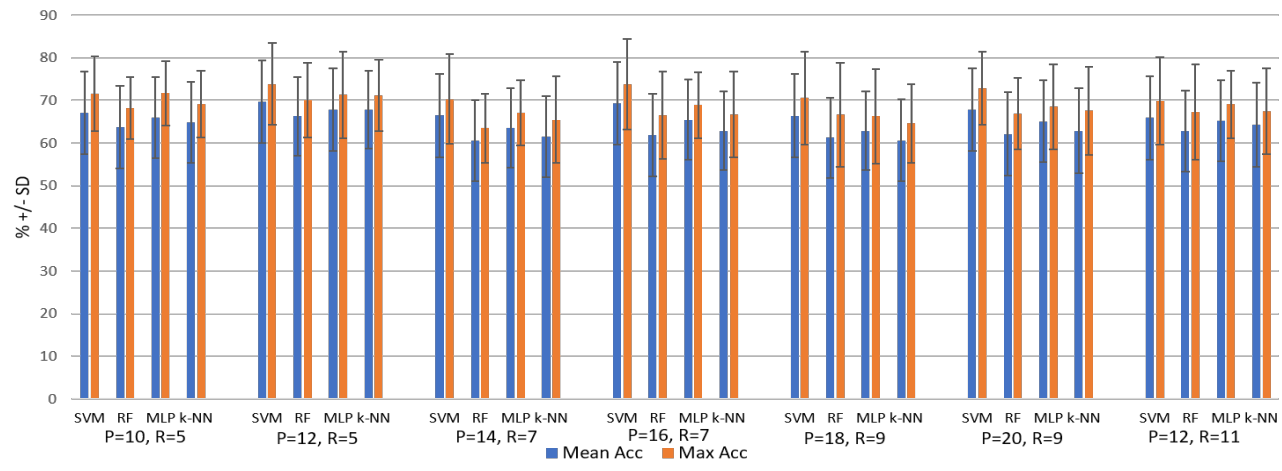

Figure 8: LBP performance on MIAS dataset

Figure 10 and 11 shows quantitative results using the LTP operator for the MIAS and InBreast dataset, respectively. The majority of the average accuracies are in a range between $70 \%$ to $75 \%$ which are higher than the 


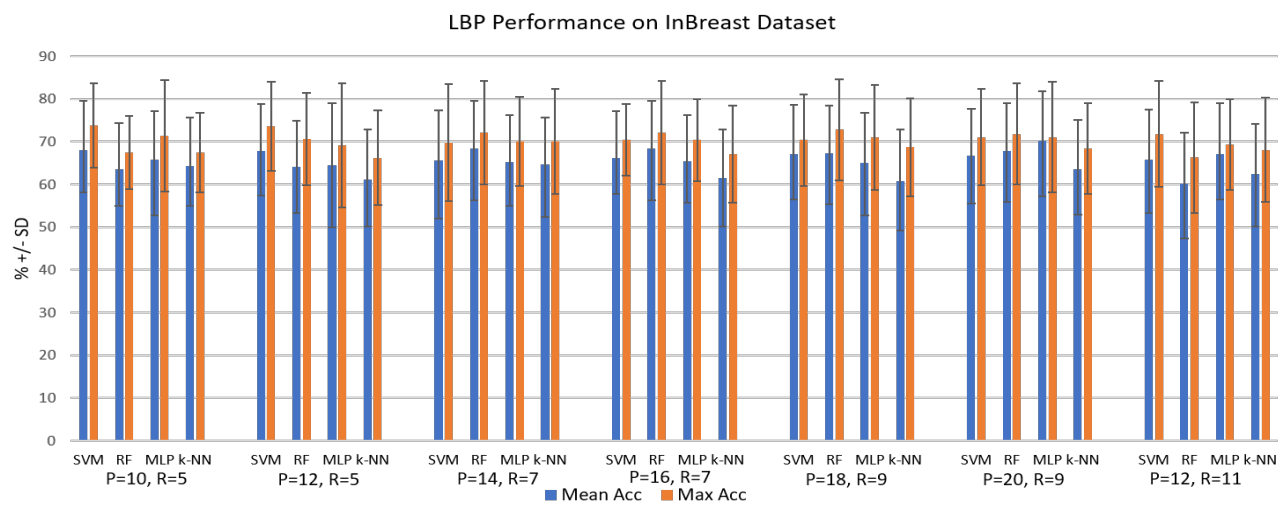

Figure 9: LBP performance on InBreast dataset

ones produced by the LBP operator; for maximum accuracy, many cases are over $75 \%$. Furthermore, it can be observed that the LTP operator outperformed the LBP operator regardless of the $R$ and $P$ values. The highest accuracies are $81 \%$ (average $77.5 \%$ ) and $78.7 \%$ (average $74.3 \%$ ) on the MIAS and InBreast datasets, respectively. They are at least $4 \%$ to $7 \%$ higher than the maximum accuracy produced when extracting local patterns using the LBP operator. The SVM classifier produced the highest classification accuracy by removing $6.5 \%$ of the local patterns when evaluated on the MIAS dataset $(\operatorname{LTP}(7,14)$ and $\operatorname{LTP}(7,16))$ where the MLP classifier $(\operatorname{LTP}(9,18))$ outperformed the other classifiers at $n=93.1 \%$ when tested on the InBreast dataset.

Figure 12 and 13 shows classification results using the LQP operator for the MIAS and InBreast dataset, respectively. Overall, it can be observed that LQP produced better classification results in comparison to LBP and LTP operators. The majority of the maximum accuracies are in a range between $74 \%$ to $79 \%$ which indicates that local patterns extracted by the LQP operator are more discriminant in comparison to the previous two operators. $\operatorname{LQP}(9,18)$ and $\operatorname{LQP}(9,20)$ produced a maximum accuracy of $82.1 \%$ with the best average accuracy of $78.6 \%$ at $n=90$ on the MIAS dataset. However, when evaluated on the InBreast dataset the maximum accuracy of $80.1 \%$ is achieved by $L Q P(9,18)$ at $n=98$ and the best average accuracy is achieved by $\operatorname{LQP}(9,20)$. Moreover, it can also be observed that the SVM classifier produced an average maximum accuracy over $80 \%$ regardless of the values of $P$ and $R$ when evaluated on the MIAS dataset. However, the MLP classifier 


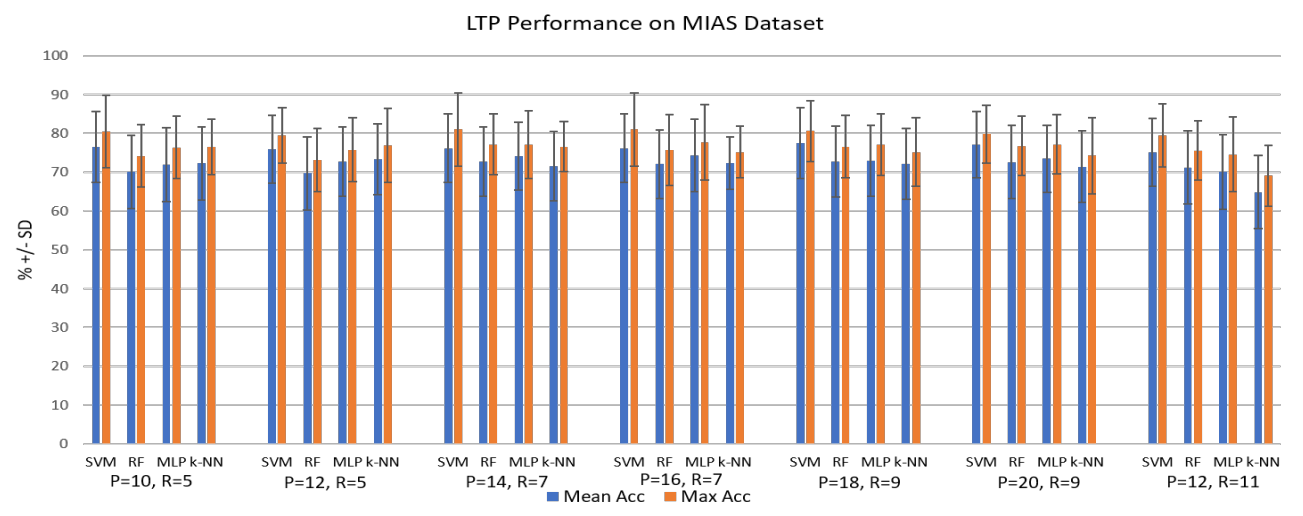

Figure 10: LTP performance on MIAS dataset

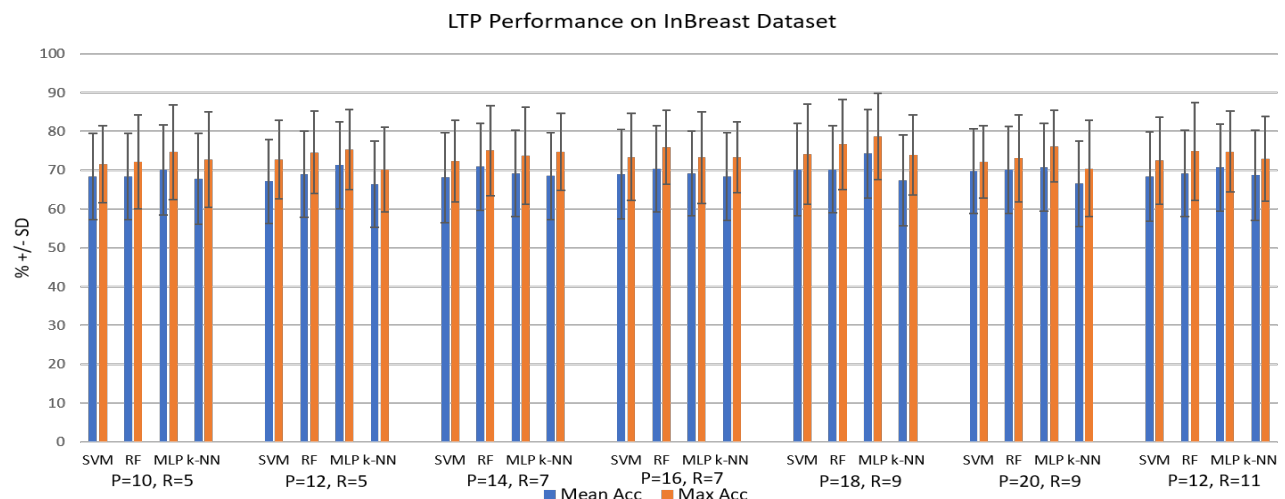

Figure 11: LTP performance on InBreast dataset

tends to produce better results when tested on the InBreast dataset with the best average $A c c=75.5 \%$ which is $1 \%$ better than the SVM classifier.

Table 14 and 15 shows quantitative results using our proposed LSP operator when evaluated on the MIAS and InBreast dataset, respectively. The majority of the classification accuracies fall in the range $75 \%$ to $80 \%$ which is slightly better compared to the results produced by the LQP operator. Experimental results show that the LSP operator produced a maximum accuracy of $83.3 \%$ (which outperforms the LBP (73.8\%), LTP (81\%) and LQP $(82.1 \%)$ operators) on the MIAS dataset. The best average accuracy of $81.6 \%$ is achieved by $\operatorname{LSP}(9,18)$ at $n=99.1$ which is at least $2.8 \%$ higher than the 


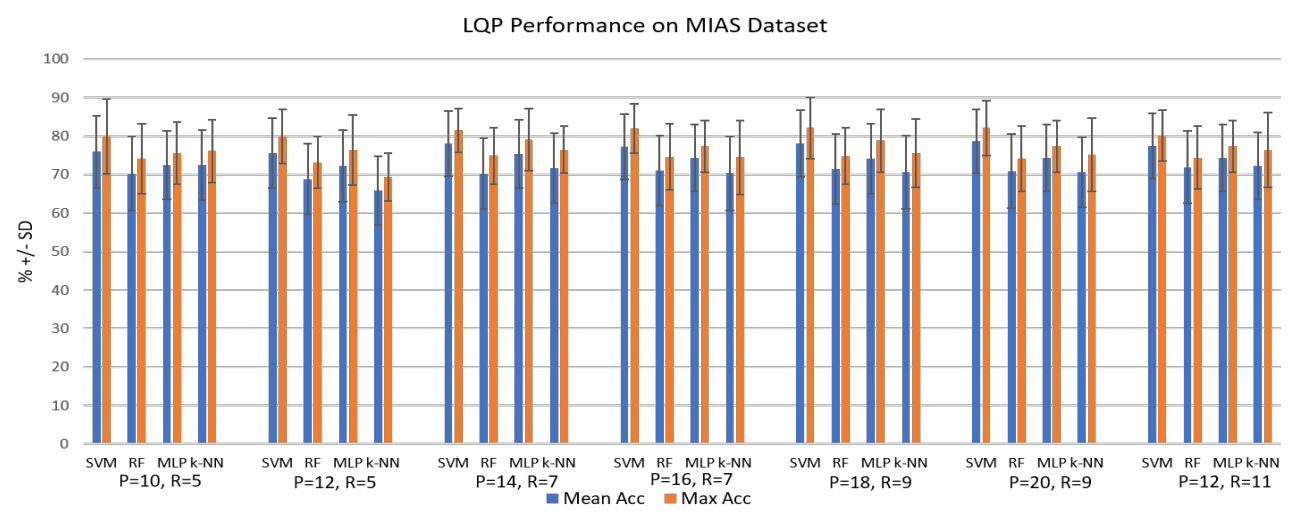

Figure 12: LQP performance on MIAS dataset

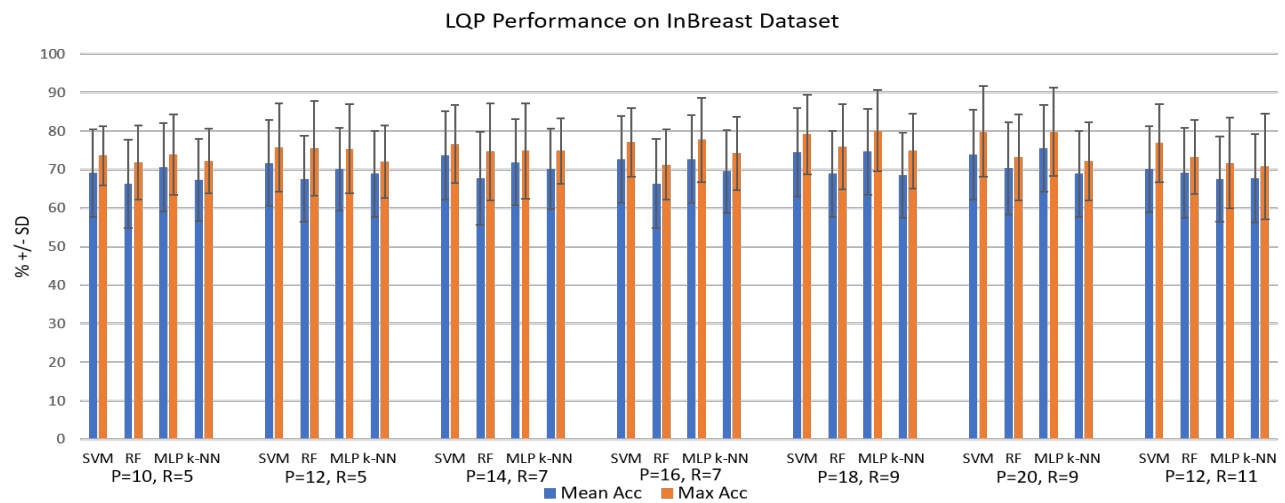

Figure 13: LQP performance on InBreast dataset

best average Acc produced by LQP and LTP. The LSP operator achieved maximum $80.5 \%$ classification accuracy at $n=95.5$ using the SVM classifier whereas the highest accuracy achieved by RF, $k$-NN and MLP are 77.9 $(\operatorname{LSP}(11,22)), 79.7 \%(\operatorname{LSP}(9,20))$ and $79.8(\operatorname{LSP}(7,16))$, respectively. Regarding the best average accuracy across different numbers of dominant patterns $\operatorname{LSP}(9,20)$ produced $77.1 \%$ using the SVM classifier. RF, $k$-NN and MLP produced $70.9 \%, 73.3 \%$ and $73.7 \%$, respectively when $\operatorname{LSP}(9,20)$ is employed. Overall, the LSP operator produced more discriminant local patterns in separating the BI-RADS classes in both MIAS and InBreast datasets. We will discuss this further in the subsequent subsections. 


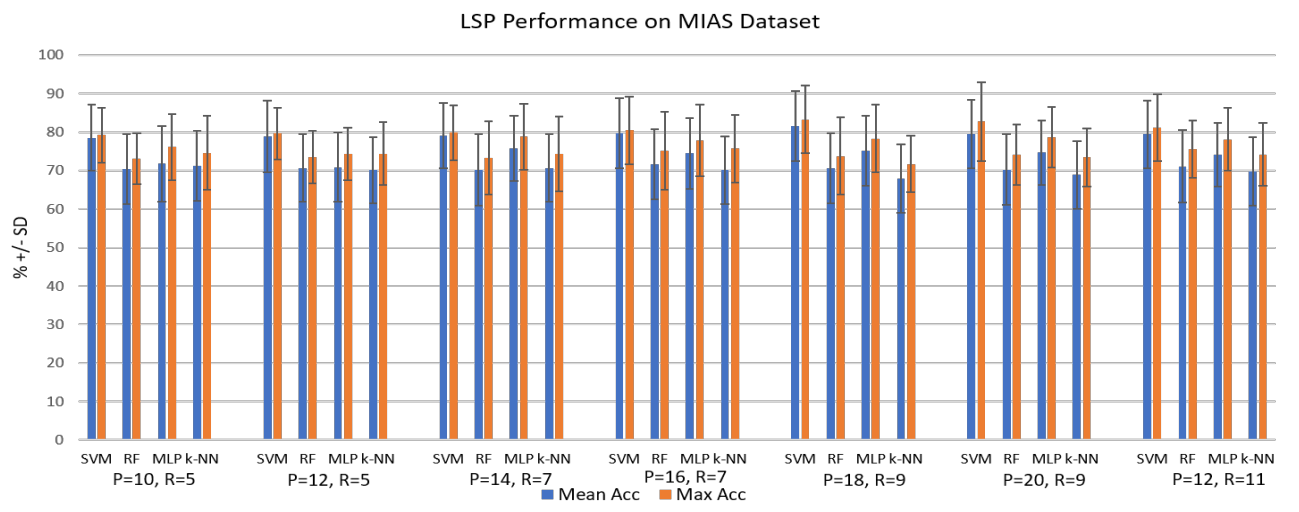

Figure 14: LSP performance on MIAS dataset

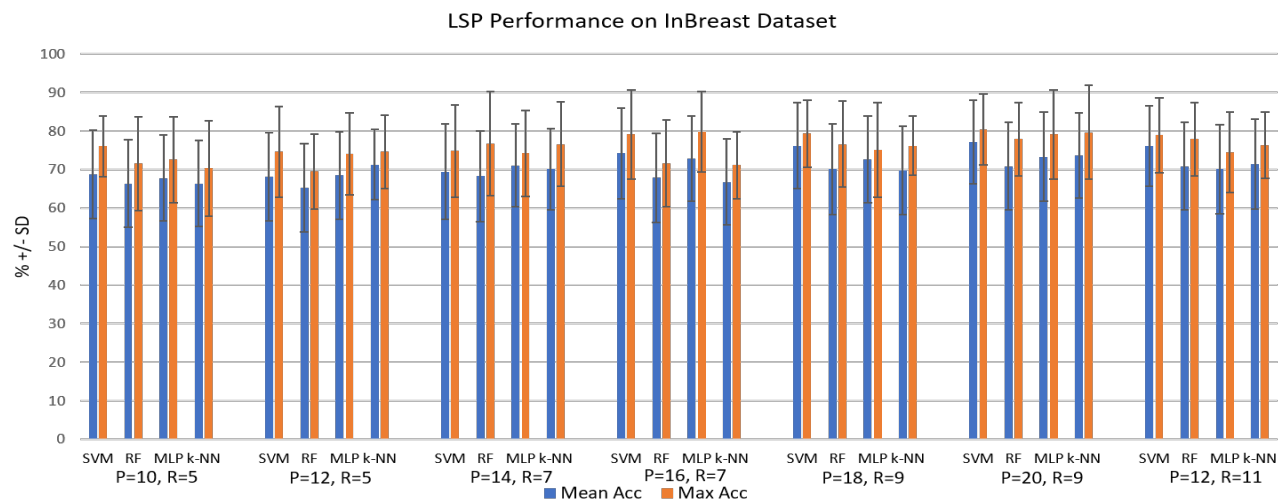

Figure 15: LSP performance on InBreast dataset

\subsection{Maximum and Average Performance Comparison Between Operators}

This section summarises the maximum and average results produced by the operators across different classifiers based on the MIAS and InBreast datasets. For the MIAS dataset, Figures 16 and 17 show performance comparisons between LBP, LTP, LQP and LSP operators using different classification approaches. In terms of maximum accuracy, it can be observed that LSP $(A c c=83.3 \%$ ) outperformed the other texture operators (LBP, LTP and LQP) when using SVM, RF and MLP as classification approaches. However, when the $k$-NN classifier is employed, the maximum classification accuracy is $76.9 \%$ using LTP which is $0.7 \%$ and $1.2 \%$ better than the LQP 
and LSP operators, respectively. Regarding the average accuracies across different numbers of dominant patterns, both the SVM and MLP classifiers produced higher results when using LQP and LSP features. For example, the LSP operator produced $81.6 \%$ and $75.8 \%$ accuracies for SVM and MLP classifiers, respectively compared to $78.6 \%$ (SVM) and $75.4 \%$ (MLP) when classification was performed based on local patterns extracted using the LQP operator. In contrast, classification accuracy is at least $0.9 \%$ better for local patterns using LTP when RF and $k$-NN classifiers are used compared to LQP and LSP. In both Figures 16 and 17, the LBP operator produced the lowest accuracy results regardless to classification approach which clearly shows that a channel encoding technique is necessary to capture more discriminant features and hence improve the classification results.

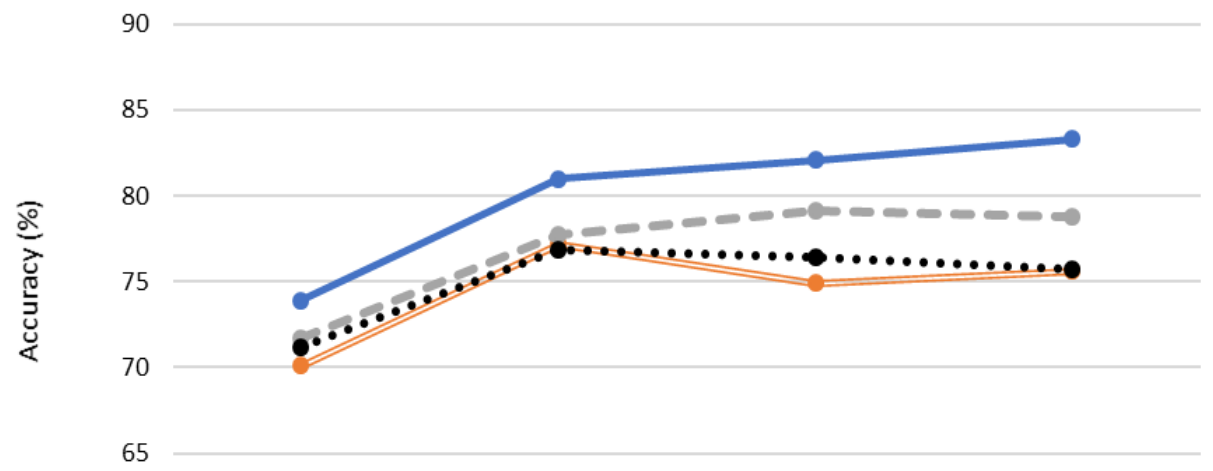

\begin{tabular}{|c|c|c|c|c|}
\hline 00 & LBP & LTP & LQP & LSP \\
\hline- SVM & 73.9 & 81 & 82.1 & 83.3 \\
\hline$\Longrightarrow \mathrm{RF}$ & 70.1 & 77.2 & 74.9 & 75.6 \\
\hline$-\mathrm{C}-\mathrm{MLP}$ & 71.7 & 77.7 & 79.1 & 78.8 \\
\hline$\bullet \bullet \bullet \bullet K N N$ & 71.2 & 76.9 & 76.4 & 75.7 \\
\hline
\end{tabular}

Figure 16: Comparison of maximum accuracies achieved by each operator across different classifiers when evaluated on the MIAS dataset.

For the InBreast dataset, Figures 18 and 19 show performance comparisons between all operators using different classifiers. Regarding maximum accuracy, the LSP operator achieved $A c c=80.5 \%$ using the SVM classifier and outperformed the LBP, LTP and LQP operators by $6.7 \%, 6.4 \%$, and $0.6 \%$, respectively. An improvement also can be observed for the $k$-NN classifier as the LSP operator produced $79.7 \%$ compared to the other operators 


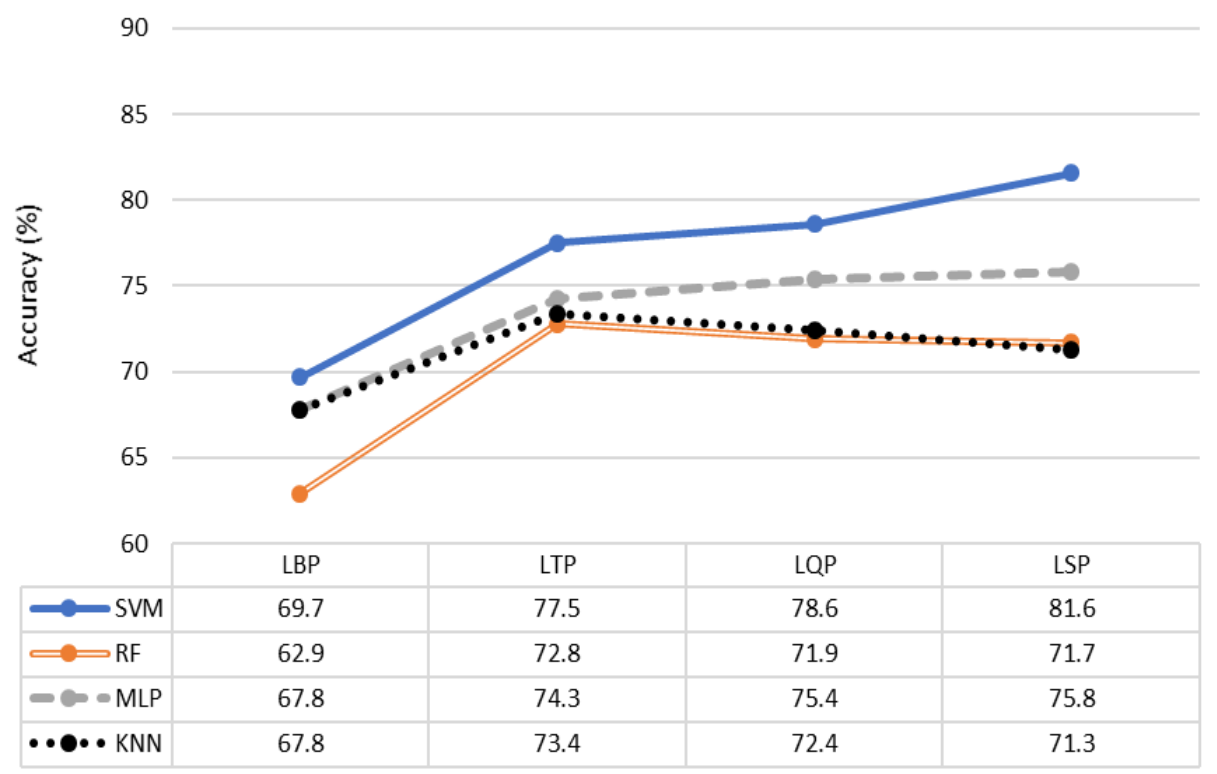

Figure 17: Comparison of average accuracies across different numbers of dominant patterns achieved by each operator across different classifiers when evaluated on the MIAS dataset.

which produced Acc $<75 \%$. For the RF classifier, the LSP operator produced $77.9 \%$, at least $1.2 \%$ higher compared to the other operators. The LQP operator produced a maximum accuracy of $80.1 \%$ which is $0.3 \%$ higher than the LSP operator when using the MLP classifier. Once again it can be observed that other operators always outperform the classification results of the LBP operator regardless of classifiers. In terms of average accuracies, the LSP operator once again outperforms the other operators when the SVM classifier is employed with the best average accuracy of $77.1 \%$. Similar trends can be seen when using the $k$-NN classifier where the LSP features produced the best average accuracy of $73.8 \%$ compared to $64.6 \%, 68.7 \%$ and $70.2 \%$ produced by the LBP, LTP and LQP features, respectively. The LSP and LTP operators produced similar results of $70.9 \%$ when the RF classifier is employed which is $0.6 \%$ higher than the LQP operator. The LSP operator produced the best average accuracy of $73.3 \%$ using the MLP classifier which is slightly lower compared to LQP and LTP operators with $75.5 \%$ and $74.3 \%$ accuracy, respectively. Once again experimental results suggest that the LSP operator extracts more discriminant local features and performs better when the SVM classifier is employed. 


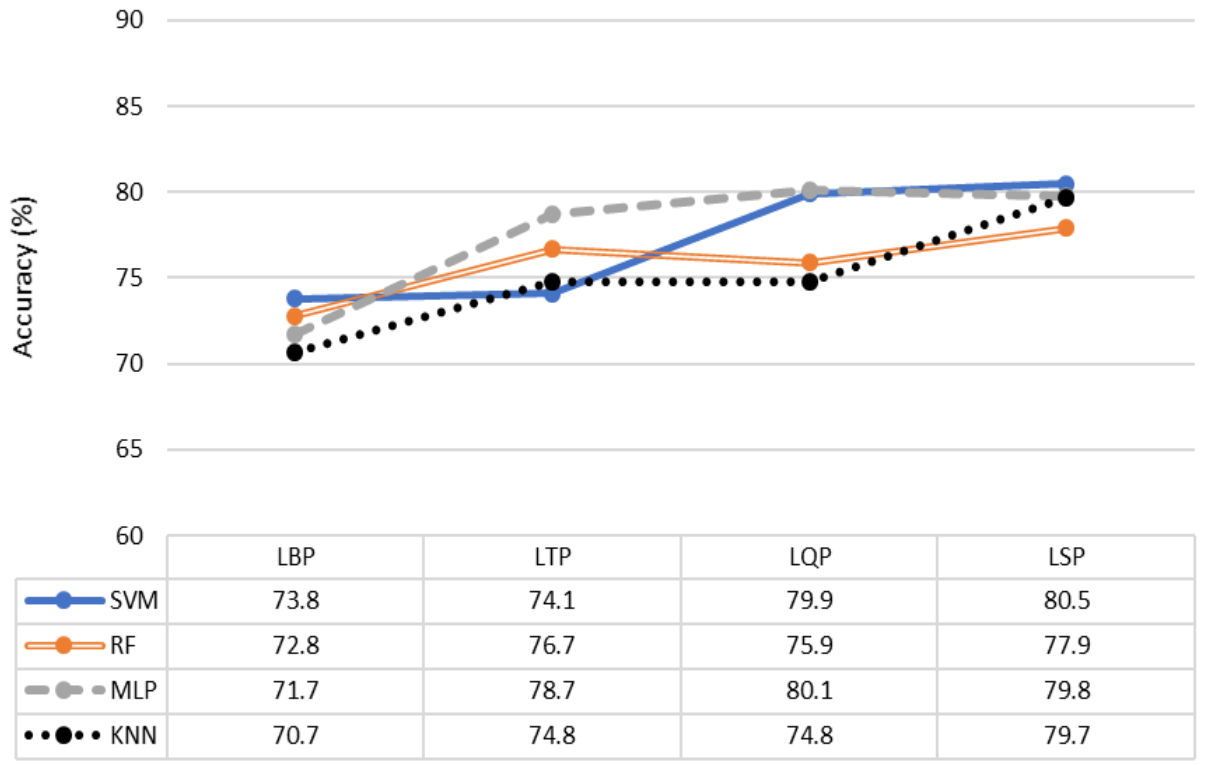

Figure 18: Comparison of maximum accuracies achieved by each operator across different classifiers when evaluated on the InBreast dataset.

\section{Discussion}

In this section we will discuss (i) the effects on accuracy when different numbers of dominant patterns are used in the classification, (ii) the effects of the radius $(R)$ and number of neighbours $(P)$ in the local patterns discriminatory levels; (iii) explanations on why multichannel local patterns produced more discriminant features in comparison to the original LBP operator, in (iv) extending to 9 and 11- encoding systems, (v) discussion of the existing studies in the literature, (vi) statistical analysis and (vii) future work.

\subsection{Effects of Different Numbers of Dominant Patterns (n)}

To investigate the effects of $n$ on the classification accuracy for all operators described in this study we tested 60 different values from 90 to 99.9 (e.g., $n \in\{90,90.1,90.3,90.5, \ldots, 99.9\}$ ). In other words, we investigated the variation of classification accuracy by including local patterns which have a frequency of minimum $0.1 \%$ to $10 \%$ in the histogram feature (e.g. local patterns with a relative frequency of less than $0.1 \%$ to $10 \%$ are removed, resulting to a shorter histogram feature). Figure 20 shows the effects of $n$ 


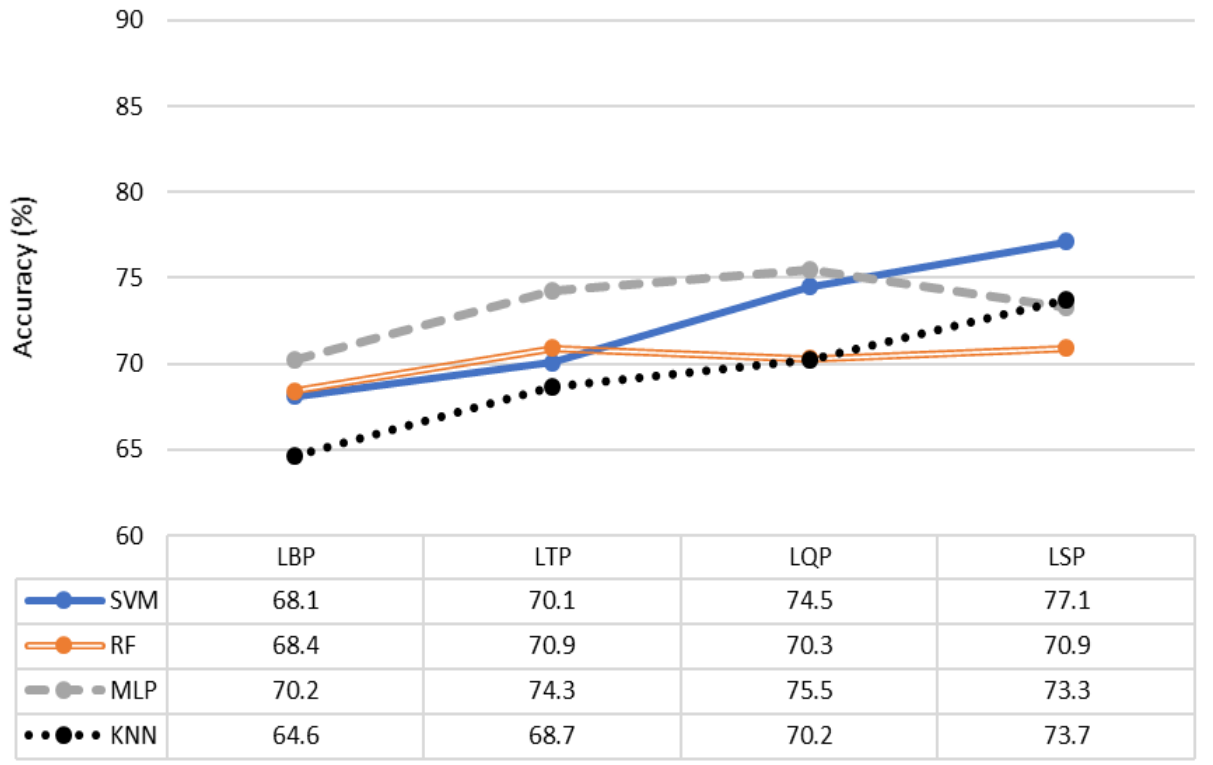

Figure 19: Comparison of average accuracies across different numbers of dominant patterns achieved by each operator across different classifiers when evaluated on the InBreast dataset.

on classification accuracy for LBP, LTP, LQP and LSP operators. In this experiment, we employed the SVM classifier as it produced better performance compared to the MLP, RF and $k$-NN classifiers. It can be observed that $n$ plays an important role in getting the best classification accuracy. For the LBP operator it can be observed that a large variation of classification accuracy between $63 \%$ to $74 \%$ occurs, with a $9.71 \%$ standard deviation. The LQP operator produced a standard deviation of $9.25 \%$ which is higher than the LTP operator of $9.04 \%$. The LSP operator produced a smaller standard deviation value of $9.04 \%$ (the same as LTP) which indicates that our proposed method does not only outperform the other operators on both datasets but also produced more consistent results.

\subsection{Effects of Radius (R) and Number of Neighbours (P)}

In this section we are interested to know to what extent $R$ and $P$ affect the overall classification accuracy. For this purpose, we investigated seven different combination of $R$ and $P$ and tested each of them with all operators. Figures 21 and 22 show average maximum accuracies for both datasets using 


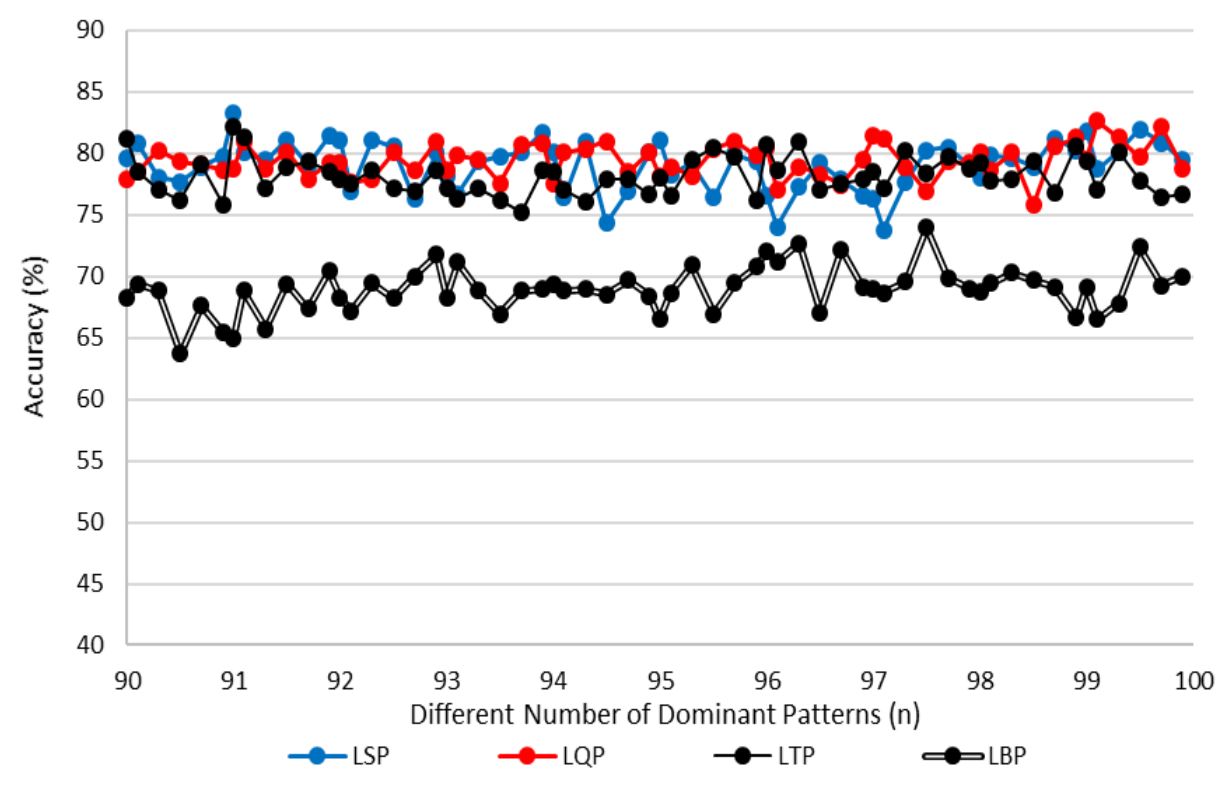

Figure 20: Effects of different numbers of dominant patterns $(n)$ across different operators $((\mathrm{P}, \mathrm{R})=(18,9))$ using SVM classifier on MIAS dataset.

the SVM classifier. For the LBP operator, local patterns extracted using smaller radii and less number of neighbours (e.g., $(5,10)$ and $(5,12))$ tend to produce better results whereas the LTP operator produced its highest average maximum accuracy at $(7,14)(A c c>78 \%)$. Similar results of $77.5 \%$ are obtained when the following parameters are employed: $(7,16),(9,18)$ and $(9,20)$. However, when evaluated on the InBreast dataset, the highest average maximum accuracy $(76 \%)$ is achieved at $(9,18)$ followed by parameters $(7,14)$ and $(7,16)$. For the LQP operator, the highest average maximum accuracy $(A c c>78 \%)$ is achieved at parameters $(7,14)$ followed by $(9,18)$ with only $0.5 \%$ difference. In the InBreast dataset, the LQP operator produced highest average maximum accuracy of $77.5 \%$ with parameters $(9,18)$. The LSP operator produced consistent average maximum accuracy when parameters $(7,14),(7,16),(9,18),(9,20)$ and $(11,22)$ are used which is around $77 \%$. Nevertheless, it produces at least $2.5 \%$ higher average maximum accuracy at $(9,20)$ compared to $(7,14),(7,16),(9,18)$ and $(11,22)$. These results indicate that when choosing the values for $P$ and $R$, the following values $(7,16)$, $(9,18),(9,20)$ are a good starting point.The LSP operator produced its best 
results at $(9,20)$ using the SVM classification approach.

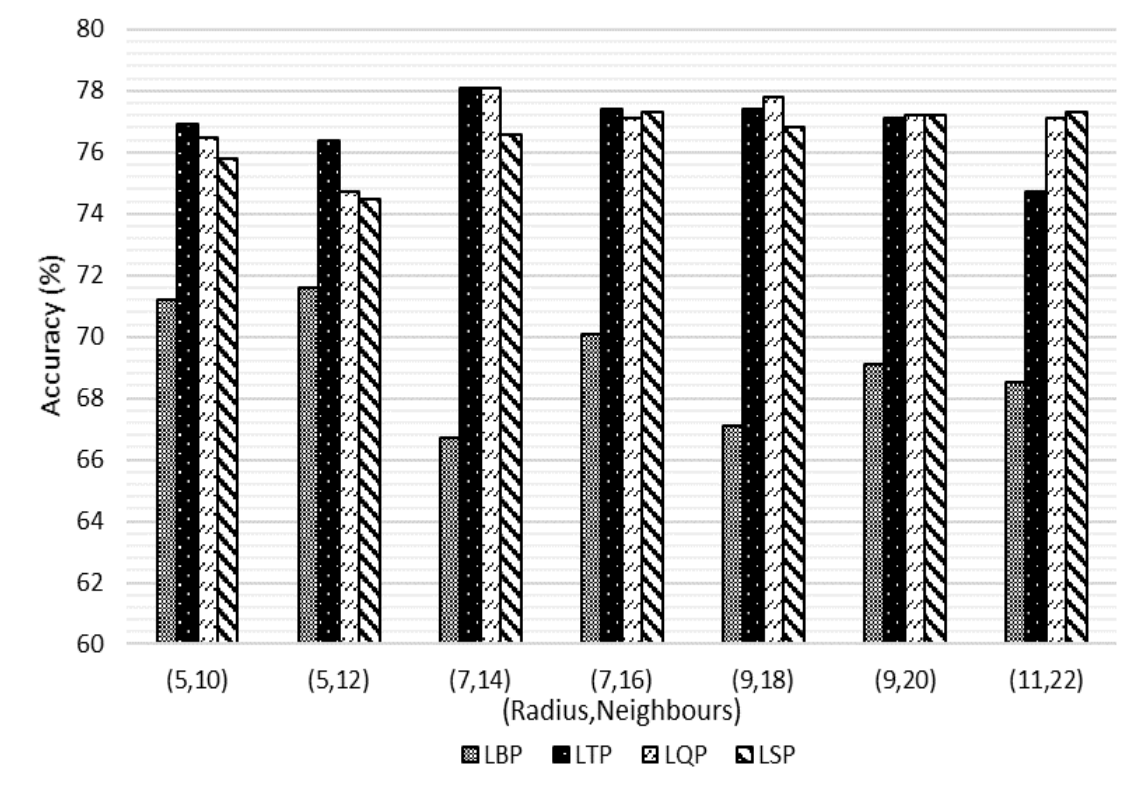

Figure 21: Average maximum accuracy (MIAS dataset) across different classifiers based on different operators and their associated parameters.

\subsection{Why do multichannel local patterns work?}

To answer this question we conducted several experiments by performing channel based classifications on the MIAS dataset. In the case of the LTP operator (three channels with two binary patterns), firstly we perform individual classification based on features extracted from the first binary patterns and secondly we concatenate features extracted from two or more channels. Table 3 shows our experimental results for the LTP, LQP and LSP operators. Note that the following parameters are used based on their best performance using the SVM classifier (see Figures 12, 13 and 14): $\operatorname{LTP}(7,16)$ with $n=93.5, \operatorname{LBP}(9,18)$ with $n=99.9$ and $\operatorname{LSP}(9,18)$ with $n=99.1$.

It can be observed that classification based on features extracted from a single channel alone is insufficient. For the LTP operator, each channel produced just over $73 \%$ but concatenating local patterns from all channels produced $81 \%$, yielding over $7 \%$ improvement. A similar case occurred for the LQP operator where binary patterns from a single channel produced $A c c<70 \%$. However, combining binary patterns from the first three 


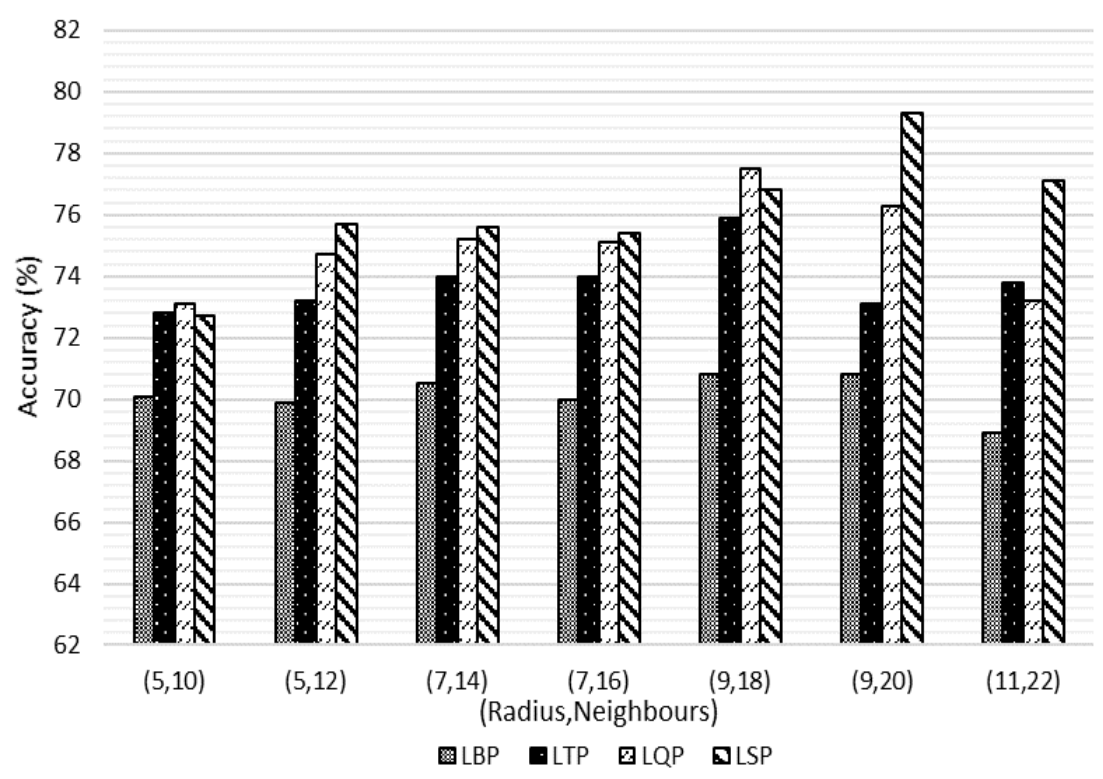

Figure 22: Average maximum accuracy (InBreast dataset) across different classifiers based on different operators and their associated parameters.

channels $(C h 1 \oplus C h 2 \oplus C h 3)$ yields a significant improvement $76.4 \%$ and concatenating all local patterns from all channels produced a maximum accuracy of $82.1 \%$. For our proposed operator, $C h 2$ and $C h 5$ produced classification accuracy under $60 \%$ and $C h 1, C h 3$ and $C h 4$ produced accuracy close to $70 \%$. Concatenating all features from all channels resulted in $A c c=83.3 \%$ however removing a less informative channel (e.g., $C h 2$ ) by considering $C h 1 \oplus C h 3 \oplus C h 4 \oplus C h 5 \oplus C h 6$, the classification accuracy improved to $83.8 \%$.

Based on these experiments we found that encoding an image by dividing it into several channels captures more textural details which is difficult to capture with the original LBP operator. This can be clearly seen in Figures 8 and 9 where most accuracies achieved by the LBP operator are under $70 \%$ whereas the other operators consistently achieved above $75 \%$ accuracy. This also indicates that the LQP and LTP operators capture more details of the Corpus Mammae region in comparison to the LBP operator. Similarly, the LSP operator captures even more details compared to LQP and LTP operators. However, a higher order encoding system such as dividing into nine or eleven channels does not necessarily improve the classification accuracy 


\begin{tabular}{|c|c|c|}
\hline Operators & Channel $(C h)$ & Channels Concatenation $(\oplus)$ \\
\hline LTP & $C h 1=73.3 \pm 8.6$ & $C h 1 \oplus C h 2=81.95$ \\
& $C h 2=74.1 \pm 9.1$ & $C h 1 \oplus C h 2=69.3 \pm 8.3$ \\
\hline \multirow{4}{*}{ LQP } & $C h 1=59.8 \pm 10.6$ & $C h 1 \oplus C h 2 \oplus C h 3=76.4 \pm 8.1$ \\
& $C h 2=65.9 \pm 9.6$ & $C h 1 \oplus C h 2 \oplus C h 3 \oplus C h 4=82.1 \pm 7.1$ \\
& $C h 3=68.9 \pm 11.5$ & \\
\hline \multirow{5}{*}{ LSP } & $C h 4=66.5 \pm 9.9$ & $C h 1 \oplus C h 2=74.3 \pm 7.9$ \\
& $C h 2=52.6 \pm 11.6$ & $C h 1 \oplus C h 2 \oplus C h 3 \oplus C h 4=70.1 \pm 10.1$ \\
& $C h 3=70.1 \pm 8.8$ & $C h 1 \oplus C h 2 \oplus C h 3 \oplus C h 4 \oplus C h 5=77.7 \pm 9.1$ \\
& $C h 5=59.3 \pm 11.3$ & $C h 1 \oplus C h 3 \oplus C h 4 \oplus C h 6=80.7 \pm 10.1$ \\
& $C h 4=65.7 \pm 12.8$ & $C h 1 \oplus C h 3 \oplus C h 4 \oplus C h 5 \oplus C h 6=83.8 \pm 9.8$ \\
& & $C h 1 \oplus C h 2 \oplus C h 3 \oplus C h 4 \oplus C h 5 \oplus C h 6=83.3 \pm 8.8$ \\
\hline
\end{tabular}

Table 3: Classification results (\%) based on binary patterns extracted from individual channel (second column) and concatenated binary patterns from two or more channels

because it increases the number of features yielding more complex decision boundaries. An early indication can be observed based on our experiment results in Table 3 where best accuracy can be achieved by the LSP operator when excluding local patterns from $C h 2$. This reduces the number of features yielding a less complex decision boundaries in a feature space, hence boosting the overall performance of the method.

A channel encoding system also extracts a set of 'weak' features which produce satisfactory results on its own, but when combined they provide good results (similar to ensemble classifiers such as the Random Forests). For example, in Table 3 combining two or more local patterns from different channels always produce better results. This is due to each channel containing unique information which is not available in the other channels and they complement each other when combined. For example, individual classification in LQP produce under $70 \%$ accuracy but improve to over $76 \%$ and $82 \%$ when three and four channels are combined, respectively. Simi- 
larly, all channels from the LSP operator are unable to provide sufficient information of the Corpus Mammae region individually but when combining $C h 1 \oplus C h 3 \oplus C h 4 \oplus C h 5 \oplus C h 6$ we achieved $83.8 \%$ accuracy.

Moreover, the threshold values which are determined automatically enable the operator to capture robust texture information as pixel information is encoded according to the local threshold values rather than a global threshold value as in the LTP and LQP operators. Local threshold values enable the LSP operator to capture textural information based on the topology and intensity distribution of the image and hence are more discriminant and tolerant to noise. In contrast, a global threshold value does not consider intensity distribution of a region. As a result, it may affect the discriminatory level of the local patterns.

\subsection{Extending to 9-encoding and 11-encoding systems}

To further evaluate the performance of different encoding systems, we conducted several experiments by extending the LSP to 9- and 11-encoding systems both on the MIAS and InBreast datasets. Figures 23, 24, 25 and 26 show that the performance is decreasing as we use 9- and 11-encoding systems.

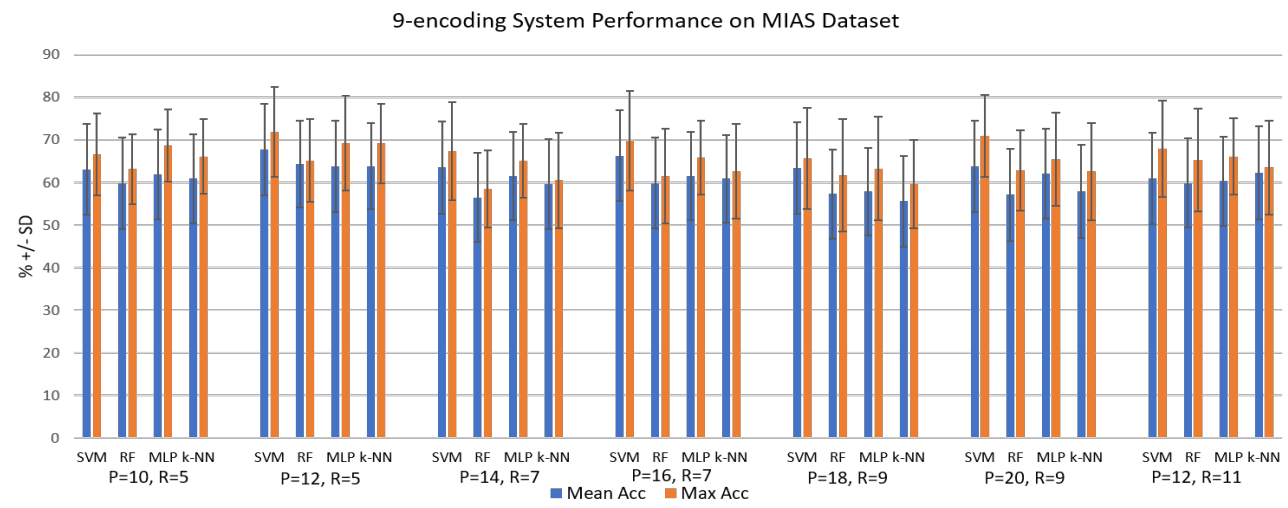

Figure 23: 9-encoding system performance on MIAS dataset

Experimental results suggest that the 7-encoding system the LSP achieved the best performance and eventually decreased to between $55 \%$ to $63 \%$. From our own observation, the main reason for this is due to higher encoding systems (e.g. 9-encoding) producing very sparse features (containing many 
zeros). This is similar when performing the 11-encoding system (in fact features are even sparser). The main problem with sparse features is they are most likely being ignored by most classifiers particularly tree-based machine learning algorithms. Secondly, in most cases zeros are less informative making the features less meaningful and hence less discriminant. As a result, performance classification reduced significantly. In Figure 25 and 26 none of the maximum accuracies achieved was above $70 \%$ whereas the LBP operators achieved above this value in some cases.

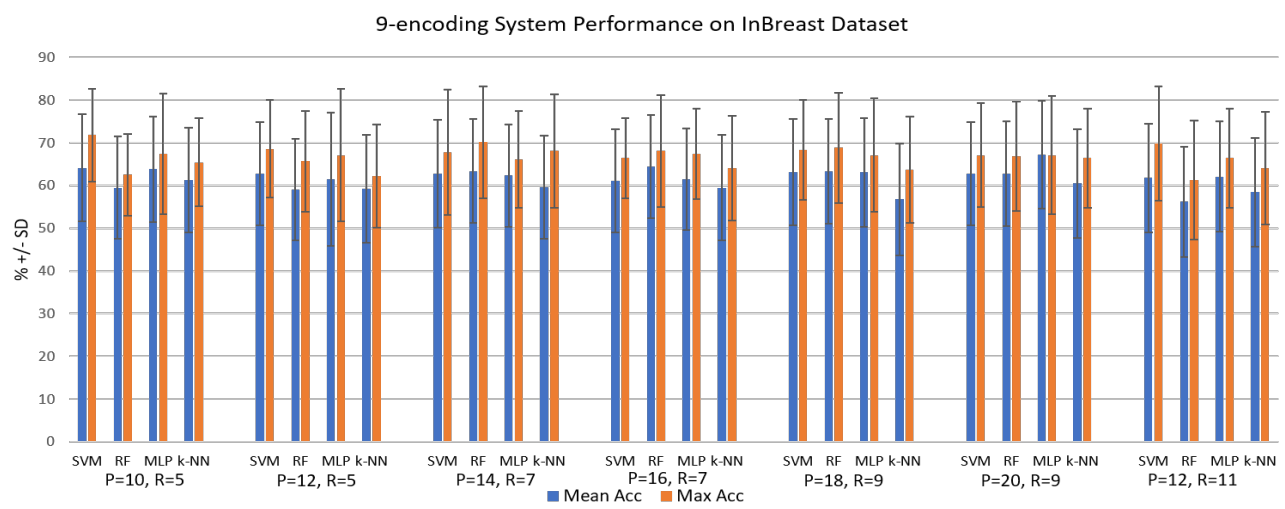

Figure 24: 9-encoding system performance on InBreast dataset

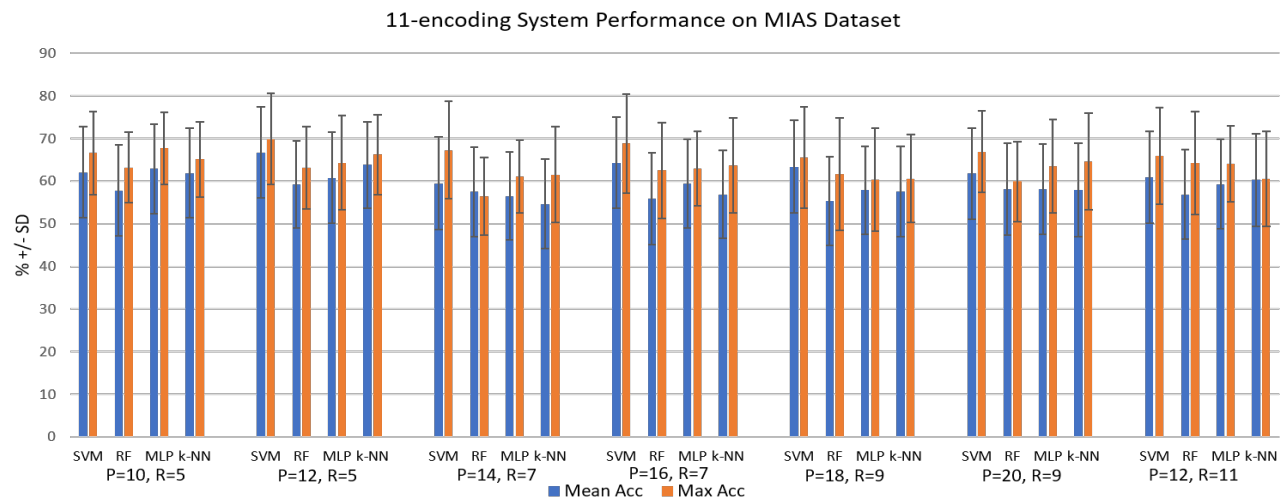

Figure 25: 11-encoding system performance on MIAS dataset 


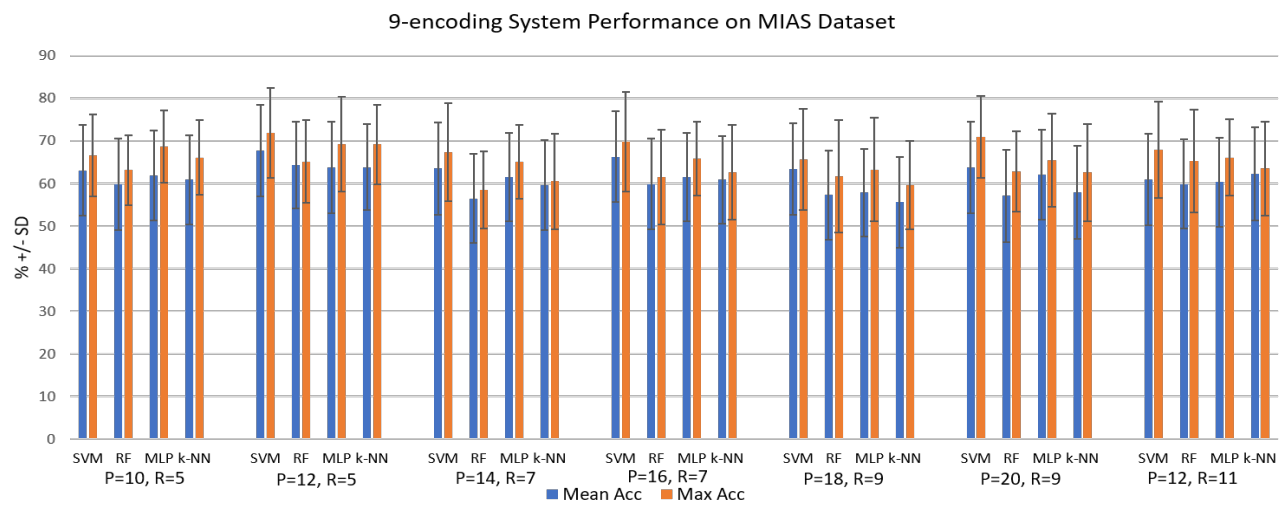

Figure 26: 11-encoding system performance on InBreast dataset

\subsection{Existing Methods in the Literature}

The main goal of our study is to improve the performance of binarybased local pattern feature extraction methods, namely Local Binary Pattern (LBP), Local Ternary Pattern (LTP) and Local Quinary Pattern (LQP). Our study does not intend to quantitatively compare the performance of the LSP operator against other feature extraction methods. There are many feature extraction methods in the literature, therefore quantitative comparison is extremely difficult. It should also be noted that quantitative comparison with the other breast density methods in the literature is because most studies combine features from different feature extraction algorithms such as first-order statistical features, second-order statistical features, morphological features, features of Gabor filters, edge information, etc.

Many breast density classification methods have been developed over the last two decades. The best classification accuracy (based on BI-RADS classes (fourth edition)) reported in the literature is over $90 \%$ by the study by [14] followed by the study of [11] with $86 \%$ classification accuracy. However, these methods are computationally expensive due to different numbers of feature extraction techniques employed. Several techniques used to extract different types of features such as the first order statistical techniques to compute the local pixel intensity, the co-occurrence matrix to extract grey level distribution, an adaptive thresholding technique to extract the region's morphological information, Gabor filter to obtain edges information, etc. Also, their methods employed the Fuzzy C-Means clustering technique as a separate process to classify the breast tissue into different pixel intensities. Once again this 
process is time consuming especially when dealing with large images such as mammograms. In contrast, the LSP operator (with accuracy close to $84 \%$ ) extracts and processes all this information at once, hence reducing the computational complexity of the system.

Previously, [20] used the LTP operator to extract local information and achieved a promising result of $82.33 \%$ accuracy. However, this method suffers from having to deal with a large number of features (over one thousand) due to the multi-orientation approach (e.g., ten histograms from ten orientations concatenated). Our recent study, [21] reported the best accuracy of over $86 \%$ using multi-orientation LQP operator and combining other local patterns extracted using on ellipse neighbourhood. Nevertheless, this method contains several separate stages (e.g. extraction using different neighbourhood topologies (circle and ellipse), different orientations of resolutions which can be tedious whereas the LSP operator uses only one neighbourhood topology (e.g. a circle) with a single resolution. Other studies in the literature such as $[11,12,13,14,15,18,19,22,23]$ reported accuracies under $80 \%$. However, note that the purpose of our study is not to develop a breast density classification method that can necessarily outperform the other methods in the literature but to study the effects of various channel encoding techniques in the original LBP operator which have lead us to a more robust technique called the LSP operator, improving the performance of the LBP, LTP and LQP operators.

\subsection{Statistical Analysis}

We performed statistical analysis to investigate whether there is a significant difference/improvement at $p \leq 0.05$ between the best results achieved by the LSP operator and the other operators discussed in this study on both datasets. For this purpose, we compared the best maximum (Max) and average (Avg) accuracies produced by the LSP with the best results of LBP, LTP and LQP operators. The $p$-value was computed using a $t$-test comparing each result of each operator with the best result achieved by the LSP operator according to their respective metric (e.g., Max Acc or Avg Acc). The size of population for each dataset are 103 (206 images) and 161 (322 images) for the InBreast and MIAS dataset, respectively.

Table 4 shows the $p$-values between the best accuracies achieved by the LSP operator and the other operators for both datasets. In terms of maximum accuracy, there is a significant improvement at $p \leq 0.05$ when using the LSP features in classifying breast density on the MIAS $(p=0.0015)$ and 


\begin{tabular}{|l|l|l|l|l|l|}
\hline Dataset & Results & LBP & LTP & LQP & LSP \\
\hline \multirow{5}{*}{ MIAS } & Max Acc $(\%)$ & $73.8 \pm 10.6$ & $81 \pm 9.5$ & $82.1 \pm 7.1$ & $83.3 \pm 8.8$ \\
& $(p$ value $)$ & $(0.0015)$ & $(0.2260)$ & $(0.4019)$ & - \\
\cline { 2 - 6 } & Avg $A c c(\%)$ & $69.7 \pm 9.7$ & $77.5 \pm 9.2$ & $78.6 \pm 8.3$ & $81.6 \pm 9.1$ \\
& $(p$ value $)$ & $(0.00001)$ & $(0.0428)$ & $(0.0433)$ & - \\
\hline \multirow{5}{*}{ InBreast } & Max Acc $(\%)$ & $73.8 \pm 9.9$ & $78.7 \pm 11.2$ & $80.1 \pm 10.5$ & $80.5 \pm 9.2$ \\
& $(p$ value $)$ & $(0.0064)$ & $(0.2646)$ & $(0.4489)$ & - \\
\cline { 2 - 6 } & Avg $A c c(\%)$ & $70.2 \pm 11.7$ & $74.3 \pm 11.4$ & $75.5 \pm 11.3$ & $77.1 \pm 10.9$ \\
& $(p$ value $)$ & $(0.0007)$ & $(0.0137)$ & $(0.2151)$ & - \\
\hline
\end{tabular}

Table 4: Classification results (\%) and $p$-values between the best accuracies achieved by the LSP operator and the other operators discussed in this study.

InBreast $(p=0.0007)$ datasets. Nevertheless, the best maximum results of the LSP operator are not statistically significant at $p \leq 0.05$ in comparison with the best accuracies achieved by the LTP and LQP operators. The $p$-values are 0.2260 and 0.4019 for the LTP and LQP operators, respectively when evaluated on the MIAS dataset which are similar when tested on the InBreast dataset, $p=0.2646$ and $p=0.4489$ for the LTP and LQP operators, respectively. The results are statistically significant at $p \leq 0.05$ on the MIAS dataset when comparing the best average accuracy produced by the LSP features $(A c c=81.6 \%)$ with the other features with $p$-values are 0.00001, 0.0428 and 0.0433 for LBP, LTP and LQP respectively. Similarly, when tested on the InBreast dataset the best average accuracy produced by the LSP operator is significantly better in comparison to the results of LBP $(p=0.0007)$ and LTP $(p=0.0137)$ operators. However, the best average accuracy of LSP is not significant $(p=0.2151)$ compared to the result of the LQP operator.

\subsection{Limitations of our study}

We highlight the following limitations of our study:

1. The sample dataset used to optimise the classifiers' parameters was taken from the MIAS dataset which only 8-bit contrast. This means the optimised parameters and testing results might be different when 
testing with real clinical data (12 to 14 bits contrast). The reasons we chose the MIAS dataset rather than the InBreast dataset when optimising the classifiers' parameters are (a) it has larger number of images/cases hence giving us a larger number of test cases and (b) the number of cases for each class is more representative compared to the number of cases for each class in the InBreast dataset. For example, the number images of BI-RADS IV is 14 which is extremely small. Including cases in our dataset for parameter optimization, will make our testing dataset extremely imbalanced. Although in this study we used images from the MIAS dataset for parameter optimization, in a real clinical environment this procedure can be easily changed/adapted by using a sub-sample from the new dataset.

2. The MIAS dataset is a somewhat old database and hence does not represent the actual contrast representation of the latest mammograms. We included the MIAS dataset in our study due to (a) it is the most used and compared dataset in the literature because of its availability whereas other datasets are not easily accessible, (b) acquiring mammogram datasets is very difficult especially the ones with BI-RADS (version 4) classification and (c) other datasets do not classify each breast image based on BI-RADS density but are classified based on a small square patch based on tissue types such as 'fatty', 'glandular' or 'breast tissue'.

3. The parameters $P, R$ and $n$ were tested/selected empirically. Nevertheless, our testing strategy is based on the previous studies of Ojala et al. [32], Tan and Triggs [33], Nanni et al. [34]. To the best of our knowledge this a common approach used in the literature especially for parameters $P$ and $R$. For parameter $n$, we tested 60 different values from $n=90$ to $n=99.9$ with 0.2 interval (e.g. 90, 90.1, 90.3, 90.5, etc). This should be sufficient to investigate the performance variations across different $n$ values.

4. The number of cases used in the development of parameter optimization is small. However, we prefer to optimise the number of images for testing purposes and therefore, we limit the number of cases for parameter optimisation. It is more important to test the model performance on a larger size of dataset rather than on a smaller dataset.

\subsection{Future Work}

For future work we plan to consider the following: 
1. Since multichannel based operators such as LTP, LQP and LSP require the number of dominant patterns $(n)$ to obtain an optimum classification accuracy, we plan to develop a statistical method that can automatically determine an optimum value of $n$.

2. We also interested to investigate the performance of LBP's two-channel variants such as M-ELBP, ELBP, MRELBP, STLBP and CLBP when multichanel based operators are being applied.

3. As indicated in Table 3, combining all local patterns from all channels does not necessarily produce optimal accuracy. For example, the LSP operator achieved its highest accuracy when excluding $C h 2$. Therefore, we plan to develop a method that can automatically determine the most informative local patterns from each channel and in the classification phase only combine the most discriminant features. This can be done by measuring mutual information among the histogram features and combining the ones with the least overlapping information.

4. We plan to develop a deep learning based network to extract local features of the Corpus Mammae region and combine them with features extracted with the LQP or LSP operators.

\section{Summary and Conclusions}

In this paper, we studied the effects of various channel encoding techniques in local pattern extraction which have led us to a more robust technique called the LSP operator. We investigated the following aspects in our study:

1. We made comparisons between the LBP, LTP, LQP and LSP operators based on MIAS and InBreast datasets. Experimental results suggest that the proposed texture operator outperformed the other operators on both datasets.

2. We investigated whether the choice of a classifier can influence the performance of the system. We employed four different classifiers and found that in many cases the SVM classifier outperforms the other classifiers. Local patterns extracted with the LTP operator tend to produce better results when the MLP or $k$-NN classifier is employed whereas the SVM classifier produced better accuracy using LQP and LSP features. 
3. We conducted experiments to investigate whether the operators discussed in this study have a particular preference regarding $R$ and $P$. We found that in many cases LBP features produced similar results of under $70 \%$ accuracy. However, the LSP features tend to produce better classification results when $\operatorname{LTP}(7,14)$ and $\operatorname{LTP}(9,18)$ are used. On the other hand, both LQP and LSP operators tend to produce consistent results when $R \in\{7,9\}$ and $P \in\{16,18,20\}$ are used.

4. We studied the effects of selecting different numbers of dominant patterns and experimental results show that the value of $n$ plays an important role in obtaining optimal accuracy. The LSP and LTP operators produced variation accuracy of $9.04 \%$ compared to $9.25 \%$ and $9.71 \%$ for LQP and LBP, respectively.

5. We also studied channel based classification and concatenating local patterns from different channels. Experimental results suggest that each channel contains unique features which are not available in the other channels and combining these features yielded to better classification results. We also found that removing the most uninformative channel in LSP features improves the classification accuracy by $0.5 \%$.

6. We developed simple methods to automatically determine the threshold values in LSP by computing the $25^{\text {th }}$ percentile and $75^{\text {th }}$ percentile of the neighbouring pixels.

In conclusion, we have studied various channel encoding techniques in LBP, LTP and LQP operators where we found that multichannel local patterns are more robust in discriminating different classes of breast density. This study has led us to the development of a seven-encoding technique call LSP operator to capture more texture details within the Corpus Mamae region. We also introduced a simple method to automatically determine the threshold values in the LSP operator by computing the first order statistical values of the neighbourhood pixels. Experimental results show that the LSP features outperformed the LBP, LTP and LQP operators on both MIAS and InBreast datasets. The LSP features produced a maximum accuracy of $83.8 \%$ using the SVM classifier when combining local patterns from $C h 1$, $C h 3, C h 4, C h 5$ and $C h 6$ on the MIAS dataset. The LBP, LTP and LQP features achieved maximum accuracies of $73.8 \%, 81 \%$ and $82.1 \%$, respectively. Based on these experimental results, the proposed seven-encoding system approach (LSP) is shown to be a robust and more consistent texture operator. 


\section{Declaration of competing interest}

None Declared.

\section{Acknowledgment}

This research was undertaken as part of the Decision Support and Information Management System for Breast Cancer (DESIREE) project. The project has received funding from the European Union's Horizon 2020 research and innovation programme under grant agreement No 690238.

\section{References}

[1] American Cancer Society, Breast cancer facts and figures 2017/2018, 2018. Available at https://www.cancer.org/content/ dam/cancer-org/research/cancer-facts-and-statistics/ breast-cancer-facts-and-figures/breast-cancer-facts-and-figures-2017-2018. pdf Accessed: 2017-06-23.

[2] C. E. DeSantis, J. Ma, A. G. Sauer, L. A. Newman, A. Jemal, Breast cancer statistics, 2017, racial disparity in mortality by state., CA: A Cancer Journal for Clinicians 67 (2017) 439-448.

[3] Breast Cancer, UK, Breast cancer care. facts and statistics 2018, 2018. Available at https://www.breastcancercare.org.uk/ about-us/media/press-pack-breast-cancer-awareness-month/ facts-statistics Accessed: 2017-05-23.

[4] Breast Cancer, UK, Breast cancer symptoms, 2018. Available at https://www.cancerresearchuk.org/about-cancer/ breast-cancer/symptoms Accessed: 2017-06-26.

[5] Breast Cancer, U.S. breast cancer statistics, 2016. Available at http: //www.breastcancer.org/symptoms/understand_bc/statistics Accessed: 2017-06-28.

[6] Breastcancer.org, Dense breast, 2018. Available at https://www. breastcancer.org/risk/factors/dense_breasts Accessed: 2017-0628. 
[7] G. Litjens, T. Kooi, B. E. Bejnordi, A. A. A. Setio, F. Ciompi, M. Ghafoorian, J. A. van der Laak, B. van Ginneken, C. I. Sánchez, A survey on deep learning in medical image analysis, Med. Image Anal. 42 (2017) 60-80.

[8] A. Hamidinekoo, E. Denton, A. Rampun, K. Honnor, R. Zwiggelaar, Deep learning in mammography and breast histology, an overview and future trends, Med Image Anal 47 (2018) 45-67.

[9] N. F. Boyd, J. W. Byng, R. A. Jong, E. K. Fishell, L. E. Little, A. B. Miller, G. A. Lockwood, D. L. Tritchler, M. J. Yaffe, Quantitative classification of mammographic densities and breast cancer risk: Results from the canadian national breast screening study, J. Nat. Cancer Inst. 87 (1995) 670-675.

[10] J. Suckling et al., The mammographic image analysis society digital mammogram database, in: Proc. Excerpta Med. Int. Congr. Ser., pp. $375-378$.

[11] A. Oliver, J. Freixenet, R. Martí, E. P. J. Pont, E. R. E. Denton, R. Zwiggelaar, A novel breast tissue density classification methodology, IEEE Trans. Inf. Technol. Biomed. 12 (2008) 55-65.

[12] K. Bovis, S. Singh, Classification of mammographic breast density using a combined classifier paradigm, in: Proc. Int. Workshop on Digital Mammography, Lecture Notes in Computer Science, Springer, 2002, pp. $177-180$.

[13] M. Muštra, M. Grgić, K. Delać, Breast density classification using multiple feature selection, Automatika 53 (2012) 362-372.

[14] N. M. Parthaláin, R. Jensen, Q. Shen, R. Zwiggelaar, Fuzzy-rough approaches for mammographic risk analysis, Intell. Data Anal. 14 (2010) $225-244$.

[15] D. Raba, J. Martí, R. Martí, M. Peracaula, Breast mammography asymmetry estimation based on fractal and texture analysis, in: Proc. Computed Aided Radiology and Surgery, Berlin, Germany, volume 1398. 
[16] H. Li, M. L. Giger, O. I. Olopade, A. Margolis, L. Lan, M. R. Chinander, Computerized texture analysis of mammographic parenchymal patterns of digitized mammograms, Acad. Radiol. 12 (2005) 863-73.

[17] A. Bosch, X. Munoz, A. Oliver, J. Martí, Modeling and classifying breast tissue density in mammograms, in: Proc IEEE Comput. Soc. Conf. Comput. Vis. Pattern. Recognit. (CVPR), volume 2, pp. 15521558 .

[18] Z. Chen, E. Denton, R. Zwiggelaar, Local feature based mammographic tissue pattern modelling and breast density classification, in: Proc. 4th International Conference on Biomedical Engineering and Informatics (BMEI), volume 1, pp. 351-355.

[19] S. Petroudi, T. Kadir, M. Brady, Automatic classification of mammographic parenchymal patterns: A statistical approach, in: Proc. IEEE Conf. Eng. Med. Biol. Soc., volume 1, pp. 798-801.

[20] A. Rampun, P. J. Morrow, B. W. Scotney, R. J. Winder, Breast density classification using local ternary patterns in mammograms, in: Proc. Image Analysis and Recognition, Springer International Publishing, Cham, 2017, pp. 463-470.

[21] A. Rampun, B. W. Scotney, P. J. Morrow, H. Wang, J. Winder, Breast density classification using local quinary patterns with various neighbourhood topologies, J. Imaging 4 (2018) 14.

[22] M. George, A. Rampun, E. Denton, R. Zwiggelaar, Mammographic ellipse modelling towards birads density classification, in: Proc. International Workshop on Breast Imaging (IWDM) 2016: Breast Imaging, pp. $423-430$.

[23] M. George, R. Zwiggelaar, Breast tissue classification using local binary pattern variants: A comparative study (in press), in: Proc. International Workshop on Breast Imaging (IWDM) 2016: Breast Imaging.

[24] T. Kooi, G. Litjens, B. van Ginnekena, A. Gubern-Méridaa, C. I. Sánchez, R. Manna, A. den Heeten, N. Karssemeijer, Large scale deep learning for computer aided detection of mammographic lesions, Med. Image Anal. 35 (2017) 303-312. 
[25] A. Rampun, K. López-Linares, P. J. Morrow, B. W. Scotney, H. Wang, I. G. Ocaña, G. Maclair, R. Zwiggelaar, M. A. González Ballester, I. Macía, Breast pectoral muscle segmentation in mammograms using a modified holistically-nested edge detection network, Medical Image Analysis 57 (2019) 1-17.

[26] A. A. Mohamed, W. A. Berg, H. Peng, Y. Luo, R. C. Jankowitz, S. Wu., A deep learning method for classifying mammographic breast density categories, Med. Phys. 45 (2017) 314-321.

[27] S. Li, J. Wei, C. Heang-Ping, M. A. Helvie, M. A. Roubidoux, Y. Lu, C. Zhou, L. M. Hadjiiski, R. K. Samala, Computer-aided assessment of breast density: comparison of supervised deep learning and featurebased statistical learning, Phys. Med. Biol. 63 (2018) 15.

[28] M. Kallenberg, K. Petersen, M. Nielsen, A. Y. Ng, P. Diao, C. Igel, C. M. Vachon, K. Holland, R. R. Winkel, N. Karssemeijer, M. Lillholm, Unsupervised deep learning applied to breast density segmentation and mammographic risk scoring, IEEE Trans. Med. Imaging. 35 (2016) 1322-1331.

[29] J. Lee, R. M. Nishikawa, Automated mammographic breast density estimation using a fully convolutional network, Med. Phys. 45 (2018) $1178-1190$.

[30] I. C. Moreira, I. Amaral, I. Domingues, A. Cardoso, M. J. Cardoso, J. S. Cardoso, Inbreast: toward a full-field digital mammographic database, Acad. Radiol. 19 (2011) 236-428.

[31] A. Rampun, P. J. Morrow, B. W. Scotney, R. J. Winder, Fully automated breast boundary and pectoral muscle segmentation in mammograms, Artif. Intell. Med. 79 (2017) 28-41.

[32] T. Ojala, M. Pietikainen, T. Maenpaar, Multiresolution gray-scale and rotation invariant texture classification with local binary patterns, IEEE Trans. Pattern Anal. Mach. Intell. 24 (2002) 971-987.

[33] X. Tan, B. Triggs, Enhanced local texture feature sets for face recognition under difficult lighting conditions, IEEE Trans. Image Process. 19 (2010) 1635-1650. 
[34] L. Nanni, A. Luminia, S. Brahnam, Local binary patterns variants as texture descriptors for medical image analysis, Artif. Intell. Med. 49 (2010) 117-125.

[35] L. Liu, L. Zhao, Y. Long, G. Kuang, P. Fieguth, Extended local binary patterns for texture classification, Image Vision Comput. 30 (2012) 86 -99 .

[36] S. Liao, A. C. S. Chung, Face recognition by using enlongated local binary patterns with average maximum distance gradient magnitude, in: Proc. Computer Vision-ACCV 2007, volume 4844, Lecture Notes in Computer Science, 2007, pp. 672-679.

[37] L. Liu, S. Lao, P. W. Fieguth, Y. Guo, X. Wang, M. Pietikäinen, Median robust extended local binary pattern for texture classification, IEEE Trans. Image Process. 25 (2016) 1368-1381.

[38] J. Yang, S. Wang, Z. Lei, Y. Zhao, S. Z. Li, Spatio-temporal lbp based moving object segmentation in compressed domain, in: Proc. IEEE Ninth International Conference on Advanced Video and Signal-Based Surveillance, pp. 252-257.

[39] Z. Guo, L. Zhang, D. Zhang, A completed modeling of local binary pattern operator for texture classification, IEEE Trans. Image Process. 19 (2010) 1657-1663.

[40] Y. Gio, G. Zhao, M. Pietikäinen, Discriminative features for feature description, Pattern Recognit. 45 (2012) 3834-3843.

[41] M. Hall, E. Frank, G. Holmes, B. Pfahringer, P. Reutemann, I. H. Witten, The weka data mining software: an update, ACM SIGKDD explorations newsletter 11 (2009) 10-18. 


\title{
Breast Density Classification in Mammograms: An Investigation of Encoding Techniques in Binary-based Local Patterns
}

\author{
Andrik Rampun ${ }^{\mathrm{a}, \mathrm{b}, *}$, Philip J. Morrow ${ }^{\mathrm{b}}$, Bryan W. Scotney ${ }^{\mathrm{b}}$, Hui Wang ${ }^{\mathrm{c}}$ \\ ${ }^{a}$ Academic Unit of Radiology, Department of Infection, Immunity and cardiovascular \\ Disease, Sheffield University, S10 2RX, UK \\ ${ }^{b}$ School of Computing, Ulster University, Jordanstown, Northern Ireland, BT37 OQB, \\ $U K$ \\ ${ }^{c}$ School of Computing, Ulster University, Jordanstown, Newtownabbey, Northern Ireland, \\ BT37 0QB, UK
}

\begin{abstract}
We investigate various channel encoding techniques when applied to breast density classification in mammograms specifically local binary, ternary and quinary encoding approaches are considered. Subsequently, we propose a new encoding approach based on a seven-encoding technique yielding a new local pattern operator called a local septenary pattern operator. Experimental results suggest that the proposed local pattern operator is robust and outperforms the other encoding techniques when evaluated on the Mammographic Image Analysis Society (MIAS) and InBreast datasets. The local septenary patterns achieved a maximum classification accuracy of $83.3 \%$ and $80.5 \%$ on the MIAS and InBreast datasets, respectively. The closest comparison achieved by the other local pattern operators is the local quinary operator producing $82.1 \%$ (MIAS) and $80.1 \%$ (InBreast) maximum accuracies, respectively.
\end{abstract}

Keywords: Breast mammography, Breast Density, Local Binary Patterns, Local Ternary Patterns, Local Quinary Patterns, Local Septenary Patterns

\footnotetext{
*Corresponding author

Email address: y.rampun@sheffield.ac.uk (Andrik Rampun)
} 


\section{Introduction}

Breast cancer is the leading cause of death in women. Although younger women can also get breast cancer, women over 50 years old have a much higher chance of being affected contributing to over $90 \%$ of the cases [1]. In 2017, according to the recent breast cancer statistics report by [2], more than 250,000 cases of breast cancer were diagnosed in the United States. Breast Cancer UK revealed that over 11,000 women died in the United Kingdom (UK) in 2016 [3]. Although the causes are not fully understood, several risk factors such as age, family history, overweight/obesity and excessive use of alcohol were found to be the risk contributors of the disease. According to the National Health Services (NHS) in the UK, there are several symptoms of breast cancer such as the appearance of lesions, micro-calcification, breast density and change of size and shape of the breast [4]. Nowadays, mammography is the most common imaging technology used for screening breast cancer to find early symptoms of breast cancer. Other alternative technologies are Ultrasound and Magnetic Resonance Imaging (MRI). In current clinical practice, radiologists have to examine each mammogram of a patient to find any signs of abnormality and doctors can tell whether a tumour is benign or malignant through biopsy tests. Although the overall current clinical methods have significantly improved in the last two decades, there are still some deficiencies such as inconsistency among radiologists, and the fact that it is time-consuming and invasive.

Women with a dense breast are six times more likely to develop cancer mainly because a tumour has a similar appearance to dense tissue, making it difficult to detect in mammograms $[5,6]$. Several studies have indicated that there is a significant correlation between breast density and developing breast cancer $[5,6]$. In most cases, dense breasts contain more glandular and fibrous tissue. In mammograms, breast regions with dense tissue tend to have more brighter pixels with fatty tissue usually represented by darker pixels. During the screening procedure, breast density estimation is visually assessed by radiologists and classified into four groups based on the amount of dense tissue. The Breast Imaging-Reporting and Data System (BI-RADS) fourth edition is one of the guidelines used in breast density assessment and contains four main categories:

1. BI-RADS I (0-25\% dense tissues and mostly fatty)

2. BI-RADS II (26-50\% dense tissues with some scattered density) 


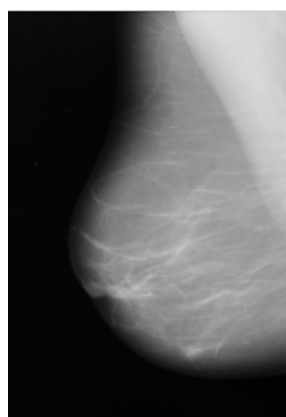

BI-RADS I

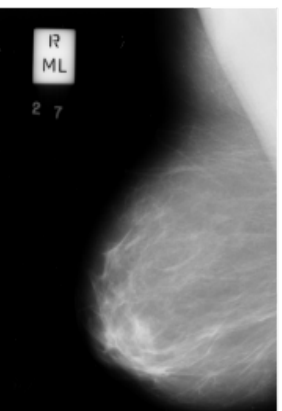

BI-RADS II

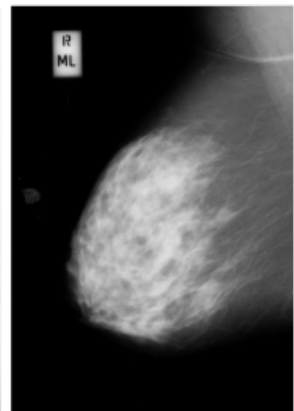

BI-RADS III

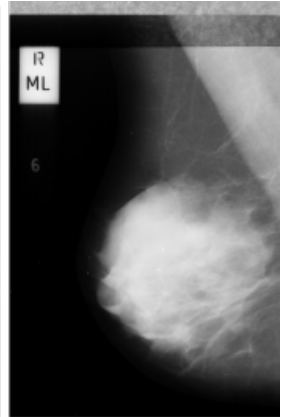

BI-RADS IV

Figure 1: An illustration of breast density according to the BI-RADS guideline fourth edition taken from the the Mammographic Image Analysis Society (MIAS) dataset.

3. BI-RADS III (51-75\% dense tissues and many areas of fibrous and glandular tissue that are evenly distributed)

4. BI-RADS IV (over $75 \%$ dense tissues and breasts have a lot of fibrous and glandular tissue which makes it hard to see a cancer because it can blend in with the normal tissue)

Although manual assessment can be done by radiologists, this task is time-consuming and often suffers from variability between radiologists. For example, radiologists with more experience tend to produce more consistent results compared to less experienced radiologists. Moreover, manual assessment also could lead to false negatives/positives due to fatigue during diagnostic decision making which could influence the final outcome. Figure 1 shows examples of four breasts with their associated BI-RADS classes. In this study we use the fourth edition for simplicity because all datasets are annotated for ground truth based on the BI-RADS fourth edition. However, we are aware that the most recent BI-RADS guidelines is the fifth edition.

\section{Computer-aided diagnosis (CAD) systems}

Computer-aided diagnosis (CAD) systems can assist clinicians regarding efficiency, effectiveness and consistency. CAD systems can assess lesions un-invasively and make predictions as to whether a lesion is benign or malignant. Furthermore, CAD can be used as a 'second reader' to assist clinicians in diagnostic decision making particularly in cases where doctors are not certain about their decision. However, developing a CAD system that can 
replicate radiologists' knowledge requires a significant amount of time and effort. Machine learning is a sub-component of Artificial Intelligence which enables machines to learn and predict a future occurrence of an event. With the use of machine learning and image processing techniques, it is possible to 'teach' computers to learn the appearance/characteristics of breast regions during the training phase and subsequently make a prediction based on the information/knowledge learned.

In breast CAD systems, one of the ways to characterise a breast region in a mammogram is by studying its texture appearance in order to extract meaningful information. In the last decades, many CAD systems for breast imaging have been developed and the majority of them used texture information to investigate the appearance of abnormality such as lesions, dense tissue and micro-calcification clusters. Texture information can be extracted using different mathematical operations such as filter-based, histograms, grey-level distributions, or statistical and probability techniques. Since 2012, the success of deep learning in image classification and segmentation has been overwhelming computer scientists. As a result, hundreds of papers about deep learning for medical image analysis have been published according to the studies by [7] and [8]. Nevertheless, the primary deficiency of deep learning based approaches is the need for a large number of datasets with breast density annotation which can be very challenging in the medical domain. To the best of our knowledge, none of the studies in the literature has attempted using deep learning for four-class breast density classification. The majority of them performed either two-class (low risk (BI-RADS I and II) versus high risk (BI-RADS III and IV)) or three-class (fatty, glandular and dense) classification. On the other hand, many studies have attempted fourclass classification using conventional machine learning in conjunction with texture information.

In this study, we are interested in investigating the effects of various encoding techniques in local pattern extraction by dividing the binary patterns into two (local binary patterns (LBP)), three (local ternary patterns (LTP)) and five (local quinary patterns (LQP)) channels. Subsequently, we propose a local septenary patterns (LSP) operator which divides binary patterns into seven channels. Furthermore, we also study the discriminatory level of local patterns extracted from each channel which can help us to determine more informative texture descriptors. The paper has the following contributions:

1. To the best of our knowledge this is the first study attempting to inves- 
tigate the effects of different encoding techniques covering LBP, LTP and LQP applied to breast density classification.

2. We introduce a variant of LBP (two-encoding technique), LTP (threeencoding technique) and LQP (five-encoding technique) called local septenary patterns (LSP).

3. We introduce several ways of improving local patterns' discriminatory level in the application to breast density classification via a multichannel concatenation approach that enables us to combine local binary patterns from different channels.

4. Whilst threshold values in LTP and LQP need to be set manually by a user, we introduce a simple adaptive way to determine threshold values in LSP based on the intensity distribution of the neighbourhood.

5. Finally we also make a quantitative assessment on each of the channels which can reveal more informative texture descriptors.

Note that our study does not attempt to develop a new CAD system for breast density classification but to investigate the effects on discriminatory levels of local patterns using various encoding techniques.

\section{Breast Anatomy in a Mammogram}

Figure 2 shows breast anatomy in a mediolateral-oblique mammogram (MLO). In many cases, the upper retroglandular region contains fatty tissue and the Corpus Mammae region contains glandular and dense tissue (if the breast is categorised BI-RADS III or IV). Note that usually dense tissue has brighter pixels compared to glandular tissue and these tissues have a similar appearance to the pectoral muscle region. Since many biological activities in the Corpus Mammae region are due to more Lobules and Ducts, dense tissue mostly starts its development in this area.

\section{Literature Review}

Breast density classification in mammograms is one of the most popular topics in breast CAD systems together with mass and micro-calcification clusters classifications. In a majority of the cases, textures have been a popular choice for most authors mainly due to its efficiency and effectiveness. Previously, manual and adaptive thresholding techniques have been used to 


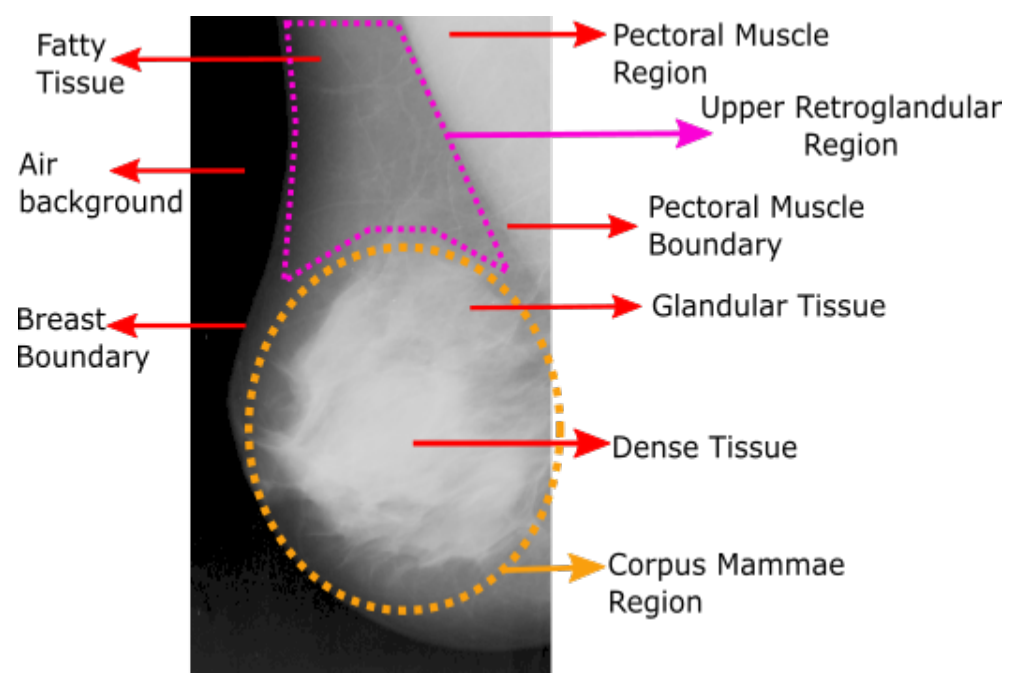

Figure 2: An overview of breast anatomy in a mediolateral-oblique mammogram (MLO). Note that the contrast of the image has been adjusted from the original image for improve visualisation of the breast boundary.

estimate the amount of dense tissue. For example, [9] developed an interactive thresholding software called Cumulus to segment regions with dense tissue by manually tuning a grey-level threshold value. Due to the difficulty in getting annotated mammograms, this topic did not get much attention from computer scientists until the MIAS dataset was made available publicly in 1994 [10].

The first- and second-order (e.g., Grey Level Co-occurrence Matrix (GLCM)) statistical features are among the most popular texture descriptors. For example the studies of [11], [12], [13] and [14] all used these texture descriptors by considering different orientations and resolutions. The methods of [11] and [14] first segment the breast into fatty and non-fatty regions followed by feature extraction on each region and future selection to remove uninformative descriptors. Several machine learning algorithms were employed by [11] and reported an accuracy of $77 \%, 72 \%$ and $86 \%$ for $k$-Nearest Neighbours, C4.5 and Bayesian Network classifiers, respectively. [14] reported an accuracy over $91 \%$ using rough fuzzy approaches with the same feature extraction techniques used by [11]. [13] selected a set of first- and second-order statistical features using a combination of several feature selection techniques and reported a maximum accuracy of $79.2 \%$. [12] extracted features from the 
Spatial Grey Level Dependency matrix (a variant of GLCM) as dense tissue descriptors and employed a combined classifier paradigm based on feedforward Artificial Neural Network (ANN) models to achieve just over $71 \%$ classification accuracy.

Morphology-based descriptors also have been widely investigated to characterise the shape appearance of fatty and non-fatty regions. In many cases, they are used as a set of descriptors with first- and second-order statistical features. For example, [11] and [14] extracted a set of morphology features such as the area, mean, standard deviation and kurtosis using a moment based histogram technique from the segmented fatty and non-fatty regions. The fractal-based feature extraction technique based on a set of threshold values is often used to measure the ratio between the number of low and high grey-levels within a region of interest (e.g., within a $5 \times 5$ neighborhood). For example, [15] divided breast regions by recursively splitting it into quadrants depending on the intensity information that is contained in each region. Subsequently, each subregion is evaluated until the decision function determines that the region does not need to be split, thus obtaining regions with similar properties of tissue. They reported an accuracy of $93 \%$ based on two-class classification (low versus high risk). [16] used fractal analysis to characterise parenchymal patterns in digital mammograms for risk assessment of developing breast cancer, and reported an area under the curve value of 0.86 .

Bag-Of-Words features such as Textons and Scale Invariant Feature Transform (SIFT) have also been studied in the last decade. [17] compared the performance of Textons (extracted from the original images instead of the filtered images) and SIFT features for four-class density classification and used probabilistic Latent Semantic Analysis (pLSA) to obtain a meaningful set of features generating a compact tissue representation of each density class. They reported over $91 \%$ and $88 \%$ accuracies achieved by Textons and SIFT approaches, respectively. In a similar study, [18] compared the performance of various methods for mammographic dense tissue pattern modelling such as the Local Grey-Level appearance (LGA), Textons, LBP and Basic Image Features (BIF). In the Textons approach, [18] used various filter sizes and orientations to extract texture information from filtered images instead of directly from the original images and various parameter values (e.g., window size and the number of neighbours) were tested to extract LGA, LBP, and BIF features. For the four-class BI-RADS classification the authors reported accuracies $75 \%, 72 \%, 59 \%$ and $70 \%$ for Textons, LGA, LBP and BIF, re- 
spectively. [19] implemented a Textons approach based on the Maximum Response 8 (MR8) filter bank. The $\chi^{2}$ distribution was used to compare each of the resulting histograms from the testing set with all the learned histogram models from the training set and reported $75.5 \%$ accuracy.

In recent studies, [20, 21] showed that both LTP (three-encoding approach) and LQP (five-encoding approach) operators can achieve $77 \%$ to $85 \%$ accuracy using a multiscale approach with different neighbourhood topologies and different number of dominant patterns. Instead of extracting texture features from the entire breast region, [20, 21] showed that extracting texture information only from the Corpus Mammea reduces the number of overlapping features and hence produces more distinctive features. The authors showed that this approach was at least $7 \%$ better in terms of classification accuracy compared to classifying breast density by extracting features from the entire breast region. [22] modelled the distribution of breast density based on the multi-scale distribution of dark ellipses (representing fatty tissue) and bright ellipses representing fibroglandular and dense tissues. Subsequently, morphological features such as distances, sizes and diameters were extracted to estimate the amount of dense tissue in the breast. Preliminary results show that their method achieved accuracy between $67 \%$ to $72 \%$ based on various classifiers. Later, [23] compared the performance between Elliptical LBP (ELBP), uniform ELBP and Mean-Elliptical LBP (M-ELBP) for threeclass classification (fatty, glandular and dense) and reported classification accuracies of $75 \%, 74 \%$ and $80 \%$, respectively.

Despite a large number of methods having been developed to address the breast density classification problem in mammograms, only a few studies have achieved accuracies above $80 \%$ and the majority of the methods produced between $70 \%$ to $79 \%$. Moreover, most studies were based on two-class classification (low risk (BI-RADS I and II) versus high risk (BI-RADS III and IV)) or three-class classification (fatty, fatty-glandular and dense-glandular) instead of four-class classification. This might be due to challenging issues such as complex and overlapping tissue appearance as well as ambiguous texture patterns which make it difficult to separate BI-RADS classes based on texture.

In the last a few years, Convolutional Neural Network (CNN) and Deep Residual Learning (ResNet) are among the most popular deep learning techniques which have been used to address classification and segmentation problems in clinical image data $[8,7]$. Many current studies have claimed that deep learning based methods produced superior results achieving accuracy 
similar to human performances $[8,7,24,25]$. In breast density classification, the majority of the studies used deep learning based methods to address either two-class (scattered and heterogeneously density) or three-class classification problems (fatty, glandular and dense tissue). [26] developed a CNN model to distinguish between scattered density and heterogeneously dense tissues using over 22,000 images. [27] used deep learning networks to classify dense and non-dense samples for the purpose of dense tissue segmentation. [28] used unsupervised deep learning to segment dense tissue in mammograms and estimate the risk of developing breast cancer based on the of segmentation result. Recently, [29] reported that their deep learning classification results are correlated well with BI-RADS density assessments by radiologists and comparable with a state of the art algorithm, Laboratory for Individualized Breast Radiodensity Assessment (LIBRA).

Although deep learning based methods have shown promising results, breast density classification based on the BI-RADS four-class assessment guideline (fourth edition) remains a challenging task. This might be due to a lack of annotated data and the complexity of the task itself. According to the recent study of [8], the main challenge of employing a deep learning network is the requirement of a large dataset and annotations from experts. In addition, deep learning suffers from a lack of direct human interpretability because deep learning features rely on filter responses solicited from a large amount of training data whereas hand-crafted features such as those extracted from local patterns provide transparent information, which are more interpretable to clinicians and researchers. Furthermore, all datasets available publicly suffer from an imbalance in the number of images for each class. For example, in the MIAS [10] and InBreast [30] datasets the number of BI-RADS IV cases is less than $10 \%$ of the total number of cases. As a result, deep learning networks may suffer from an imbalanced classification problem.

\section{Methodology}

This section explains the technical details of our study covering steps involved in the pre-processing, feature extraction and the classification phase. Figure 3 shows a general overview of the workflow in our experiments.

Firstly, we performed breast segmentation to separate the breast boundary and pectoral muscle using the method proposed by [31]. This ensures that only local patterns within the breast region will be extracted. Follow- 


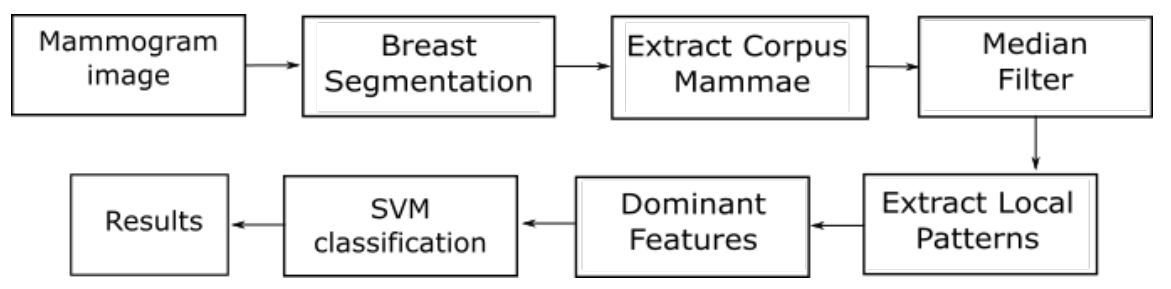

Figure 3: An overview of the work flow involved in our experiments.

ing our previous studies [20,21], we extract the Corpus Mammae region of the breast to reduce the risk of overlapping local patterns across BI-RADS classes. Subsequently, we used a median filter around $3 \times 3$ neighbouring pixels for noise reduction. We extract local patterns at different channels using the LBP, LTP, LQP and LSP operators within the estimated Corpus Mammae region. We select a set of dominant patterns to remove uninformative features and employ the Support Vector Machine (SVM) as a classification approach after a zero-mean normalisation.

\subsection{Pre-processing}

To segment the breast region, we used our previously developed method [31] to estimate the breast and pectoral muscle boundaries. Based on the estimated boundaries we created a breast mask and performed a pixel wise multiplication with the original image resulting in an image with only the breast region. The method [31] employs a region-based Active Contour to estimate the breast boundary and restricted contour growing with edge information for the pectoral muscle boundary estimation. Figure 4 shows an example of an extracted Corpus Mammae region of interest $\left(R O I_{c m}\right)$ and a few examples of estimated breast and pectoral muscle boundaries.

The left-most image in Figure 4 shows the estimated $R O I_{c m}$ area (amber square box). To extract $R O I_{c m}$, we find the height $\left(B_{h}\right)$ and the width of the breast $\left(B_{w}\right) . B_{h}$ is then relocated to the middle of $B_{w}$ to get the intersection point. The width and height of the square area of the $R O I_{c m}$ (amber line Figure 4) can be computed as $B_{w} \times B_{w}$ with the centre located at the intersection point between the $B_{h}$ and $B_{w}$ lines. $B_{h}$ is the height of the breast, which is the longest perpendicular distance between the $x$-axis and the breast boundary. Note that the size of $R O I_{c m}$ varies depending on the width of the breast and local patterns are only extracted within $R O I_{c m}$ instead of from the whole breast region as the majority of the studies in the literature have 


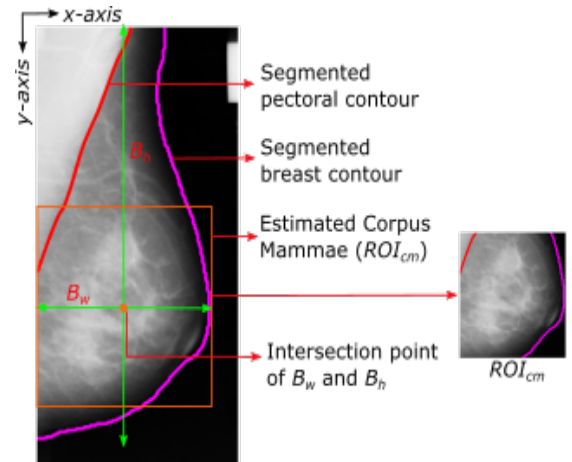

Example of extracted Corpus Mammae

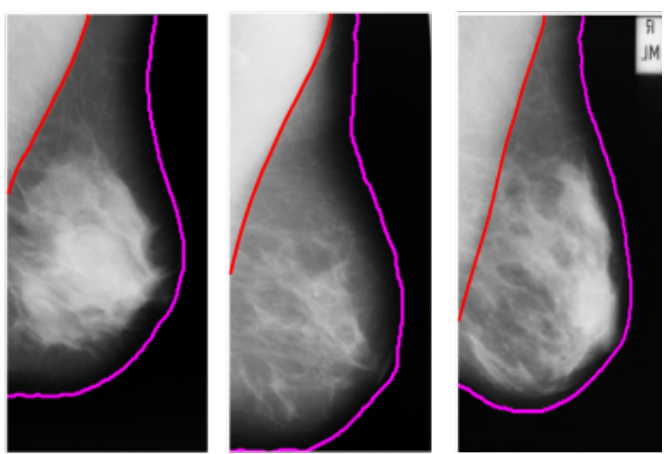

Examples of breast segmentation

Figure 4: Left: an example of extracted Corpus Mammae. Right: examples of breast segmentations.

done.

\subsection{Feature Extraction}

This section explains the original LBP operator [32] and its extensions which are the LTP and LQP operators introduced in [33] and [34], respectively. Subsequently, we propose a LSP operator and explain the different channels in each of the operators. Note that there are several other variants of the LBP operator such as ELBP [35], M-ELBP [36], Median Robust Extended Local Binary Pattern (MRELBP) [37], spatio-temporal LBP (STLBP) [38] and completed LBP (CLBP) [39]. However, they are outside the scope of our study because these operators are based on the LBP's two-channel encoding technique (nevertheless we consider this as part of our future work) whereas the scope of our study is to investigate the effects of local patterns on accuracy when various different different channel encoding techniques are employed.

All these operators use three different mapping tables namely uniform pattern ('u2'), rotation invariant pattern ('ri') and both uniform and rotation invariant patterns ('riu2') to extract uniform, rotation invariant and rotation invariant uniform patterns, respectively. In our study, we investigate the 'riu2' patterns (a combination of ' $\mathrm{u} 2$ ' and 'ri' patterns) as they provide more discriminant features based on our previous studies $[20,21]$ and the study of 
[32]. The value of the LBP code of the pixel $(i, j)$ is given by:

$$
L B P_{(P, R)}(i, j)=\sum_{p=0}^{(P-1)} s\left(g_{p}-g_{c}\right) 2^{p}
$$

where $R$ and $P$ are the radius of the circle that forms the neighbourhood of the operator and the number of pixels in the neighbourhood, respectively. The grey level value of the centre pixel is denoted $g_{c}$, and $g_{p}$ is the grey level value of the $p^{t h}$ neighbour. The LBP operator thresholds the neighbouring pixels using a two-value encoding system as shown in Equation 2.

$$
s(x)= \begin{cases}1, & x \geq 0 \\ 0, & \text { otherwise }\end{cases}
$$

Later, [33] introduced a three-value encoding technique (LTP operator) which thresholds the neighbouring pixels based on a constant threshold set by the user $\left(\tau_{1}\right)$ as shown in Equation 3. Once the LTP code image is generated, it can be separated into two binary patterns from its positive and negative channels. Therefore, it encodes an image into three channels but producing two binary patterns.

$$
s(x)= \begin{cases}1, & x>g_{c}+\tau_{1} \\ 0, & g_{c}-\tau_{1}<x<g_{c}+\tau_{1} \\ -1, & x<g_{c}+\tau_{1}\end{cases}
$$

In [34] the LQP operator was introduced, which thresholds the neighbouring pixels using a five-value encoding technique (see Equation 4) based on two constant thresholds $\tau_{1}$ and $\tau_{2}$. Subsequently, the LQP code image is split into four binary patterns by considering its positives, zero and negative components. Therefore, the LQP operator encodes an image into five channels but results four binary patterns (from two positive channels $(s(1)$ and $s(2))$, zero channel $(s(0))$ and combined negative channels $(s(-1) \cap s(-2)))$.

$$
s(x)= \begin{cases}2, & x \geq g_{c}+\tau_{2} \\ 1, & g_{c}+\tau_{1} \leq x<g_{c}+\tau_{2} \\ 0, & g_{c}-\tau_{1} \leq x<g_{c}+\tau_{1} \\ -1, & g_{c}-\tau_{2} \leq x<g_{c}-\tau_{1} \\ -2, & \text { otherwise }\end{cases}
$$




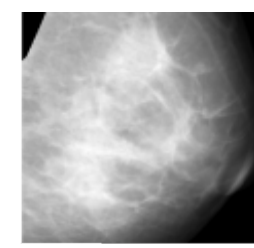

$R O I_{\mathrm{cm}}$

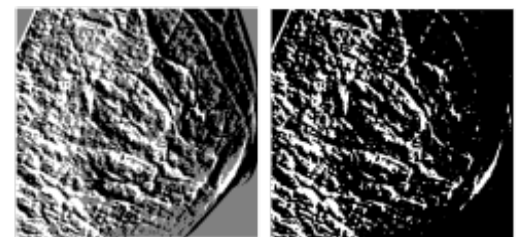

LTP code image LTP Channel 1

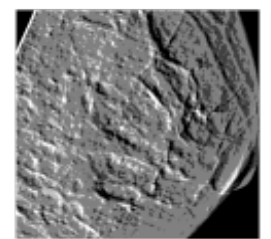

LQP code image

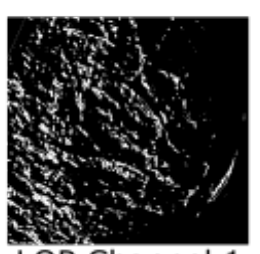

LQP Channel 1
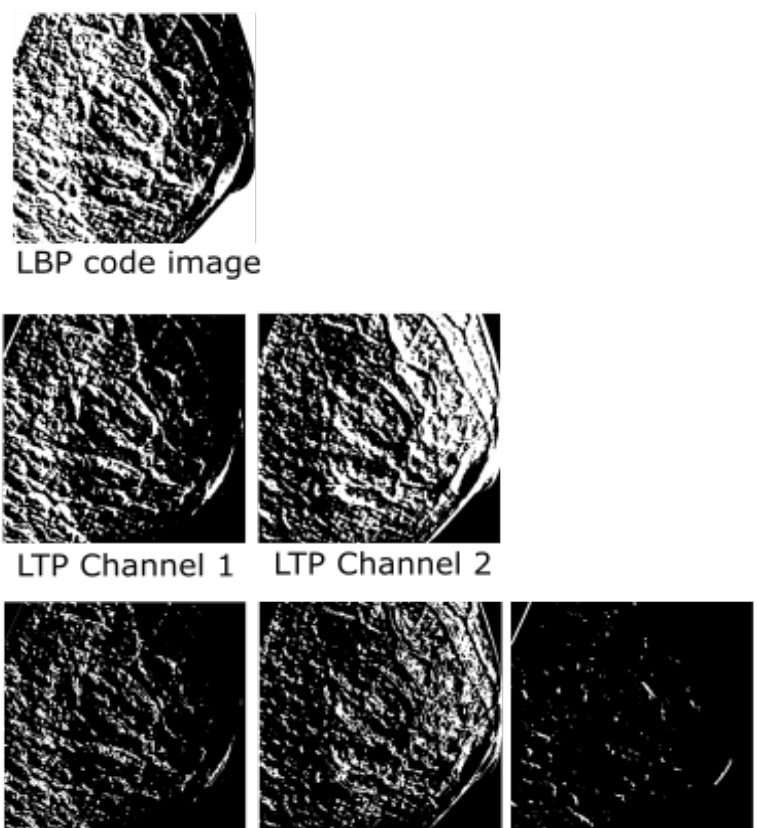

LQP Channel 2

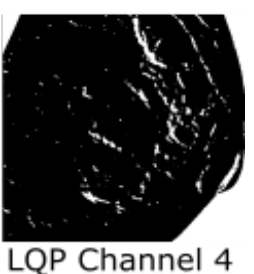

Figure 5: Examples of LBP, LTP and LQP code images and local pattern images from different channels.

We took a similar approach by thresholding the neighbouring pixels using a seven-value encoding technique (hence the name is septenary) based on three threshold values $\left(\tau_{1}, \tau_{2}\right.$ and $\left.\tau_{3}\right)$. The value of the LSP code of the pixel 
$(i, j)$ is given by:

$$
L S P_{(P, R)}^{\text {pattern }}(i, j)=\sum_{p=0}^{(P-1)} s_{\text {pattern }}\left(g_{p}\right) 2^{p}
$$

where pattern $\in\{1,2,3,4,5,6\}$ represents six binary patterns by considering its upper-positive, middle-positive, lower-positive, upper-negative, middlenegative and lower-negative components denoted as $s(3), s(2), s(1), s(-1)$, $s(-2)$ and $s(-3)$, respectively, as shown in Equation 6

$$
s(x)= \begin{cases}3, & x \geq g_{c}+\tau_{3} \\ 2, & g_{c}+\tau_{2} \leq x<g_{c}+\tau_{3} \\ 1, & g_{c}+\tau_{1} \leq x<g_{c}+\tau_{2} \\ 0, & g_{c}-\tau_{1} \leq x<g_{c}+\tau_{1} \\ -1, & g_{c}-\tau_{2} \leq x<g_{c}-\tau_{1} \\ -2, & g_{c}-\tau_{3} \leq x<g_{c}-\tau_{2} \\ -3, & \text { otherwise }\end{cases}
$$

Note that LSP encodes an image into seven channels but results in six binary patterns. The LSP code image is split into six binary patterns using the following conditions

$$
\begin{aligned}
& s_{1}(x)= \begin{cases}1, & \text { if } s(3)=3 \\
0, & \text { otherwise }\end{cases} \\
& s_{2}(x)= \begin{cases}1, & \text { if } s(x)=2 \\
0, & \text { otherwise }\end{cases} \\
& s_{3}(x)= \begin{cases}1, & \text { if } s(x)=1 \\
0, & \text { otherwise }\end{cases} \\
& s_{4}(x)= \begin{cases}1, & \text { if } s(x)=-1 \\
0, & \text { otherwise }\end{cases} \\
& s_{5}(x)= \begin{cases}1, & \text { if } s(x)=-2 \\
0, & \text { otherwise }\end{cases}
\end{aligned}
$$




$$
s_{6}(x)= \begin{cases}1, & \text { if } s(x)=-3 \\ 0, & \text { otherwise }\end{cases}
$$

Figure 6 shows an example of a LSP code image extracted from $R O I_{c m}$ in Figure 5 and its local patterns from different channels. Using the encoding technique in Equation 6, we can generate the LSP code image of $R O I_{\mathrm{cm}}$. The LSP code image is split into six binary images which represent local patterns in six channels. Unlike LTP and LQP where the user has to manually determine threshold values, we introduce an automatic approach by computing the number of neighbours with grey level value $\leq 25^{\text {th }}$ percentile of the entire neighbourhood, number of neighbours with grey level value between $25^{\text {th }}$ and $75^{t h}$ percentile of the entire neighbourhood and number of neighbours with grey level value $\geq 75^{\text {th }}$ percentile. Subsequently, we sort the values in ascending order for $\tau_{1}, \tau_{2}$ and $\tau_{3}$ values.

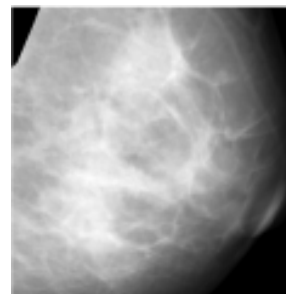

$R O I_{C m}$

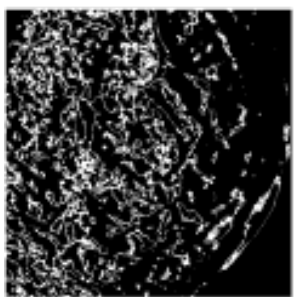

LSP Channel 3

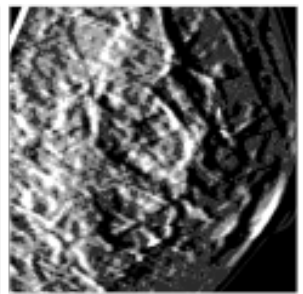

LSP code image

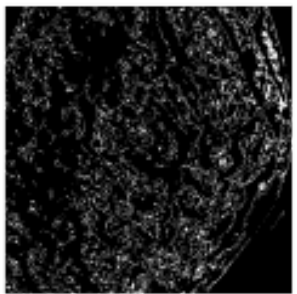

LSP Channel 4

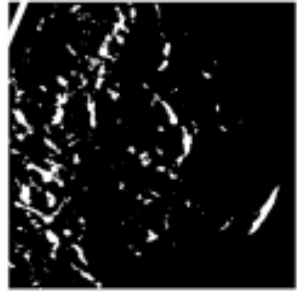

LSP Channel 1

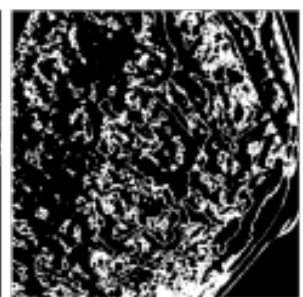

LSP Channel 5

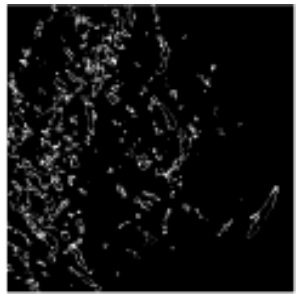

LSP Channel 2

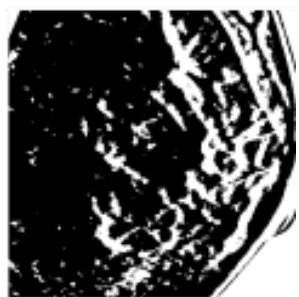

LSP Channel 6

Figure 6: Examples of LSP code image and its local pattern images from different channels.

The LBP, LTP, LQP and LSP are similar in terms of architecture as each is defined using a circle centred on each pixel and the number of neighbours. The main difference is the LSP thresholds the neighbouring pixels into seven $(3,2,1,0,-1,-2$ and -3$)$ values. In contrast, the LBP, LTP and LQP encode an image into two (1 and 0$)$, three (1,0 and -1), and five $(2,1,0,-1$ and -2$)$ values. Figure 7 shows an example of the feature extraction process. The final 
histogram is a concatenation of six histograms computed from each binary pattern (binary image, e.g., LSP code 1) generated based on the conditions in equations (7) to (12). The process of capturing local patterns of $R O I_{c m}$ is also the same for the other operators such as the LBP, LTP and LQP, except less number of channels (resulting in a shorter concatenated histogram).

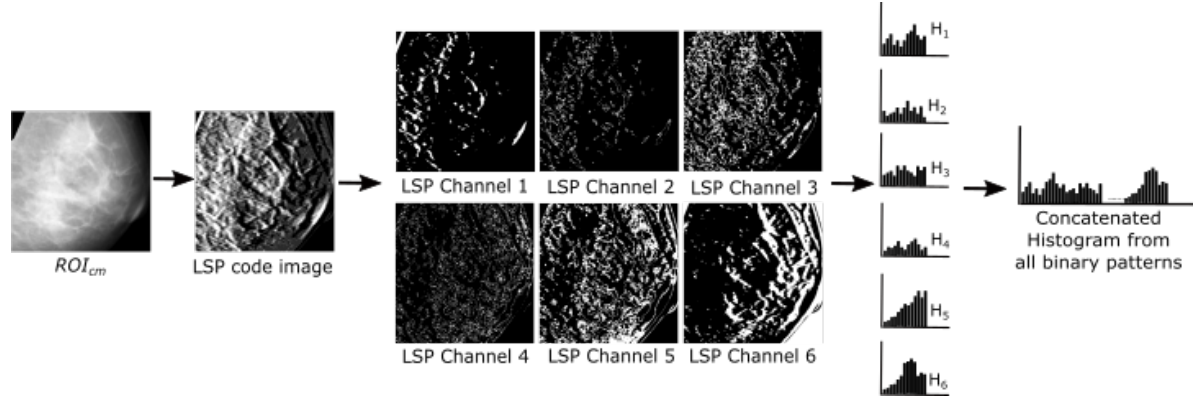

Figure 7: Summary of feature extraction phase using the LSP operator. Note that the binary pattern from each channel is computed from the LSP code image and a histogram image is computed for each channel. Finally, all histograms are concatenated and treated as a feature vector to represent the local pattern of $R O I_{c m}$.

\subsection{Dominant Patterns}

Following our previous studies in $[21,20]$, we selected dominant patterns to reduce feature dimension, hence simplifying the prediction model. Dominant patterns are patterns that occur frequently in the training set [40]. Let $I_{1}, I_{2} \ldots I_{j}$ be images in the training set. We compute the histogram feature $\left(H_{I_{j}}^{L S P}\right)$ for each training image and perform a bin-wise summation for all the histograms to find the pattern's distribution from the training set. Subsequently, the resulting histogram $\left(H^{L S P}\right)$ is sorted in descending order, and the patterns corresponding to the first $D$ bins are selected using the following equation:

$$
D=\arg \min _{N} \frac{\sum_{i=1}^{N-1} H^{L Q P}(i)}{\sum_{i=1}^{2^{P}} H^{L Q P}(i)}>0.01 \times n
$$

where $N$ and $n$ are the total number of patterns and the threshold chosen by the user, respectively. For example choosing $n=98$ means removing patterns that occur with relative frequency of less than $2 \%$ in $H^{L S P}$. The smaller the value of $n$, the smaller the number of patterns selected. 


\subsection{Classification}

Once the feature extraction is completed, we employed several machine learning algorithms in WEKA [41] to build our classification models. For machine learning with only one parameter (e.g., $k$-NN), the CVParameterSelection technique was employed. In contrast, the GridSearch technique was used to explore two parameters for classifiers with two parameters (e.g. RF and SVM). The CVParameterSelection finds the best setting based on options set by the user by optimising the classification accuracy. The GridSearch tests all possible combination of two parameters and selects the best combinations based on the highest accuracy. To find the best parameters for each classifier, 25 patients $(9,7,5,4$ patients for BI-RADS I, II, III and IV, respectively) were selected from the MIAS dataset and 3-fold cross-validation was used to evaluate the performance for each (or pair) of the tested parameter(s) during the optimisation process. The classifier was trained, and in the testing phase, each unseen $R O I_{c m}$ from the testing set is classified as BI-RADS I, II, III or IV. The classifiers used in this study are presented in Table 1.

Table 1: List of classifiers used in this study. The \# sign represents number

\begin{tabular}{ll} 
Classifiers & Default parameters in WEKA \\
\hline Support Vector Machine $(\mathrm{SVM})$ & Complexity $(C)=1.0$, exponent $=1$ \\
Random Forest $(\mathrm{RF})$ & \# of forests $=100$, depth $=0$ (unlimited) \\
Multilayer Perceptron $(\mathrm{MLP})$ & Learning rate $=0.3$, momentum $=0.2$ \\
$k$-Nearest Neighbours $(k-\mathrm{NN})$ & $k=1$, Euclidean distance
\end{tabular}

\section{Experimental Results}

To test the performance of the method we used the following datasets: (a) the Mammographic Image Analysis Society (MIAS) dataset [10] which consists of 322 mediolateral-oblique (MLO) mammograms of 161 women. Films were taken from the UK National Breast Screening Programme and have been digitised to $50 \mu \mathrm{m} \times 50 \mu \mathrm{m}$ and quantised to 8 bits. The distribution for BI-RADS classes is 60 (BI-RADS I), 105 (BI-RADS II), 129 (BI-RADS III) and 31 (BI-RADS IV), and (b) the InBreast dataset [30] which consists of 206 MLO mammograms from 103 patients. Each image is direct digital acquisition on a-Se imaging plates. The pixel size of all images is $70 \mu \mathrm{m} \times$ 
$70 \mu \mathrm{m}$, with 14-bit contrast resolution. The density distribution for the BIRADS classes is as follow: 69 (BI-RADS I), 74 (BI-RADS II), 49 (BI-RADS III), and 14 (BI-RADS IV). Each image contains BI-RADS information (e.g., BI-RADS class I, II, III or IV) provided by an expert radiologist based on the fourth edition of the BI-RADS system. We ran stratified 5-fold cross validation for 10 times.

Accuracy $(A c c)$ is used to measure the performance of the method, which represents the total number of correctly classified images as a proportion of the total number of images.

\subsection{Optimised Parameters}

Table 2 shows a list of parameter values tested and optimised values for the four classifiers employed in this study. Note that, the parameter optimisation was performed based on 25 patients (each BI-RADS class has six or seven patients) taken from the MIAS dataset. Subsequently, we use these parameter values in the testing phase for both MIAS and InBreast datasets. However, for the MIAS dataset we only use 136 patients (272 mammograms) and we excluded 25 patients (50 mammograms) which were used for parameter optimisation. From now on, all parameter settings for the classifiers employed in this study are based on the best parameter values summarised in Table 2.

Table 2: List of parameter options tested in this study.

\begin{tabular}{|c|c|c|}
\hline Classifiers & Parameter tested & Best parameters \\
\hline SVM & $\begin{array}{l}\text { Kernel }=\text { 'Polynomial', } \\
C=1 \text { to } 10 \\
e=1 \text { to } 5\end{array}$ & $C=5, e=1$ \\
\hline $\mathrm{RF}$ & $\begin{array}{l}\text { Number of forests }(r F)=1 \text { to } 165 \\
\text { depth }(D)=0 \text { to } 10\end{array}$ & $r F=70, D=0$ \\
\hline MLP & $\begin{array}{l}\text { Learning rate }(L R)=0.1 \text { to } 2.0(\text { e.g. } 0.1,0.2 \ldots 2.0) \\
\text { momentum }(M)=0.1 \text { to } 2.0(\text { e.g. } 0.1,0.2 \ldots 2.0)\end{array}$ & $L R=0.1, M=0.5$ \\
\hline$k-\mathrm{NN}$ & $\begin{array}{l}k=1-49(\text { e.g. } 1,3,5, \ldots 49) \\
\text { Euclidean distance }\end{array}$ & $k=5$ \\
\hline
\end{tabular}

\subsection{Quantitative Results}

This section presents classification results for LBP, LTP, LQP and LSP operators based on different classifiers. Since the LTP and LQP require 
threshold values from a user, the parameters $\tau_{1}$ and $\tau_{2}$ were selected based on previous studies $[21,20]$. Therefore the $\tau_{1}$ value for the LTP operator is set to 5 and $\tau_{1}$ and $\tau_{2}$ values for LQP are set to 5 and 12, respectively. Note that the average $A c c$ represents the mean accuracy across different numbers of dominant patterns $(n)$ where the maximum Acc is the highest accuracy achieved for $n$ number of dominant patterns. Choosing $n=93$ means removing patterns that occur with a frequency of less than $7 \%$ in the histogram feature. Numerical values represent performance evaluation based on 272 and 206 images for the MIAS and InBreast datasets, respectively. Note that we have used 50 images (of the original 322 images) from the MIAS dataset for parameter optimisation.

Figure 8 and 9 shows classification results using LBP operator for the MIAS and InBreast dataset, respectively. It can be observed that for the MIAS dataset a maximum accuracy of $73.8 \%$ is achieved by $\operatorname{LBP}(7,16)$ (where $R=7, P=16$, e.g. $\operatorname{LBP}(R, P)$ ) using the SVM classifier at $n=91.5$ (removing local patterns with frequency of less than $8.5 \%$ in the histogram features). The best average accuracy of $69.7 \%$ is achieved when employing $\operatorname{LBP}(7,16)$ with $n=92.1$. For the InBreast dataset a maximum accuracy of $73.8 \%$ is achieved by $\operatorname{LBP}(5,10)$ at $n=96.5$ whereas best average accuracy $(70.2 \%)$ is achieved by $\operatorname{LBP}(9,20)$ using the MLP classifier. Overall, the majority of the classification accuracies fall in a range between $65 \%$ to $70 \%$.

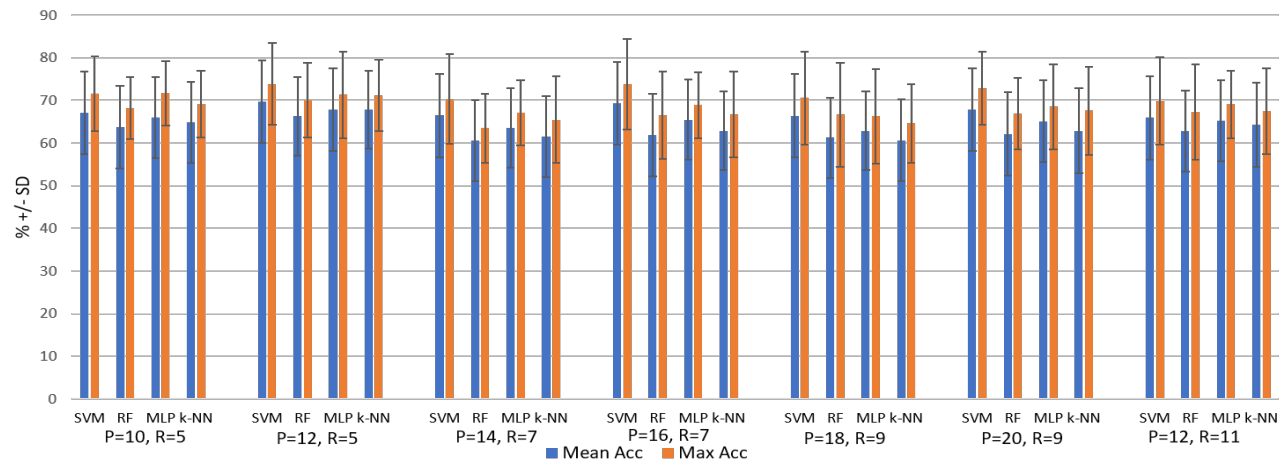

Figure 8: LBP performance on MIAS dataset

Figure 10 and 11 shows quantitative results using the LTP operator for the MIAS and InBreast dataset, respectively. The majority of the average accuracies are in a range between $70 \%$ to $75 \%$ which are higher than the 


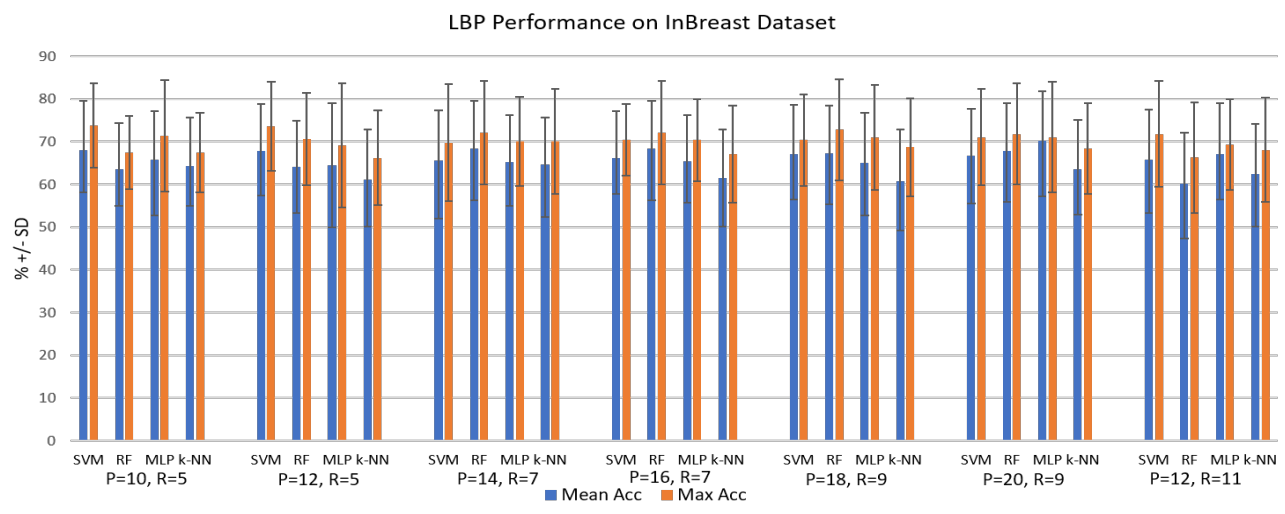

Figure 9: LBP performance on InBreast dataset

ones produced by the LBP operator; for maximum accuracy, many cases are over $75 \%$. Furthermore, it can be observed that the LTP operator outperformed the LBP operator regardless of the $R$ and $P$ values. The highest accuracies are $81 \%$ (average $77.5 \%$ ) and $78.7 \%$ (average $74.3 \%$ ) on the MIAS and InBreast datasets, respectively. They are at least $4 \%$ to $7 \%$ higher than the maximum accuracy produced when extracting local patterns using the LBP operator. The SVM classifier produced the highest classification accuracy by removing $6.5 \%$ of the local patterns when evaluated on the MIAS dataset $(\operatorname{LTP}(7,14)$ and $\operatorname{LTP}(7,16))$ where the MLP classifier $(\operatorname{LTP}(9,18))$ outperformed the other classifiers at $n=93.1 \%$ when tested on the InBreast dataset.

Figure 12 and 13 shows classification results using the LQP operator for the MIAS and InBreast dataset, respectively. Overall, it can be observed that LQP produced better classification results in comparison to LBP and LTP operators. The majority of the maximum accuracies are in a range between $74 \%$ to $79 \%$ which indicates that local patterns extracted by the LQP operator are more discriminant in comparison to the previous two operators. $\operatorname{LQP}(9,18)$ and $\operatorname{LQP}(9,20)$ produced a maximum accuracy of $82.1 \%$ with the best average accuracy of $78.6 \%$ at $n=90$ on the MIAS dataset. However, when evaluated on the InBreast dataset the maximum accuracy of $80.1 \%$ is achieved by $L Q P(9,18)$ at $n=98$ and the best average accuracy is achieved by $\operatorname{LQP}(9,20)$. Moreover, it can also be observed that the SVM classifier produced an average maximum accuracy over $80 \%$ regardless of the values of $P$ and $R$ when evaluated on the MIAS dataset. However, the MLP classifier 


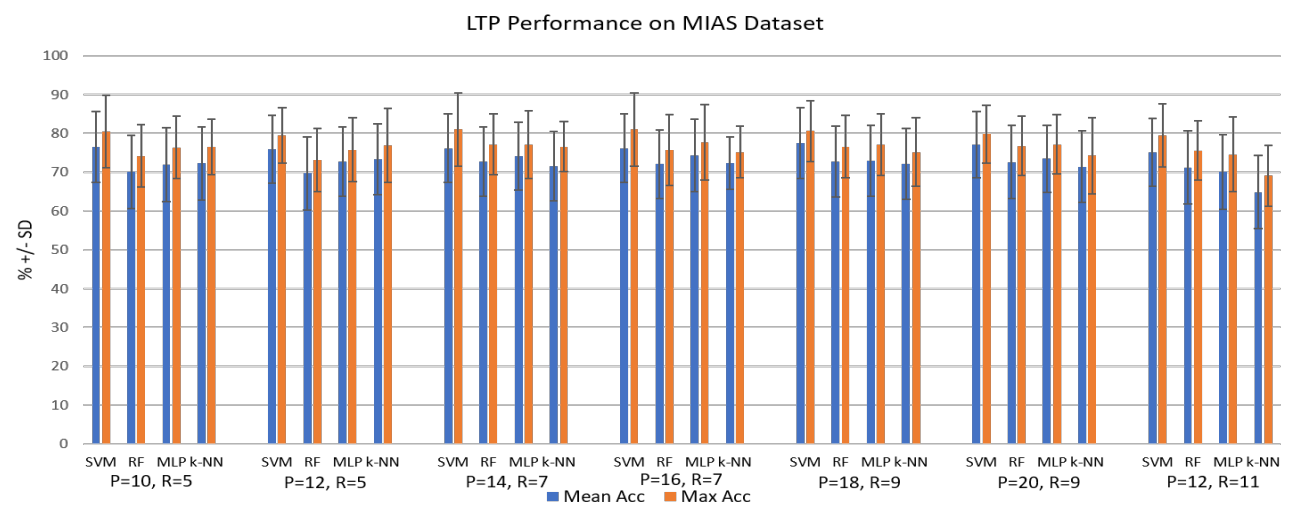

Figure 10: LTP performance on MIAS dataset

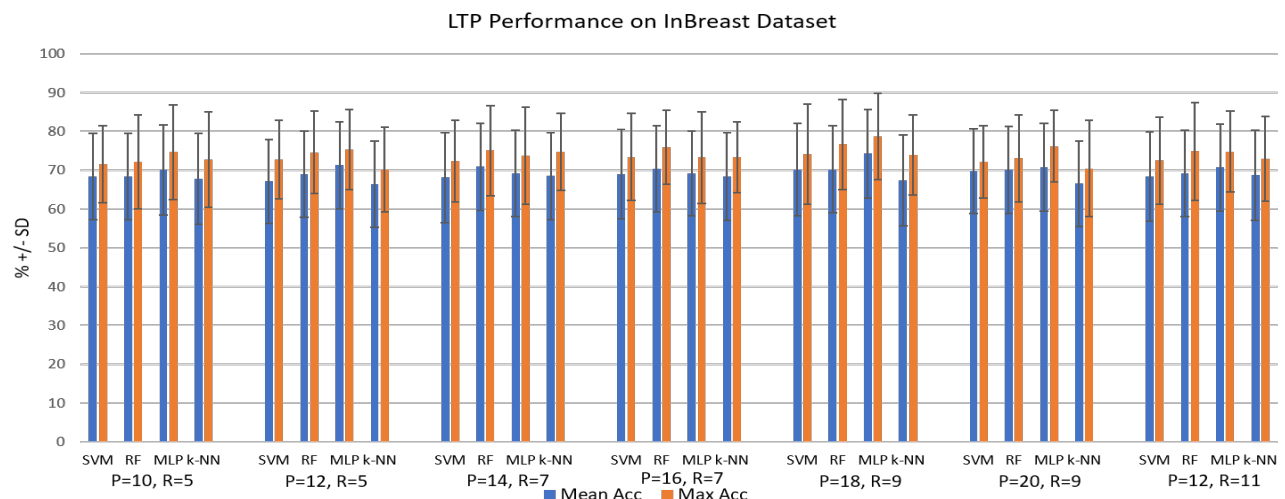

Figure 11: LTP performance on InBreast dataset

tends to produce better results when tested on the InBreast dataset with the best average $A c c=75.5 \%$ which is $1 \%$ better than the SVM classifier.

Table 14 and 15 shows quantitative results using our proposed LSP operator when evaluated on the MIAS and InBreast dataset, respectively. The majority of the classification accuracies fall in the range $75 \%$ to $80 \%$ which is slightly better compared to the results produced by the LQP operator. Experimental results show that the LSP operator produced a maximum accuracy of $83.3 \%$ (which outperforms the LBP (73.8\%), LTP (81\%) and LQP $(82.1 \%)$ operators) on the MIAS dataset. The best average accuracy of $81.6 \%$ is achieved by $\operatorname{LSP}(9,18)$ at $n=99.1$ which is at least $2.8 \%$ higher than the 


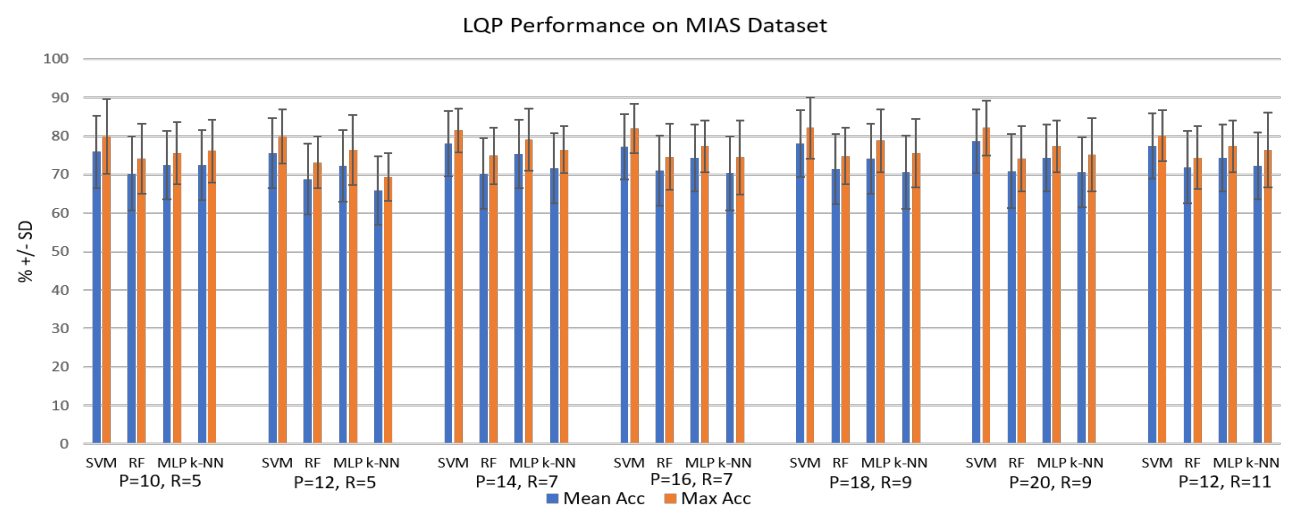

Figure 12: LQP performance on MIAS dataset

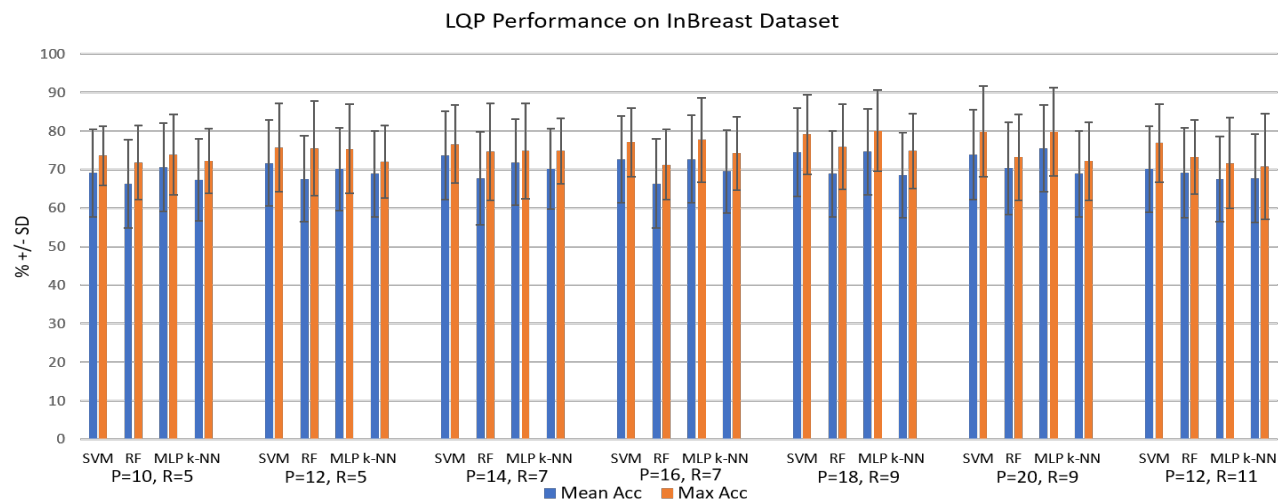

Figure 13: LQP performance on InBreast dataset

best average Acc produced by LQP and LTP. The LSP operator achieved maximum $80.5 \%$ classification accuracy at $n=95.5$ using the SVM classifier whereas the highest accuracy achieved by RF, $k$-NN and MLP are 77.9 $(\operatorname{LSP}(11,22)), 79.7 \%(\operatorname{LSP}(9,20))$ and $79.8(\operatorname{LSP}(7,16))$, respectively. Regarding the best average accuracy across different numbers of dominant patterns $\operatorname{LSP}(9,20)$ produced $77.1 \%$ using the SVM classifier. RF, $k$-NN and MLP produced $70.9 \%, 73.3 \%$ and $73.7 \%$, respectively when $\operatorname{LSP}(9,20)$ is employed. Overall, the LSP operator produced more discriminant local patterns in separating the BI-RADS classes in both MIAS and InBreast datasets. We will discuss this further in the subsequent subsections. 


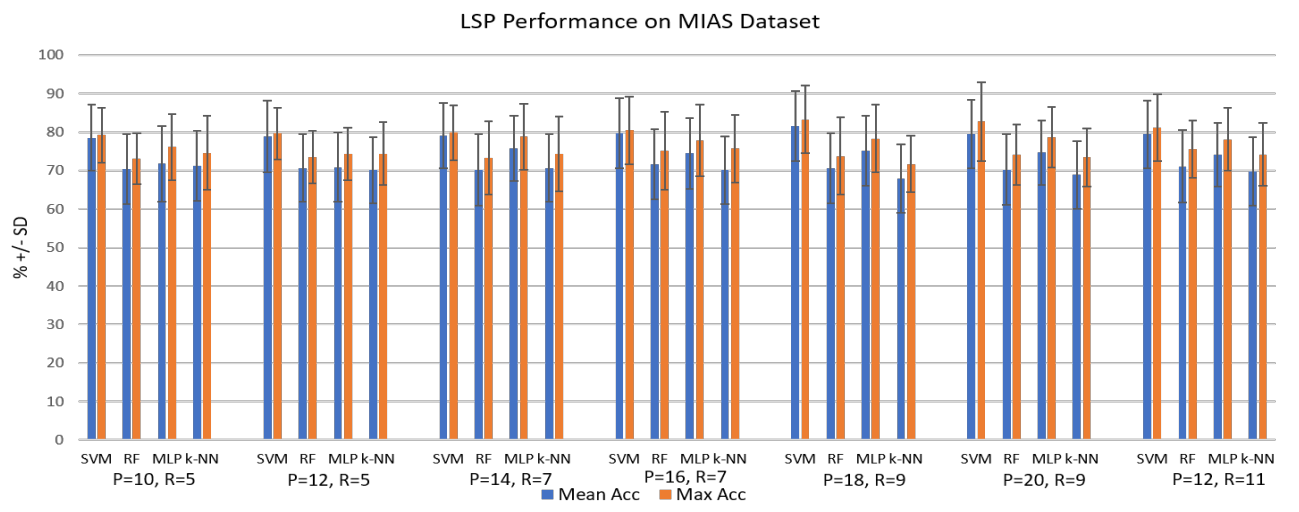

Figure 14: LSP performance on MIAS dataset

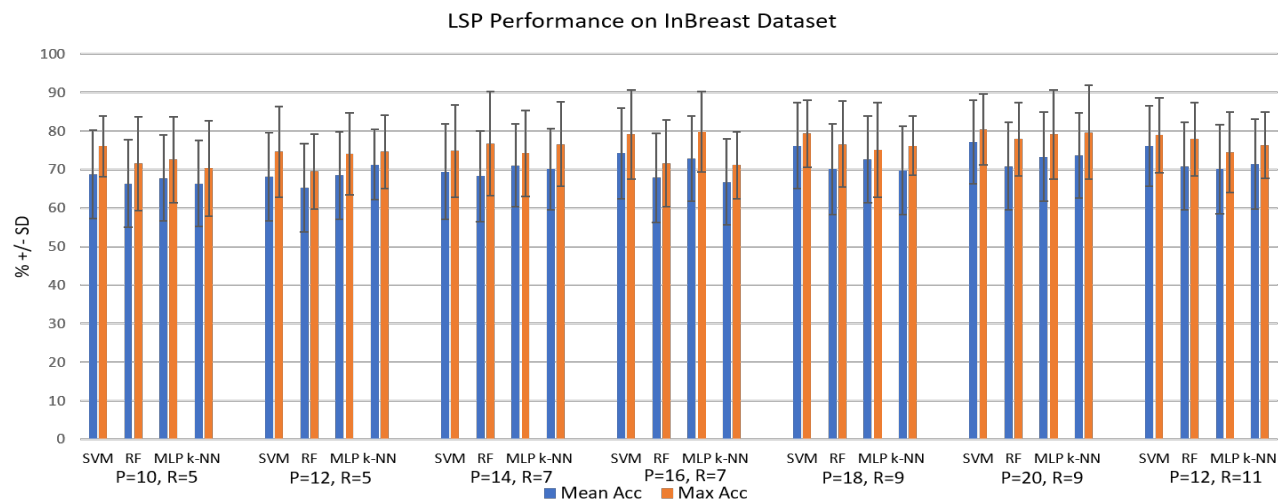

Figure 15: LSP performance on InBreast dataset

\subsection{Maximum and Average Performance Comparison Between Operators}

This section summarises the maximum and average results produced by the operators across different classifiers based on the MIAS and InBreast datasets. For the MIAS dataset, Figures 16 and 17 show performance comparisons between LBP, LTP, LQP and LSP operators using different classification approaches. In terms of maximum accuracy, it can be observed that LSP $(A c c=83.3 \%$ ) outperformed the other texture operators (LBP, LTP and LQP) when using SVM, RF and MLP as classification approaches. However, when the $k$-NN classifier is employed, the maximum classification accuracy is $76.9 \%$ using LTP which is $0.7 \%$ and $1.2 \%$ better than the LQP 
and LSP operators, respectively. Regarding the average accuracies across different numbers of dominant patterns, both the SVM and MLP classifiers produced higher results when using LQP and LSP features. For example, the LSP operator produced $81.6 \%$ and $75.8 \%$ accuracies for SVM and MLP classifiers, respectively compared to $78.6 \%$ (SVM) and $75.4 \%$ (MLP) when classification was performed based on local patterns extracted using the LQP operator. In contrast, classification accuracy is at least $0.9 \%$ better for local patterns using LTP when RF and $k$-NN classifiers are used compared to LQP and LSP. In both Figures 16 and 17, the LBP operator produced the lowest accuracy results regardless to classification approach which clearly shows that a channel encoding technique is necessary to capture more discriminant features and hence improve the classification results.

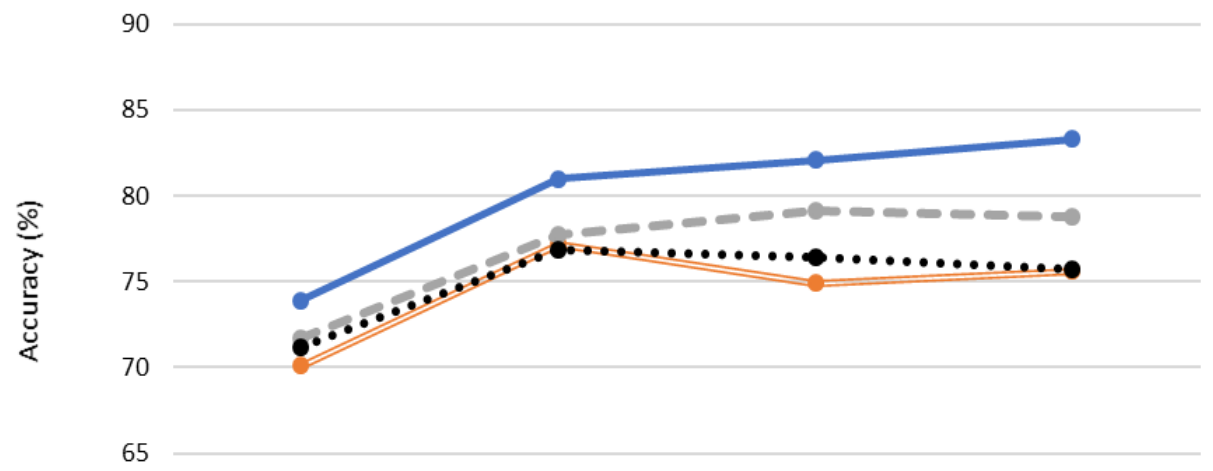

\begin{tabular}{|c|c|c|c|c|}
\hline 00 & LBP & LTP & LQP & LSP \\
\hline- SVM & 73.9 & 81 & 82.1 & 83.3 \\
\hline$\Longrightarrow \mathrm{RF}$ & 70.1 & 77.2 & 74.9 & 75.6 \\
\hline$-\mathrm{C}-\mathrm{MLP}$ & 71.7 & 77.7 & 79.1 & 78.8 \\
\hline$\bullet \bullet \bullet \bullet K N N$ & 71.2 & 76.9 & 76.4 & 75.7 \\
\hline
\end{tabular}

Figure 16: Comparison of maximum accuracies achieved by each operator across different classifiers when evaluated on the MIAS dataset.

For the InBreast dataset, Figures 18 and 19 show performance comparisons between all operators using different classifiers. Regarding maximum accuracy, the LSP operator achieved $A c c=80.5 \%$ using the SVM classifier and outperformed the LBP, LTP and LQP operators by $6.7 \%, 6.4 \%$, and $0.6 \%$, respectively. An improvement also can be observed for the $k$-NN classifier as the LSP operator produced $79.7 \%$ compared to the other operators 


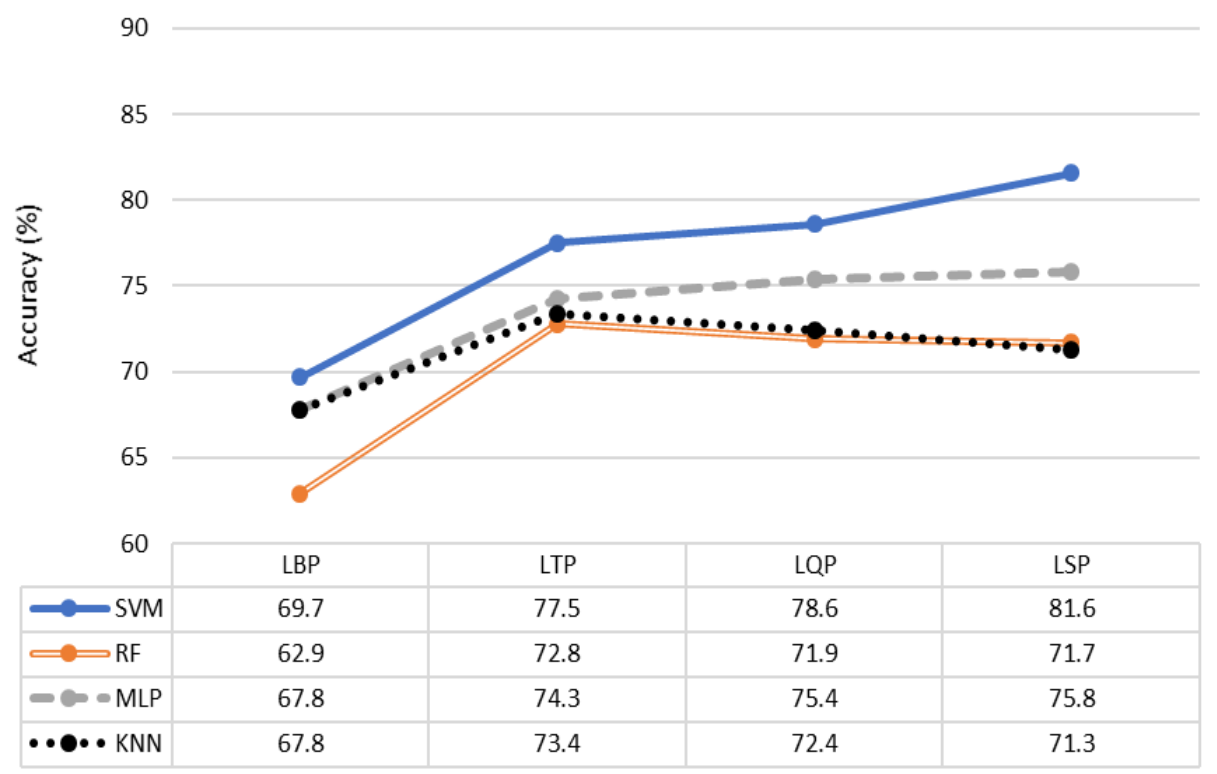

Figure 17: Comparison of average accuracies across different numbers of dominant patterns achieved by each operator across different classifiers when evaluated on the MIAS dataset.

which produced Acc $<75 \%$. For the RF classifier, the LSP operator produced $77.9 \%$, at least $1.2 \%$ higher compared to the other operators. The LQP operator produced a maximum accuracy of $80.1 \%$ which is $0.3 \%$ higher than the LSP operator when using the MLP classifier. Once again it can be observed that other operators always outperform the classification results of the LBP operator regardless of classifiers. In terms of average accuracies, the LSP operator once again outperforms the other operators when the SVM classifier is employed with the best average accuracy of $77.1 \%$. Similar trends can be seen when using the $k$-NN classifier where the LSP features produced the best average accuracy of $73.8 \%$ compared to $64.6 \%, 68.7 \%$ and $70.2 \%$ produced by the LBP, LTP and LQP features, respectively. The LSP and LTP operators produced similar results of $70.9 \%$ when the RF classifier is employed which is $0.6 \%$ higher than the LQP operator. The LSP operator produced the best average accuracy of $73.3 \%$ using the MLP classifier which is slightly lower compared to LQP and LTP operators with $75.5 \%$ and $74.3 \%$ accuracy, respectively. Once again experimental results suggest that the LSP operator extracts more discriminant local features and performs better when the SVM classifier is employed. 


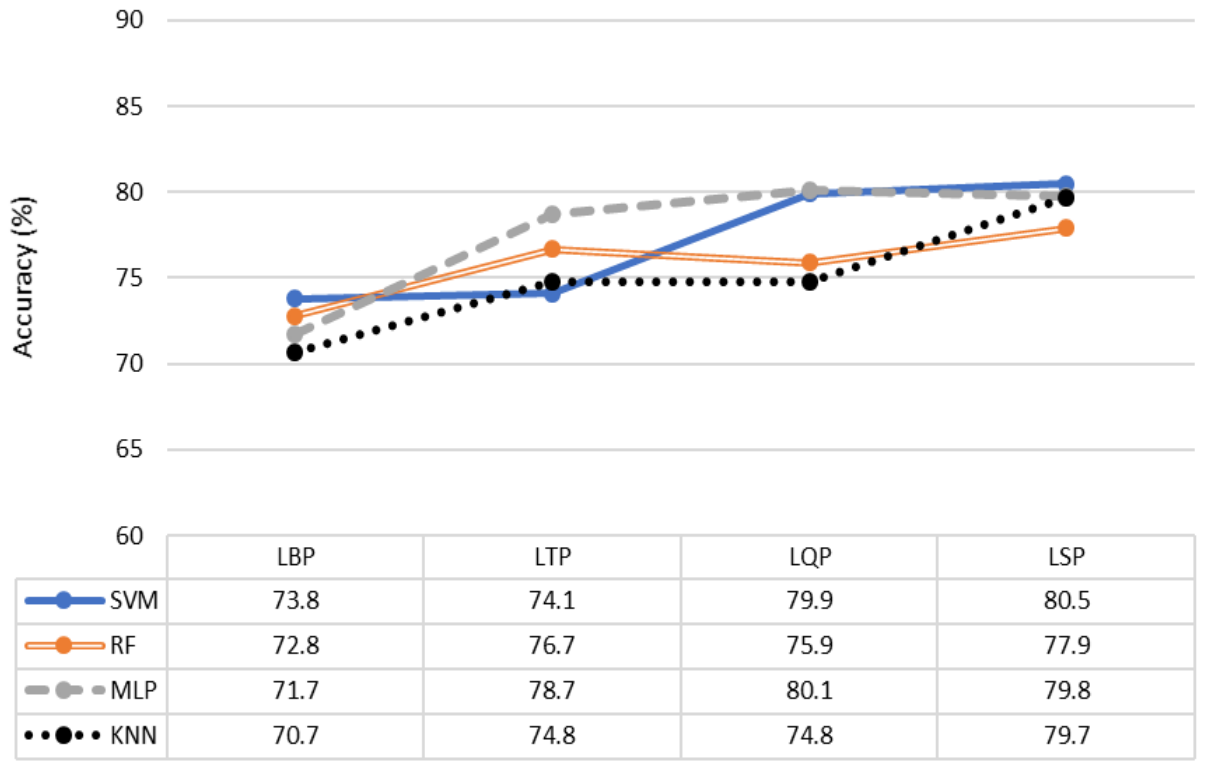

Figure 18: Comparison of maximum accuracies achieved by each operator across different classifiers when evaluated on the InBreast dataset.

\section{Discussion}

In this section we will discuss (i) the effects on accuracy when different numbers of dominant patterns are used in the classification, (ii) the effects of the radius $(R)$ and number of neighbours $(P)$ in the local patterns discriminatory levels; (iii) explanations on why multichannel local patterns produced more discriminant features in comparison to the original LBP operator, in (iv) extending to 9 and 11- encoding systems, (v) discussion of the existing studies in the literature, (vi) statistical analysis and (vii) future work.

\subsection{Effects of Different Numbers of Dominant Patterns (n)}

To investigate the effects of $n$ on the classification accuracy for all operators described in this study we tested 60 different values from 90 to 99.9 (e.g., $n \in\{90,90.1,90.3,90.5, \ldots, 99.9\}$ ). In other words, we investigated the variation of classification accuracy by including local patterns which have a frequency of minimum $0.1 \%$ to $10 \%$ in the histogram feature (e.g. local patterns with a relative frequency of less than $0.1 \%$ to $10 \%$ are removed, resulting to a shorter histogram feature). Figure 20 shows the effects of $n$ 


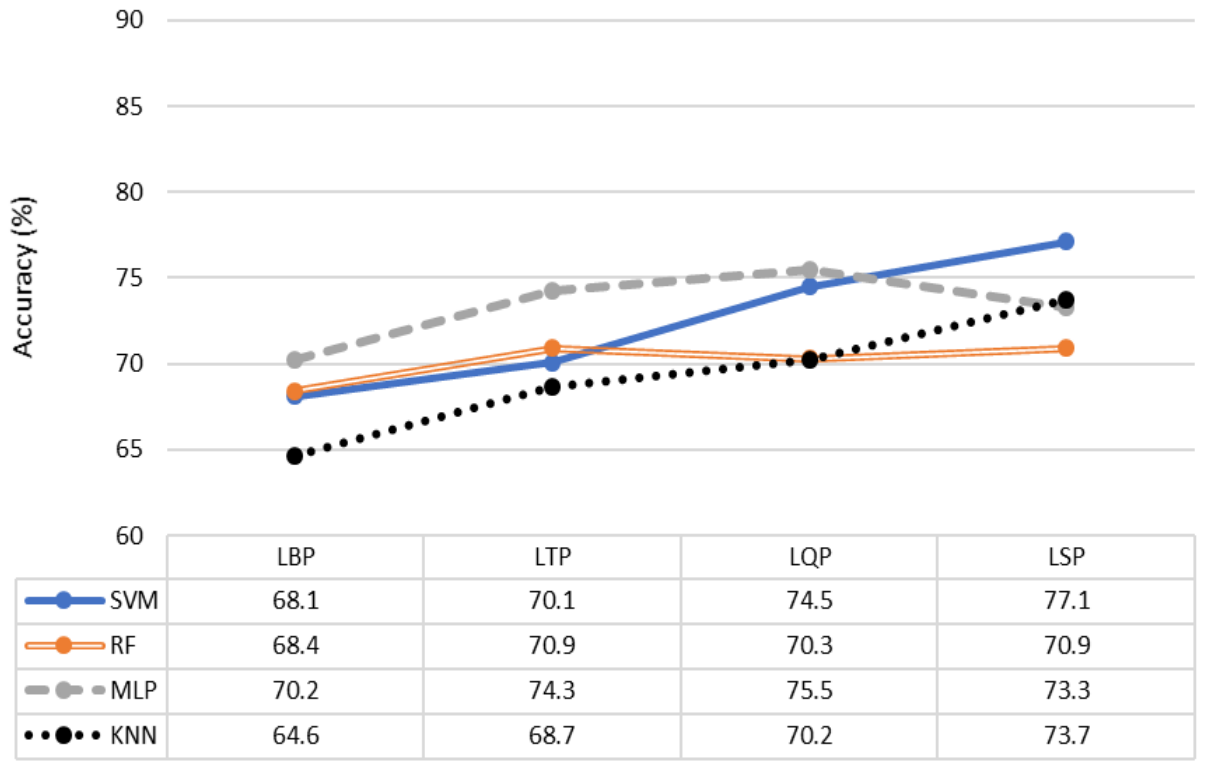

Figure 19: Comparison of average accuracies across different numbers of dominant patterns achieved by each operator across different classifiers when evaluated on the InBreast dataset.

on classification accuracy for LBP, LTP, LQP and LSP operators. In this experiment, we employed the SVM classifier as it produced better performance compared to the MLP, RF and $k$-NN classifiers. It can be observed that $n$ plays an important role in getting the best classification accuracy. For the LBP operator it can be observed that a large variation of classification accuracy between $63 \%$ to $74 \%$ occurs, with a $9.71 \%$ standard deviation. The LQP operator produced a standard deviation of $9.25 \%$ which is higher than the LTP operator of $9.04 \%$. The LSP operator produced a smaller standard deviation value of $9.04 \%$ (the same as LTP) which indicates that our proposed method does not only outperform the other operators on both datasets but also produced more consistent results.

\subsection{Effects of Radius (R) and Number of Neighbours (P)}

In this section we are interested to know to what extent $R$ and $P$ affect the overall classification accuracy. For this purpose, we investigated seven different combination of $R$ and $P$ and tested each of them with all operators. Figures 21 and 22 show average maximum accuracies for both datasets using 


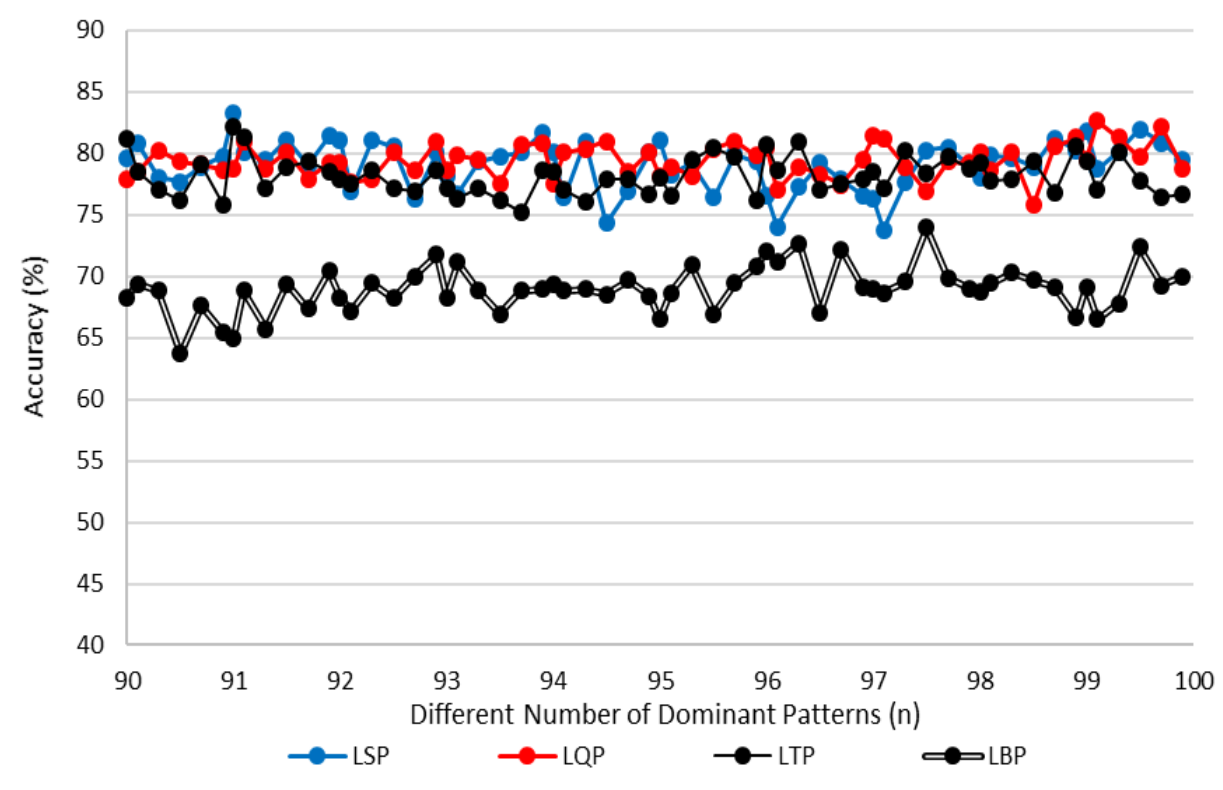

Figure 20: Effects of different numbers of dominant patterns $(n)$ across different operators $((\mathrm{P}, \mathrm{R})=(18,9))$ using SVM classifier on MIAS dataset.

the SVM classifier. For the LBP operator, local patterns extracted using smaller radii and less number of neighbours (e.g., $(5,10)$ and $(5,12))$ tend to produce better results whereas the LTP operator produced its highest average maximum accuracy at $(7,14)(A c c>78 \%)$. Similar results of $77.5 \%$ are obtained when the following parameters are employed: $(7,16),(9,18)$ and $(9,20)$. However, when evaluated on the InBreast dataset, the highest average maximum accuracy $(76 \%)$ is achieved at $(9,18)$ followed by parameters $(7,14)$ and $(7,16)$. For the LQP operator, the highest average maximum accuracy $(A c c>78 \%)$ is achieved at parameters $(7,14)$ followed by $(9,18)$ with only $0.5 \%$ difference. In the InBreast dataset, the LQP operator produced highest average maximum accuracy of $77.5 \%$ with parameters $(9,18)$. The LSP operator produced consistent average maximum accuracy when parameters $(7,14),(7,16),(9,18),(9,20)$ and $(11,22)$ are used which is around $77 \%$. Nevertheless, it produces at least $2.5 \%$ higher average maximum accuracy at $(9,20)$ compared to $(7,14),(7,16),(9,18)$ and $(11,22)$. These results indicate that when choosing the values for $P$ and $R$, the following values $(7,16)$, $(9,18),(9,20)$ are a good starting point.The LSP operator produced its best 
results at $(9,20)$ using the SVM classification approach.

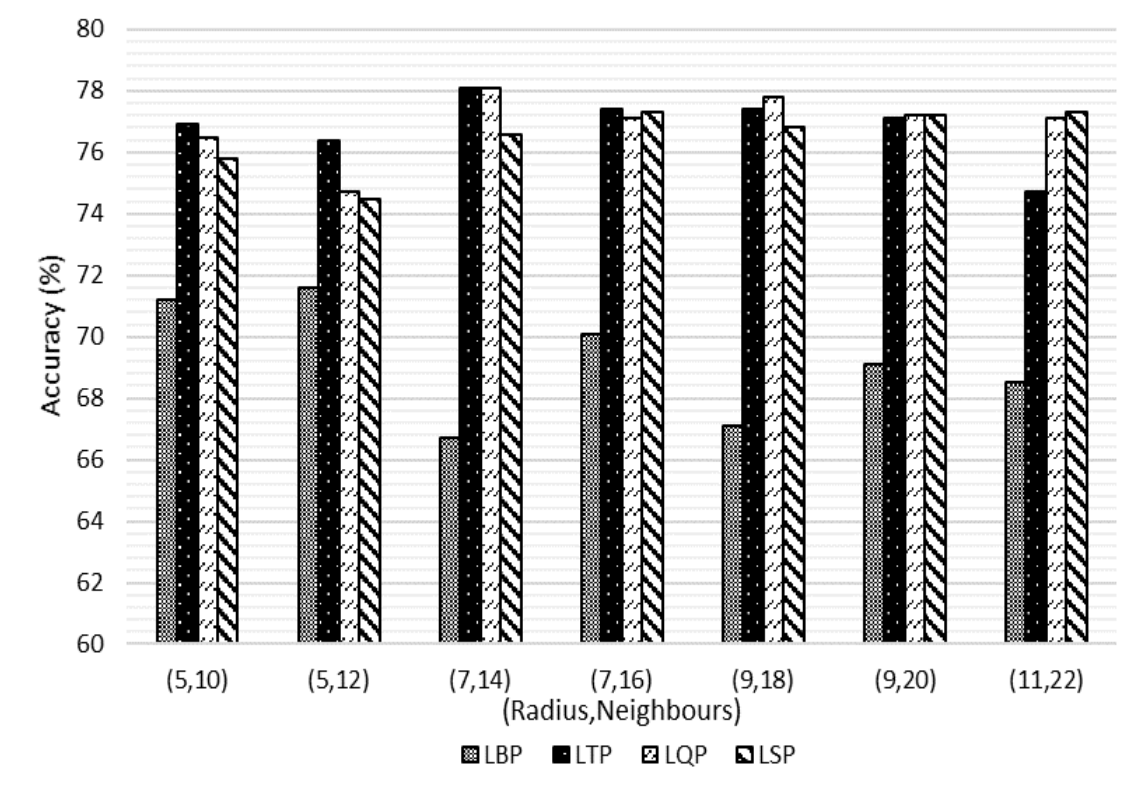

Figure 21: Average maximum accuracy (MIAS dataset) across different classifiers based on different operators and their associated parameters.

\subsection{Why do multichannel local patterns work?}

To answer this question we conducted several experiments by performing channel based classifications on the MIAS dataset. In the case of the LTP operator (three channels with two binary patterns), firstly we perform individual classification based on features extracted from the first binary patterns and secondly we concatenate features extracted from two or more channels. Table 3 shows our experimental results for the LTP, LQP and LSP operators. Note that the following parameters are used based on their best performance using the SVM classifier (see Figures 12, 13 and 14): $\operatorname{LTP}(7,16)$ with $n=93.5, \operatorname{LBP}(9,18)$ with $n=99.9$ and $\operatorname{LSP}(9,18)$ with $n=99.1$.

It can be observed that classification based on features extracted from a single channel alone is insufficient. For the LTP operator, each channel produced just over $73 \%$ but concatenating local patterns from all channels produced $81 \%$, yielding over $7 \%$ improvement. A similar case occurred for the LQP operator where binary patterns from a single channel produced $A c c<70 \%$. However, combining binary patterns from the first three 


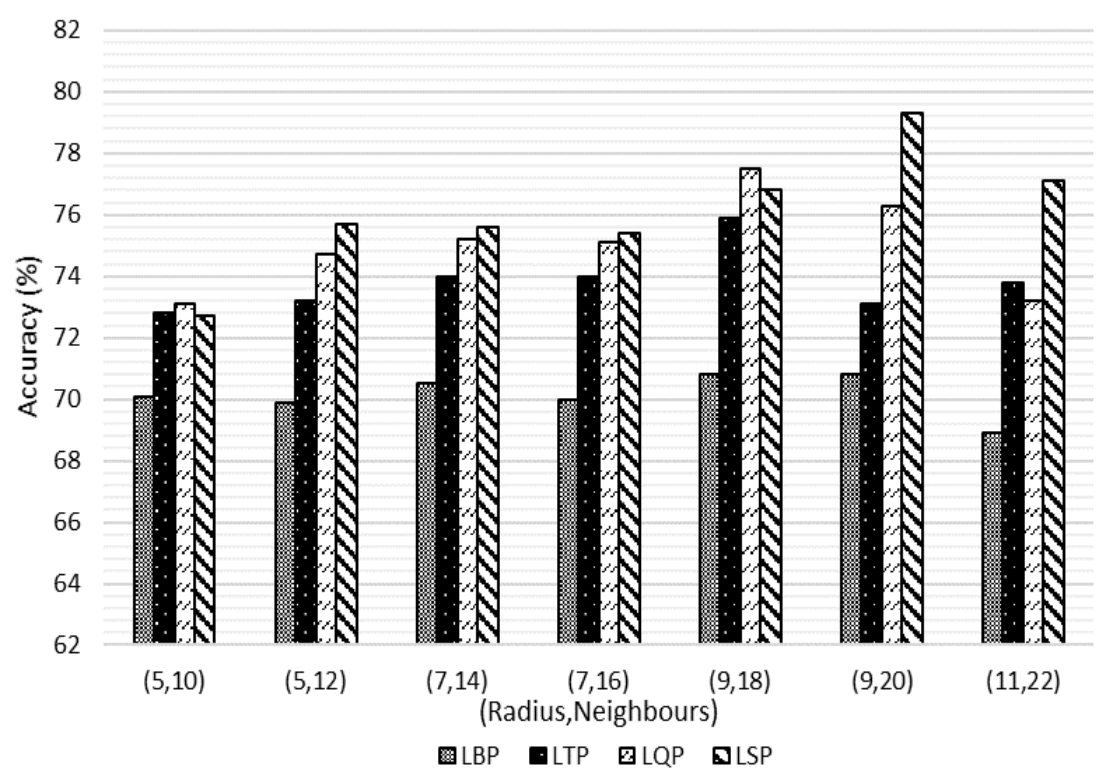

Figure 22: Average maximum accuracy (InBreast dataset) across different classifiers based on different operators and their associated parameters.

channels $(C h 1 \oplus C h 2 \oplus C h 3)$ yields a significant improvement $76.4 \%$ and concatenating all local patterns from all channels produced a maximum accuracy of $82.1 \%$. For our proposed operator, $C h 2$ and $C h 5$ produced classification accuracy under $60 \%$ and $C h 1, C h 3$ and $C h 4$ produced accuracy close to $70 \%$. Concatenating all features from all channels resulted in $A c c=83.3 \%$ however removing a less informative channel (e.g., $C h 2$ ) by considering $C h 1 \oplus C h 3 \oplus C h 4 \oplus C h 5 \oplus C h 6$, the classification accuracy improved to $83.8 \%$.

Based on these experiments we found that encoding an image by dividing it into several channels captures more textural details which is difficult to capture with the original LBP operator. This can be clearly seen in Figures 8 and 9 where most accuracies achieved by the LBP operator are under $70 \%$ whereas the other operators consistently achieved above $75 \%$ accuracy. This also indicates that the LQP and LTP operators capture more details of the Corpus Mammae region in comparison to the LBP operator. Similarly, the LSP operator captures even more details compared to LQP and LTP operators. However, a higher order encoding system such as dividing into nine or eleven channels does not necessarily improve the classification accuracy 


\begin{tabular}{|c|c|c|}
\hline Operators & Channel $(C h)$ & Channels Concatenation $(\oplus)$ \\
\hline LTP & $C h 1=73.3 \pm 8.6$ & $C h 1 \oplus C h 2=81.95$ \\
& $C h 2=74.1 \pm 9.1$ & $C h 1 \oplus C h 2=69.3 \pm 8.3$ \\
\hline \multirow{4}{*}{ LQP } & $C h 1=59.8 \pm 10.6$ & $C h 1 \oplus C h 2 \oplus C h 3=76.4 \pm 8.1$ \\
& $C h 2=65.9 \pm 9.6$ & $C h 1 \oplus C h 2 \oplus C h 3 \oplus C h 4=82.1 \pm 7.1$ \\
& $C h 3=68.9 \pm 11.5$ & \\
\hline \multirow{5}{*}{ LSP } & $C h 4=66.5 \pm 9.9$ & $C h 1 \oplus C h 2=74.3 \pm 7.9$ \\
& $C h 2=52.6 \pm 11.6$ & $C h 1 \oplus C h 2 \oplus C h 3 \oplus C h 4=70.1 \pm 10.1$ \\
& $C h 3=70.1 \pm 8.8$ & $C h 1 \oplus C h 2 \oplus C h 3 \oplus C h 4 \oplus C h 5=77.7 \pm 9.1$ \\
& $C h 5=59.3 \pm 11.3$ & $C h 1 \oplus C h 3 \oplus C h 4 \oplus C h 6=80.7 \pm 10.1$ \\
& $C h 4=65.7 \pm 12.8$ & $C h 1 \oplus C h 3 \oplus C h 4 \oplus C h 5 \oplus C h 6=83.8 \pm 9.8$ \\
& & $C h 1 \oplus C h 2 \oplus C h 3 \oplus C h 4 \oplus C h 5 \oplus C h 6=83.3 \pm 8.8$ \\
\hline
\end{tabular}

Table 3: Classification results (\%) based on binary patterns extracted from individual channel (second column) and concatenated binary patterns from two or more channels

because it increases the number of features yielding more complex decision boundaries. An early indication can be observed based on our experiment results in Table 3 where best accuracy can be achieved by the LSP operator when excluding local patterns from $C h 2$. This reduces the number of features yielding a less complex decision boundaries in a feature space, hence boosting the overall performance of the method.

A channel encoding system also extracts a set of 'weak' features which produce satisfactory results on its own, but when combined they provide good results (similar to ensemble classifiers such as the Random Forests). For example, in Table 3 combining two or more local patterns from different channels always produce better results. This is due to each channel containing unique information which is not available in the other channels and they complement each other when combined. For example, individual classification in LQP produce under $70 \%$ accuracy but improve to over $76 \%$ and $82 \%$ when three and four channels are combined, respectively. Simi- 
larly, all channels from the LSP operator are unable to provide sufficient information of the Corpus Mammae region individually but when combining $C h 1 \oplus C h 3 \oplus C h 4 \oplus C h 5 \oplus C h 6$ we achieved $83.8 \%$ accuracy.

Moreover, the threshold values which are determined automatically enable the operator to capture robust texture information as pixel information is encoded according to the local threshold values rather than a global threshold value as in the LTP and LQP operators. Local threshold values enable the LSP operator to capture textural information based on the topology and intensity distribution of the image and hence are more discriminant and tolerant to noise. In contrast, a global threshold value does not consider intensity distribution of a region. As a result, it may affect the discriminatory level of the local patterns.

\subsection{Extending to 9-encoding and 11-encoding systems}

To further evaluate the performance of different encoding systems, we conducted several experiments by extending the LSP to 9- and 11-encoding systems both on the MIAS and InBreast datasets. Figures 23, 24, 25 and 26 show that the performance is decreasing as we use 9- and 11-encoding systems.

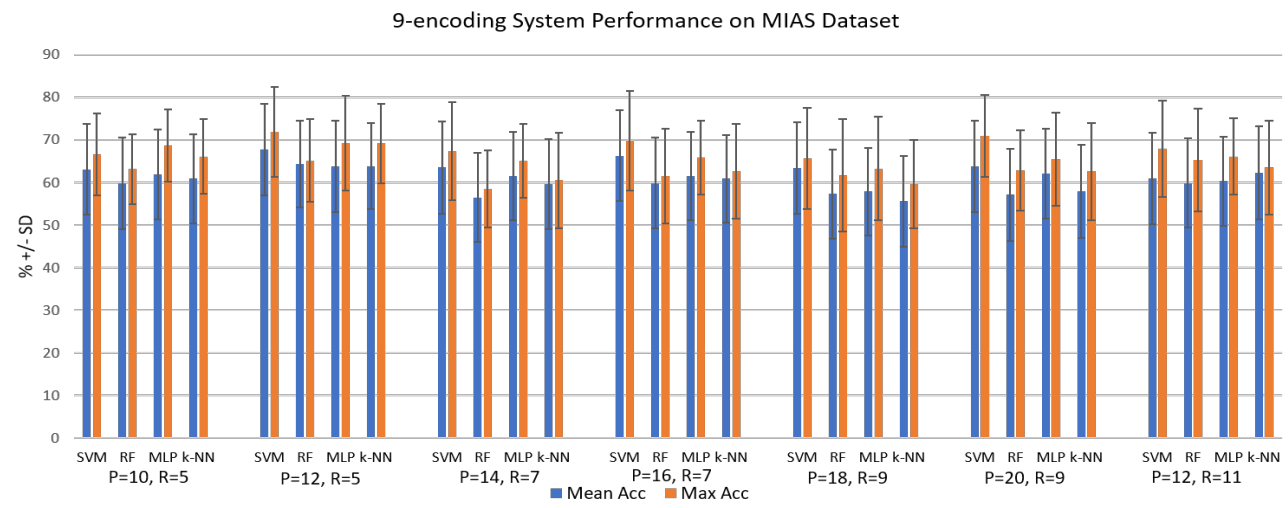

Figure 23: 9-encoding system performance on MIAS dataset

Experimental results suggest that the 7-encoding system the LSP achieved the best performance and eventually decreased to between $55 \%$ to $63 \%$. From our own observation, the main reason for this is due to higher encoding systems (e.g. 9-encoding) producing very sparse features (containing many 
zeros). This is similar when performing the 11-encoding system (in fact features are even sparser). The main problem with sparse features is they are most likely being ignored by most classifiers particularly tree-based machine learning algorithms. Secondly, in most cases zeros are less informative making the features less meaningful and hence less discriminant. As a result, performance classification reduced significantly. In Figure 25 and 26 none of the maximum accuracies achieved was above $70 \%$ whereas the LBP operators achieved above this value in some cases.

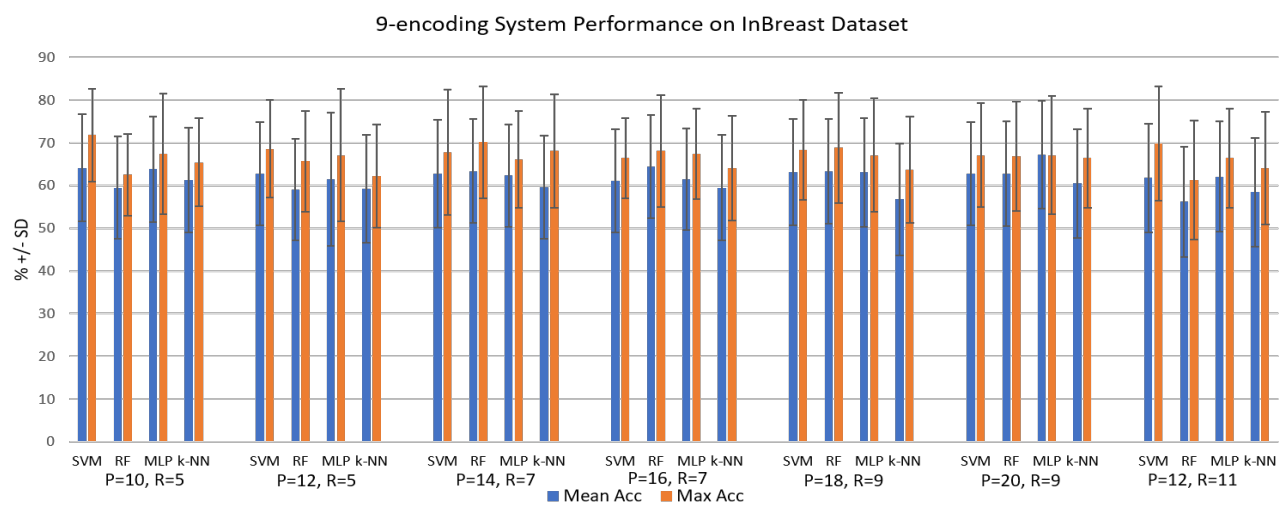

Figure 24: 9-encoding system performance on InBreast dataset

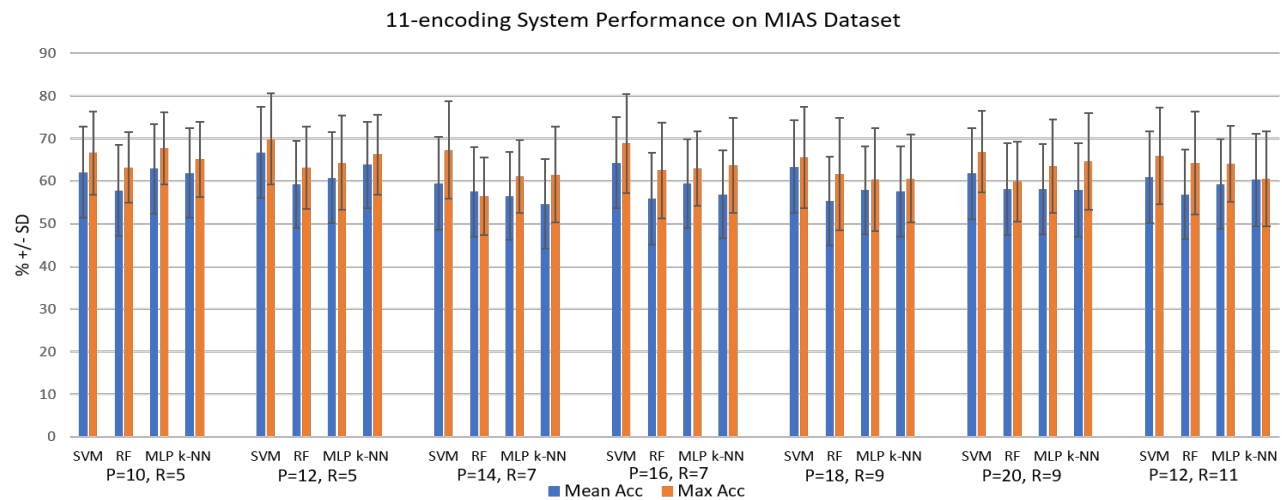

Figure 25: 11-encoding system performance on MIAS dataset 


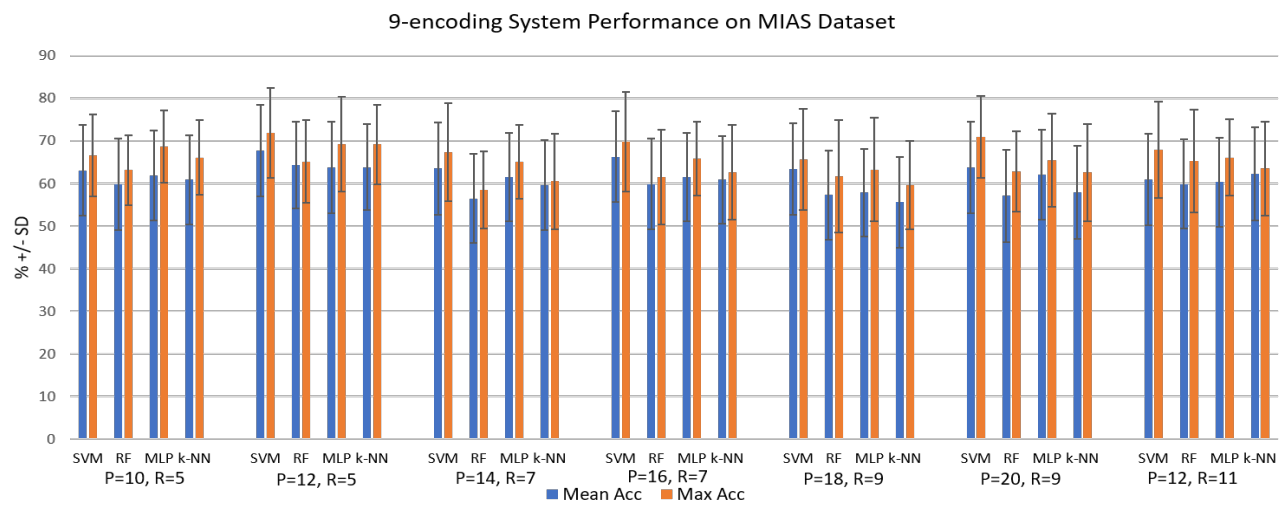

Figure 26: 11-encoding system performance on InBreast dataset

\subsection{Existing Methods in the Literature}

The main goal of our study is to improve the performance of binarybased local pattern feature extraction methods, namely Local Binary Pattern (LBP), Local Ternary Pattern (LTP) and Local Quinary Pattern (LQP). Our study does not intend to quantitatively compare the performance of the LSP operator against other feature extraction methods. There are many feature extraction methods in the literature, therefore quantitative comparison is extremely difficult. It should also be noted that quantitative comparison with the other breast density methods in the literature is because most studies combine features from different feature extraction algorithms such as first-order statistical features, second-order statistical features, morphological features, features of Gabor filters, edge information, etc.

Many breast density classification methods have been developed over the last two decades. The best classification accuracy (based on BI-RADS classes (fourth edition)) reported in the literature is over $90 \%$ by the study by [14] followed by the study of [11] with $86 \%$ classification accuracy. However, these methods are computationally expensive due to different numbers of feature extraction techniques employed. Several techniques used to extract different types of features such as the first order statistical techniques to compute the local pixel intensity, the co-occurrence matrix to extract grey level distribution, an adaptive thresholding technique to extract the region's morphological information, Gabor filter to obtain edges information, etc. Also, their methods employed the Fuzzy C-Means clustering technique as a separate process to classify the breast tissue into different pixel intensities. Once again this 
process is time consuming especially when dealing with large images such as mammograms. In contrast, the LSP operator (with accuracy close to $84 \%$ ) extracts and processes all this information at once, hence reducing the computational complexity of the system.

Previously, [20] used the LTP operator to extract local information and achieved a promising result of $82.33 \%$ accuracy. However, this method suffers from having to deal with a large number of features (over one thousand) due to the multi-orientation approach (e.g., ten histograms from ten orientations concatenated). Our recent study, [21] reported the best accuracy of over $86 \%$ using multi-orientation LQP operator and combining other local patterns extracted using on ellipse neighbourhood. Nevertheless, this method contains several separate stages (e.g. extraction using different neighbourhood topologies (circle and ellipse), different orientations of resolutions which can be tedious whereas the LSP operator uses only one neighbourhood topology (e.g. a circle) with a single resolution. Other studies in the literature such as $[11,12,13,14,15,18,19,22,23]$ reported accuracies under $80 \%$. However, note that the purpose of our study is not to develop a breast density classification method that can necessarily outperform the other methods in the literature but to study the effects of various channel encoding techniques in the original LBP operator which have lead us to a more robust technique called the LSP operator, improving the performance of the LBP, LTP and LQP operators.

\subsection{Statistical Analysis}

We performed statistical analysis to investigate whether there is a significant difference/improvement at $p \leq 0.05$ between the best results achieved by the LSP operator and the other operators discussed in this study on both datasets. For this purpose, we compared the best maximum (Max) and average (Avg) accuracies produced by the LSP with the best results of LBP, LTP and LQP operators. The $p$-value was computed using a $t$-test comparing each result of each operator with the best result achieved by the LSP operator according to their respective metric (e.g., Max Acc or Avg Acc). The size of population for each dataset are 103 (206 images) and 161 (322 images) for the InBreast and MIAS dataset, respectively.

Table 4 shows the $p$-values between the best accuracies achieved by the LSP operator and the other operators for both datasets. In terms of maximum accuracy, there is a significant improvement at $p \leq 0.05$ when using the LSP features in classifying breast density on the MIAS $(p=0.0015)$ and 


\begin{tabular}{|l|l|l|l|l|l|}
\hline Dataset & Results & LBP & LTP & LQP & LSP \\
\hline \multirow{5}{*}{ MIAS } & Max Acc $(\%)$ & $73.8 \pm 10.6$ & $81 \pm 9.5$ & $82.1 \pm 7.1$ & $83.3 \pm 8.8$ \\
& $(p$ value $)$ & $(0.0015)$ & $(0.2260)$ & $(0.4019)$ & - \\
\cline { 2 - 6 } & Avg $A c c(\%)$ & $69.7 \pm 9.7$ & $77.5 \pm 9.2$ & $78.6 \pm 8.3$ & $81.6 \pm 9.1$ \\
& $(p$ value $)$ & $(0.00001)$ & $(0.0428)$ & $(0.0433)$ & - \\
\hline \multirow{5}{*}{ InBreast } & Max Acc $(\%)$ & $73.8 \pm 9.9$ & $78.7 \pm 11.2$ & $80.1 \pm 10.5$ & $80.5 \pm 9.2$ \\
& $(p$ value $)$ & $(0.0064)$ & $(0.2646)$ & $(0.4489)$ & - \\
\cline { 2 - 6 } & Avg $A c c(\%)$ & $70.2 \pm 11.7$ & $74.3 \pm 11.4$ & $75.5 \pm 11.3$ & $77.1 \pm 10.9$ \\
& $(p$ value $)$ & $(0.0007)$ & $(0.0137)$ & $(0.2151)$ & - \\
\hline
\end{tabular}

Table 4: Classification results (\%) and $p$-values between the best accuracies achieved by the LSP operator and the other operators discussed in this study.

InBreast $(p=0.0007)$ datasets. Nevertheless, the best maximum results of the LSP operator are not statistically significant at $p \leq 0.05$ in comparison with the best accuracies achieved by the LTP and LQP operators. The $p$-values are 0.2260 and 0.4019 for the LTP and LQP operators, respectively when evaluated on the MIAS dataset which are similar when tested on the InBreast dataset, $p=0.2646$ and $p=0.4489$ for the LTP and LQP operators, respectively. The results are statistically significant at $p \leq 0.05$ on the MIAS dataset when comparing the best average accuracy produced by the LSP features $(A c c=81.6 \%)$ with the other features with $p$-values are 0.00001, 0.0428 and 0.0433 for LBP, LTP and LQP respectively. Similarly, when tested on the InBreast dataset the best average accuracy produced by the LSP operator is significantly better in comparison to the results of LBP $(p=0.0007)$ and LTP $(p=0.0137)$ operators. However, the best average accuracy of LSP is not significant $(p=0.2151)$ compared to the result of the LQP operator.

\subsection{Limitations of our study}

We highlight the following limitations of our study:

1. The sample dataset used to optimise the classifiers' parameters was taken from the MIAS dataset which only 8-bit contrast. This means the optimised parameters and testing results might be different when 
testing with real clinical data (12 to 14 bits contrast). The reasons we chose the MIAS dataset rather than the InBreast dataset when optimising the classifiers' parameters are (a) it has larger number of images/cases hence giving us a larger number of test cases and (b) the number of cases for each class is more representative compared to the number of cases for each class in the InBreast dataset. For example, the number images of BI-RADS IV is 14 which is extremely small. Including cases in our dataset for parameter optimization, will make our testing dataset extremely imbalanced. Although in this study we used images from the MIAS dataset for parameter optimization, in a real clinical environment this procedure can be easily changed/adapted by using a sub-sample from the new dataset.

2. The MIAS dataset is a somewhat old database and hence does not represent the actual contrast representation of the latest mammograms. We included the MIAS dataset in our study due to (a) it is the most used and compared dataset in the literature because of its availability whereas other datasets are not easily accessible, (b) acquiring mammogram datasets is very difficult especially the ones with BI-RADS (version 4) classification and (c) other datasets do not classify each breast image based on BI-RADS density but are classified based on a small square patch based on tissue types such as 'fatty', 'glandular' or 'breast tissue'.

3. The parameters $P, R$ and $n$ were tested/selected empirically. Nevertheless, our testing strategy is based on the previous studies of Ojala et al. [32], Tan and Triggs [33], Nanni et al. [34]. To the best of our knowledge this a common approach used in the literature especially for parameters $P$ and $R$. For parameter $n$, we tested 60 different values from $n=90$ to $n=99.9$ with 0.2 interval (e.g. 90, 90.1, 90.3, 90.5, etc). This should be sufficient to investigate the performance variations across different $n$ values.

4. The number of cases used in the development of parameter optimization is small. However, we prefer to optimise the number of images for testing purposes and therefore, we limit the number of cases for parameter optimisation. It is more important to test the model performance on a larger size of dataset rather than on a smaller dataset.

\subsection{Future Work}

For future work we plan to consider the following: 
1. Since multichannel based operators such as LTP, LQP and LSP require the number of dominant patterns $(n)$ to obtain an optimum classification accuracy, we plan to develop a statistical method that can automatically determine an optimum value of $n$.

2. We also interested to investigate the performance of LBP's two-channel variants such as M-ELBP, ELBP, MRELBP, STLBP and CLBP when multichanel based operators are being applied.

3. As indicated in Table 3, combining all local patterns from all channels does not necessarily produce optimal accuracy. For example, the LSP operator achieved its highest accuracy when excluding $C h 2$. Therefore, we plan to develop a method that can automatically determine the most informative local patterns from each channel and in the classification phase only combine the most discriminant features. This can be done by measuring mutual information among the histogram features and combining the ones with the least overlapping information.

4. We plan to develop a deep learning based network to extract local features of the Corpus Mammae region and combine them with features extracted with the LQP or LSP operators.

\section{Summary and Conclusions}

In this paper, we studied the effects of various channel encoding techniques in local pattern extraction which have led us to a more robust technique called the LSP operator. We investigated the following aspects in our study:

1. We made comparisons between the LBP, LTP, LQP and LSP operators based on MIAS and InBreast datasets. Experimental results suggest that the proposed texture operator outperformed the other operators on both datasets.

2. We investigated whether the choice of a classifier can influence the performance of the system. We employed four different classifiers and found that in many cases the SVM classifier outperforms the other classifiers. Local patterns extracted with the LTP operator tend to produce better results when the MLP or $k$-NN classifier is employed whereas the SVM classifier produced better accuracy using LQP and LSP features. 
3. We conducted experiments to investigate whether the operators discussed in this study have a particular preference regarding $R$ and $P$. We found that in many cases LBP features produced similar results of under $70 \%$ accuracy. However, the LSP features tend to produce better classification results when $\operatorname{LTP}(7,14)$ and $\operatorname{LTP}(9,18)$ are used. On the other hand, both LQP and LSP operators tend to produce consistent results when $R \in\{7,9\}$ and $P \in\{16,18,20\}$ are used.

4. We studied the effects of selecting different numbers of dominant patterns and experimental results show that the value of $n$ plays an important role in obtaining optimal accuracy. The LSP and LTP operators produced variation accuracy of $9.04 \%$ compared to $9.25 \%$ and $9.71 \%$ for LQP and LBP, respectively.

5. We also studied channel based classification and concatenating local patterns from different channels. Experimental results suggest that each channel contains unique features which are not available in the other channels and combining these features yielded to better classification results. We also found that removing the most uninformative channel in LSP features improves the classification accuracy by $0.5 \%$.

6. We developed simple methods to automatically determine the threshold values in LSP by computing the $25^{\text {th }}$ percentile and $75^{\text {th }}$ percentile of the neighbouring pixels.

In conclusion, we have studied various channel encoding techniques in LBP, LTP and LQP operators where we found that multichannel local patterns are more robust in discriminating different classes of breast density. This study has led us to the development of a seven-encoding technique call LSP operator to capture more texture details within the Corpus Mamae region. We also introduced a simple method to automatically determine the threshold values in the LSP operator by computing the first order statistical values of the neighbourhood pixels. Experimental results show that the LSP features outperformed the LBP, LTP and LQP operators on both MIAS and InBreast datasets. The LSP features produced a maximum accuracy of $83.8 \%$ using the SVM classifier when combining local patterns from $C h 1$, $C h 3, C h 4, C h 5$ and $C h 6$ on the MIAS dataset. The LBP, LTP and LQP features achieved maximum accuracies of $73.8 \%, 81 \%$ and $82.1 \%$, respectively. Based on these experimental results, the proposed seven-encoding system approach (LSP) is shown to be a robust and more consistent texture operator. 


\section{Declaration of competing interest}

None Declared.

\section{Acknowledgment}

This research was undertaken as part of the Decision Support and Information Management System for Breast Cancer (DESIREE) project. The project has received funding from the European Union's Horizon 2020 research and innovation programme under grant agreement No 690238.

\section{References}

[1] American Cancer Society, Breast cancer facts and figures 2017/2018, 2018. Available at https://www.cancer.org/content/ dam/cancer-org/research/cancer-facts-and-statistics/ breast-cancer-facts-and-figures/breast-cancer-facts-and-figures-2017-2018. pdf Accessed: 2017-06-23.

[2] C. E. DeSantis, J. Ma, A. G. Sauer, L. A. Newman, A. Jemal, Breast cancer statistics, 2017, racial disparity in mortality by state., CA: A Cancer Journal for Clinicians 67 (2017) 439-448.

[3] Breast Cancer, UK, Breast cancer care. facts and statistics 2018, 2018. Available at https://www.breastcancercare.org.uk/ about-us/media/press-pack-breast-cancer-awareness-month/ facts-statistics Accessed: 2017-05-23.

[4] Breast Cancer, UK, Breast cancer symptoms, 2018. Available at https://www.cancerresearchuk.org/about-cancer/ breast-cancer/symptoms Accessed: 2017-06-26.

[5] Breast Cancer, U.S. breast cancer statistics, 2016. Available at http: //www.breastcancer.org/symptoms/understand_bc/statistics Accessed: 2017-06-28.

[6] Breastcancer.org, Dense breast, 2018. Available at https://www. breastcancer.org/risk/factors/dense_breasts Accessed: 2017-0628. 
[7] G. Litjens, T. Kooi, B. E. Bejnordi, A. A. A. Setio, F. Ciompi, M. Ghafoorian, J. A. van der Laak, B. van Ginneken, C. I. Sánchez, A survey on deep learning in medical image analysis, Med. Image Anal. 42 (2017) 60-80.

[8] A. Hamidinekoo, E. Denton, A. Rampun, K. Honnor, R. Zwiggelaar, Deep learning in mammography and breast histology, an overview and future trends, Med Image Anal 47 (2018) 45-67.

[9] N. F. Boyd, J. W. Byng, R. A. Jong, E. K. Fishell, L. E. Little, A. B. Miller, G. A. Lockwood, D. L. Tritchler, M. J. Yaffe, Quantitative classification of mammographic densities and breast cancer risk: Results from the canadian national breast screening study, J. Nat. Cancer Inst. 87 (1995) 670-675.

[10] J. Suckling et al., The mammographic image analysis society digital mammogram database, in: Proc. Excerpta Med. Int. Congr. Ser., pp. $375-378$.

[11] A. Oliver, J. Freixenet, R. Martí, E. P. J. Pont, E. R. E. Denton, R. Zwiggelaar, A novel breast tissue density classification methodology, IEEE Trans. Inf. Technol. Biomed. 12 (2008) 55-65.

[12] K. Bovis, S. Singh, Classification of mammographic breast density using a combined classifier paradigm, in: Proc. Int. Workshop on Digital Mammography, Lecture Notes in Computer Science, Springer, 2002, pp. $177-180$.

[13] M. Muštra, M. Grgić, K. Delać, Breast density classification using multiple feature selection, Automatika 53 (2012) 362-372.

[14] N. M. Parthaláin, R. Jensen, Q. Shen, R. Zwiggelaar, Fuzzy-rough approaches for mammographic risk analysis, Intell. Data Anal. 14 (2010) $225-244$.

[15] D. Raba, J. Martí, R. Martí, M. Peracaula, Breast mammography asymmetry estimation based on fractal and texture analysis, in: Proc. Computed Aided Radiology and Surgery, Berlin, Germany, volume 1398. 
[16] H. Li, M. L. Giger, O. I. Olopade, A. Margolis, L. Lan, M. R. Chinander, Computerized texture analysis of mammographic parenchymal patterns of digitized mammograms, Acad. Radiol. 12 (2005) 863-73.

[17] A. Bosch, X. Munoz, A. Oliver, J. Martí, Modeling and classifying breast tissue density in mammograms, in: Proc IEEE Comput. Soc. Conf. Comput. Vis. Pattern. Recognit. (CVPR), volume 2, pp. 15521558 .

[18] Z. Chen, E. Denton, R. Zwiggelaar, Local feature based mammographic tissue pattern modelling and breast density classification, in: Proc. 4th International Conference on Biomedical Engineering and Informatics (BMEI), volume 1, pp. 351-355.

[19] S. Petroudi, T. Kadir, M. Brady, Automatic classification of mammographic parenchymal patterns: A statistical approach, in: Proc. IEEE Conf. Eng. Med. Biol. Soc., volume 1, pp. 798-801.

[20] A. Rampun, P. J. Morrow, B. W. Scotney, R. J. Winder, Breast density classification using local ternary patterns in mammograms, in: Proc. Image Analysis and Recognition, Springer International Publishing, Cham, 2017, pp. 463-470.

[21] A. Rampun, B. W. Scotney, P. J. Morrow, H. Wang, J. Winder, Breast density classification using local quinary patterns with various neighbourhood topologies, J. Imaging 4 (2018) 14.

[22] M. George, A. Rampun, E. Denton, R. Zwiggelaar, Mammographic ellipse modelling towards birads density classification, in: Proc. International Workshop on Breast Imaging (IWDM) 2016: Breast Imaging, pp. $423-430$.

[23] M. George, R. Zwiggelaar, Breast tissue classification using local binary pattern variants: A comparative study (in press), in: Proc. International Workshop on Breast Imaging (IWDM) 2016: Breast Imaging.

[24] T. Kooi, G. Litjens, B. van Ginnekena, A. Gubern-Méridaa, C. I. Sánchez, R. Manna, A. den Heeten, N. Karssemeijer, Large scale deep learning for computer aided detection of mammographic lesions, Med. Image Anal. 35 (2017) 303-312. 
[25] A. Rampun, K. López-Linares, P. J. Morrow, B. W. Scotney, H. Wang, I. G. Ocaña, G. Maclair, R. Zwiggelaar, M. A. González Ballester, I. Macía, Breast pectoral muscle segmentation in mammograms using a modified holistically-nested edge detection network, Medical Image Analysis 57 (2019) 1-17.

[26] A. A. Mohamed, W. A. Berg, H. Peng, Y. Luo, R. C. Jankowitz, S. Wu., A deep learning method for classifying mammographic breast density categories, Med. Phys. 45 (2017) 314-321.

[27] S. Li, J. Wei, C. Heang-Ping, M. A. Helvie, M. A. Roubidoux, Y. Lu, C. Zhou, L. M. Hadjiiski, R. K. Samala, Computer-aided assessment of breast density: comparison of supervised deep learning and featurebased statistical learning, Phys. Med. Biol. 63 (2018) 15.

[28] M. Kallenberg, K. Petersen, M. Nielsen, A. Y. Ng, P. Diao, C. Igel, C. M. Vachon, K. Holland, R. R. Winkel, N. Karssemeijer, M. Lillholm, Unsupervised deep learning applied to breast density segmentation and mammographic risk scoring, IEEE Trans. Med. Imaging. 35 (2016) 1322-1331.

[29] J. Lee, R. M. Nishikawa, Automated mammographic breast density estimation using a fully convolutional network, Med. Phys. 45 (2018) $1178-1190$.

[30] I. C. Moreira, I. Amaral, I. Domingues, A. Cardoso, M. J. Cardoso, J. S. Cardoso, Inbreast: toward a full-field digital mammographic database, Acad. Radiol. 19 (2011) 236-428.

[31] A. Rampun, P. J. Morrow, B. W. Scotney, R. J. Winder, Fully automated breast boundary and pectoral muscle segmentation in mammograms, Artif. Intell. Med. 79 (2017) 28-41.

[32] T. Ojala, M. Pietikainen, T. Maenpaar, Multiresolution gray-scale and rotation invariant texture classification with local binary patterns, IEEE Trans. Pattern Anal. Mach. Intell. 24 (2002) 971-987.

[33] X. Tan, B. Triggs, Enhanced local texture feature sets for face recognition under difficult lighting conditions, IEEE Trans. Image Process. 19 (2010) 1635-1650. 
[34] L. Nanni, A. Luminia, S. Brahnam, Local binary patterns variants as texture descriptors for medical image analysis, Artif. Intell. Med. 49 (2010) 117-125.

[35] L. Liu, L. Zhao, Y. Long, G. Kuang, P. Fieguth, Extended local binary patterns for texture classification, Image Vision Comput. 30 (2012) 86 -99 .

[36] S. Liao, A. C. S. Chung, Face recognition by using enlongated local binary patterns with average maximum distance gradient magnitude, in: Proc. Computer Vision-ACCV 2007, volume 4844, Lecture Notes in Computer Science, 2007, pp. 672-679.

[37] L. Liu, S. Lao, P. W. Fieguth, Y. Guo, X. Wang, M. Pietikäinen, Median robust extended local binary pattern for texture classification, IEEE Trans. Image Process. 25 (2016) 1368-1381.

[38] J. Yang, S. Wang, Z. Lei, Y. Zhao, S. Z. Li, Spatio-temporal lbp based moving object segmentation in compressed domain, in: Proc. IEEE Ninth International Conference on Advanced Video and Signal-Based Surveillance, pp. 252-257.

[39] Z. Guo, L. Zhang, D. Zhang, A completed modeling of local binary pattern operator for texture classification, IEEE Trans. Image Process. 19 (2010) 1657-1663.

[40] Y. Gio, G. Zhao, M. Pietikäinen, Discriminative features for feature description, Pattern Recognit. 45 (2012) 3834-3843.

[41] M. Hall, E. Frank, G. Holmes, B. Pfahringer, P. Reutemann, I. H. Witten, The weka data mining software: an update, ACM SIGKDD explorations newsletter 11 (2009) 10-18. 